\title{
Delay dynamic double integral inequalities on time scales with applications
}

\author{
Sobia Rafeeq ${ }^{1}$, Humaira Kalsoom², Sabir Hussain ${ }^{3}$, Saima Rashid ${ }^{4}$ and Yu-Ming Chu ${ }^{*}$
}

"Correspondence:

chuyuming2005@126.com

${ }^{5}$ Department of Mathematics, Huzhou University, Huzhou, China

Full list of author information is

available at the end of the article

\begin{abstract}
In the article, we present the explicit bounds for three generalized delay dynamic Gronwall-Bellman type integral inequalities on time scales, which are the unification of continuous and discrete results. As applications, the boundedness for the solutions of delay dynamic integro-differential equations with initial conditions is discussed.
\end{abstract}

MSC: 26D10; 34C11;39A12

Keywords: Delay integral inequality; Time scale; Dynamic equation; Discrete inequality; Boundedness

\section{Introduction}

Theory of time scales is the unification of both continuous and discrete analysis due to Stephen Hilger [1] in his PhD thesis, and it has wide applications in quantum calculus and difference and differential calculus. Due to its vast contributions in different branches of mathematics, it attracts the researchers and mathematicians to work on it. The role of inequalities cannot be forgot because they have huge contributions in the theory of differential equations [2-22], bivariate means [23-31], calculation and optimization [3249], special functions [50-69], probability and statistics [70-75], and so on.

It is well known that the explicit bounds for integral inequalities on unknown functions are very useful in the qualitative and quantitative analysis for the solutions of differential, integral, and integro-differential equations [76-100]. One of the most important inequalities in mathematics is the Gronwall inequality, it is an indispensable tool to obtain various estimates in the theory of ordinary and stochastic differential equations. The differential form of this inequality was proved by Gronwall [101] and its integral form was proved by Bellman [102]. Gronwall-Bellman integral inequalities have a lot of contributions to analyzing the behavior of solutions of the differential and integral equations. Recently, to dealt with the difficulties encountered in the solutions of differential and difference equations, the Gronwall-Bellman integral inequality on time scales has become one of the most important topics in mathematics research. A lot of work has been done in this area [103-106]. To discuss the abstract analysis of the solutions of certain types of dynamic equations, Gronwall-Bellman delay type inequality on time scales can play a significant role, but it still cannot deal with the abstract analysis for the solutions of some more general differential and difference equations. This gives us the motivation to discuss some

(c) The Author(s) 2020. This article is licensed under a Creative Commons Attribution 4.0 International License, which permits use, sharing, adaptation, distribution and reproduction in any medium or format, as long as you give appropriate credit to the original author(s) and the source, provide a link to the Creative Commons licence, and indicate if changes were made. The images or other third party material in this article are included in the article's Creative Commons licence, unless indicated otherwise in a credit line to the material. If material is not included in the article's Creative Commons licence and your intended use is not permitted by statutory regulation or exceeds the permitted use, you will need to obtain permission directly from the copyright holder. To view a copy of this licence, visit http://creativecommons.org/licenses/by/4.0/. 
generalized delay integral inequalities on time scales. The main purpose of the article is to provide the explicit bounds for some delay double integral inequalities on time scales, present the characteristics of the solutions of certain integro-differential equations, and establish their discrete inequalities with initial conditions by use of the analytical and numerical methods in our obtained results.

\section{Preliminaries}

The time scale $\mathbb{T}$ is a nonempty closed subset of the real numbers set $\mathbb{R}$, its forward jump operator $\sigma: \mathbb{T} \rightarrow \mathbb{T}$ for $t \in \mathbb{T}$ is defined by $\sigma(t):=\inf \{r \in \mathbb{T}: r>t\}$ and its backward jump operator $\rho: \mathbb{T} \rightarrow \mathbb{T}$ for $t \in \mathbb{T}$ is defined by $\rho(t):=\sup \{r \in \mathbb{T}: r<t\}$. The derived set $\mathbb{T}^{k}$ is defined as follows: if $\mathbb{T}$ has a left-scattered maximum $m$, then $\mathbb{T}^{k}=\mathbb{T}-\{m\}$; otherwise, $\mathbb{T}^{k}=\mathbb{T}$.

Lemma 2.1 (see [107]) Let $a, x \in \mathbb{T}^{k}$ with $x>a, f: \mathbb{T} \times \mathbb{T}^{k} \rightarrow \mathbb{R}$ be continuous at $(x, x)$, $f^{\Delta}(x, \cdot)$ be the derivative of $f$ with respect to its first variable such that it is right-dense continuous on $[a, \sigma(x)]$, and $g(x)=\int_{a}^{x} f(x, \tau) \Delta \tau$. If, for each $\epsilon>0$, there exists a neighborhood $U$ of $x$ independent of $\tau \in[a, \sigma(x)]$ such that

$$
\left|f(\sigma(x), \tau)-f(y, \tau)-f^{\Delta}(x, \tau)(\sigma(x)-y)\right| \leq \epsilon|\sigma(x)-y|
$$

for all $y \in U$, then

$$
g^{\Delta}(x)=\int_{a}^{x} f^{\Delta}(x, \tau) \Delta \tau+f(\sigma(x), x) .
$$

Theorem 2.2 (see [108]) Let $f_{1}: \mathbb{R} \rightarrow \mathbb{R}$ be continuous and differentiable and $f_{2}: \mathbb{T} \rightarrow \mathbb{R}$ be delta differentiable. Then $f_{1}^{\circ} f_{2}: \mathbb{T} \rightarrow \mathbb{R}$ is delta differentiable and

$$
\left(f_{1}^{\circ} f_{2}\right)^{\Delta}(x)=\left\{\int_{0}^{1} f_{1}^{\prime}\left(f_{2}(x)+h \mu(x) f_{2}^{\Delta}(x)\right) d h\right\} f_{2}^{\Delta}(x) .
$$

Theorem 2.3 (see [108]) Let $h_{1}: \mathbb{T} \rightarrow \mathbb{R}$ be strictly increasing such that $\overline{\mathbb{T}}=h_{1}(\mathbb{T})$ is a time scale, and $h_{2}: \overline{\mathbb{T}} \rightarrow \mathbb{R}$. Then

$$
\left(h_{2}^{\circ} h_{1}\right)^{\Delta}=\left(h_{2}^{\bar{\Delta} \circ} h_{1}\right) h_{1}^{\Delta}
$$

if $h_{1}^{\Delta}(x)$ and $h_{2}^{\bar{\Delta}}\left(h_{1}(x)\right)$ exist for $x \in \mathbb{T}^{k}$.

\section{Main results}

Throughout the section, $\mathbb{R}$ represents the set of real numbers, $\mathbb{R}_{0}^{+}=[0, \infty), \mathbb{R}_{1}^{+}=[1, \infty)$, $\mathbb{Z}$ represents the set of integers, $\mathbb{N}_{0}$ represents the set of nonnegative integers, $\mathbb{A}_{j} \subseteq \mathbb{N}_{0}$ $(1 \leq j \leq 2), x_{0 j} \in \mathbb{T}, \mathbb{T}_{j}=\left[x_{0 j}, \infty\right)_{\mathbb{T}} \subseteq \mathbb{T}^{k}$ is the time scale, $\rho_{j i}$ is the backward jump operator, $X^{\Delta y_{i}}\left(y_{1}, y_{2}, \ldots, y_{n}\right)(1 \leq i \leq n)$ is the partial delta-derivative of $X$ with respect to its $i$ th variable and $\Delta y_{i} X\left(y_{1}, y_{2}, \ldots, y_{n}\right)$ is the forward difference of $X$ with respect to its $i$ th variable.

Theorem 3.1 Let $u, r_{i}, a_{j}: \mathbb{T}_{1} \times \mathbb{T}_{2} \rightarrow \mathbb{R}_{0}^{+}$and $f_{i}, g_{i}, f_{i}^{\Delta x_{1}}: \mathbb{T}_{1}^{2} \times \mathbb{T}_{2}^{2} \rightarrow \mathbb{R}_{0}^{+}$be nonnegative and right-dense continuous such that $a_{j}$ is nondecreasing with respect to its each 
variable, let $\gamma_{j i}: \mathbb{T}_{j} \rightarrow \mathbb{R}_{0}^{+}$be nonnegative, nondecreasing, and right-dense continuous such that $\gamma_{j i}\left(x_{j}\right) \leq x_{j}$ and $\gamma_{j i}^{\Delta}\left(x_{j}\right)>0, \mu_{j i}: \mathbb{T}_{j} \rightarrow \mathbb{T}$ such that $\mu_{j i}\left(x_{j}\right) \leq x_{j}$ and $-\infty<\mathfrak{p}_{j}=$ $\inf \left\{\min \left(\mu_{j i}\left(x_{j}\right)\right), x_{j} \in \mathbb{T}_{j}\right\} \leq x_{0 j}, \mathfrak{a}:\left(\left[\mathfrak{p}_{1}, x_{01}\right] \times\left[\mathfrak{p}_{2}, x_{02}\right]\right)_{\mathbb{T}^{2}} \rightarrow \mathbb{R}_{0}^{+}$be nonnegative and rightdense continuous, $w, w_{j}: \mathbb{R}_{0}^{+} \rightarrow \mathbb{R}_{0}^{+}$be nonnegative, nondecreasing, and continuous such that $w(p)>0, w_{j}(p)>0$ for $p>0$ and

$$
\begin{aligned}
& w\left(u\left(x_{1}, x_{2}\right)\right) \\
& \leq a_{1}\left(x_{1}, x_{2}\right)+a_{2}\left(x_{1}, x_{2}\right) \sum_{i=1}^{n} \int_{\gamma_{1 i}\left(x_{01}\right)}^{\gamma_{1 i}\left(x_{1}\right)} \int_{\gamma_{2 i}\left(x_{02}\right)}^{\gamma_{2 i}\left(x_{2}\right)} w_{1}\left(u\left(\mu_{1 i}\left(t_{1}\right), \mu_{2 i}\left(t_{2}\right)\right)\right) \\
& \quad \times\left[f _ { i } ( x _ { 1 } , t _ { 1 } , x _ { 2 } , t _ { 2 } ) \left\{w_{2}\left(u\left(\mu_{1 i}\left(t_{1}\right), \mu_{2 i}\left(t_{2}\right)\right)\right)+\int_{\gamma_{1 i}\left(x_{01}\right)}^{t_{1}} \int_{\gamma_{2 i}\left(x_{02}\right)}^{t_{2}} g_{i}\left(t_{1}, m_{1}, t_{2}, m_{2}\right)\right.\right. \\
& \left.\left.\quad \times w_{2}\left(u\left(\mu_{1 i}\left(m_{1}\right), \mu_{2 i}\left(m_{2}\right)\right)\right) \Delta m_{2} \Delta m_{1}\right\}+r_{i}\left(t_{1}, t_{2}\right)\right] \Delta t_{2} \Delta t_{1}
\end{aligned}
$$

for $\left(x_{1}, x_{2}\right) \in \mathbb{T}_{1} \times \mathbb{T}_{2}$ with the initial condition

$$
\left\{\begin{array}{l}
w\left(u\left(x_{1}, x_{2}\right)\right)=\mathfrak{a}\left(x_{1}, x_{2}\right), \quad x_{1} \in\left[\mathfrak{p}_{1}, x_{01}\right]_{\mathbb{T}} \text { or } x_{2} \in\left[\mathfrak{p}_{2}, x_{02}\right]_{\mathbb{T}} \\
\mathfrak{a}\left(\mu_{1 i}\left(x_{1}\right), \mu_{2 i}\left(x_{2}\right)\right) \leq a_{1}\left(x_{1}, x_{2}\right), \quad \mu_{1 i}\left(x_{1}\right) \leq x_{01} \text { or } \mu_{2 i}\left(x_{2}\right) \leq x_{02}
\end{array}\right.
$$

Then

$$
u\left(x_{1}, x_{2}\right) \leq w^{-1}\left(G_{1}^{-1}\left(G_{2}^{-1}\left(G_{2}\left(b_{1}\left(x_{1}, x_{2}\right)\right)+a_{2}\left(x_{1}, x_{2}\right) c\left(x_{1}, x_{2}\right)\right)\right)\right)
$$

for all $x_{01} \leq x_{1} \leq \tilde{x}_{1}$ and $x_{02} \leq x_{2} \leq \tilde{x}_{2}$ if

$$
\begin{aligned}
& b_{1}\left(x_{1}, x_{2}\right)= G_{1}\left(a_{1}\left(x_{1}, x_{2}\right)\right)+a_{2}\left(x_{1}, x_{2}\right) \sum_{i=1}^{n} \int_{\gamma_{1 i}\left(x_{01}\right)}^{\gamma_{1 i}\left(x_{1}\right)} \int_{\gamma_{2 i}\left(x_{02}\right)}^{\gamma_{2 i}\left(x_{2}\right)} r_{i}\left(t_{1}, t_{2}\right) \Delta t_{2} \Delta t_{1}, \\
& \begin{aligned}
c\left(x_{1}, x_{2}\right)= & \sum_{i=1}^{n} \int_{\gamma_{1 i}\left(x_{01}\right)}^{\gamma_{1 i}\left(x_{1}\right)} \int_{\gamma_{2 i}\left(x_{02}\right)}^{\gamma_{2 i}\left(x_{2}\right)} f_{i}\left(x_{1}, t_{1}, x_{2}, t_{2}\right) \\
& \times\left(1+\int_{\gamma_{1 i}\left(x_{01}\right)}^{t_{1}} \int_{\gamma_{2 i}\left(x_{02}\right)}^{t_{2}} g_{i}\left(t_{1}, m_{1}, t_{2}, m_{2}\right) \Delta m_{2} \Delta m_{1}\right) \Delta t_{2} \Delta t_{1},
\end{aligned} \\
& G_{1}(s)=\int_{s_{1}}^{s} \frac{\Delta p}{w_{1}\left(w^{-1}(p)\right)}, \quad s>s_{1}>0 \text { with } G_{1}(\infty)=\infty, \\
& G_{2}(s)=\int_{s_{2}}^{s} \frac{\Delta p}{w_{2}\left(w^{-1}\left(G_{1}^{-1}(p)\right)\right)}, \quad s>s_{2}>0 \text { with } G_{2}(\infty)=\infty,
\end{aligned}
$$

where $G_{1}^{-1}$ and $G_{2}^{-1}$ are respectively the inverse functions of $G_{1}$ and $G_{2}$, and $\tilde{x}_{1}, \tilde{x}_{2}$ are chosen such that

$$
\begin{aligned}
& G_{2}\left(b_{1}\left(x_{1}, x_{2}\right)\right)+a_{2}\left(x_{1}, x_{2}\right) c\left(x_{1}, x_{2}\right) \in \operatorname{Dom}\left(G_{2}^{-1}\right), \\
& G_{2}^{-1}\left(G_{2}\left(b_{1}\left(x_{1}, x_{2}\right)\right)+a_{2}\left(x_{1}, x_{2}\right) c\left(x_{1}, x_{2}\right)\right) \in \operatorname{Dom}\left(G_{1}^{-1}\right), \\
& G_{1}^{-1}\left(G_{2}^{-1}\left(G_{2}\left(b_{1}\left(x_{1}, x_{2}\right)\right)+a_{2}\left(x_{1}, x_{2}\right) c\left(x_{1}, x_{2}\right)\right)\right) \in \operatorname{Dom}\left(w^{-1}\right) .
\end{aligned}
$$


Proof For fixed numbers $\bar{x}_{1} \in \mathbb{T}_{1}, \bar{x}_{2} \in \mathbb{T}_{2}$ with $x_{01} \leq \bar{x}_{1} \leq \tilde{x}_{1}$ and $x_{02} \leq \bar{x}_{2} \leq \tilde{x}_{2}$, inequality (3.1) can be rewritten as follows:

$$
\begin{aligned}
& w\left(u\left(x_{1}, x_{2}\right)\right) \\
& \leq a_{1}\left(\bar{x}_{1}, \bar{x}_{2}\right)+a_{2}\left(\bar{x}_{1}, \bar{x}_{2}\right) \sum_{i=1}^{n} \int_{\gamma_{1 i}\left(x_{01}\right)}^{\gamma_{1 i}\left(x_{1}\right)} \int_{\gamma_{2 i}\left(x_{02}\right)}^{\gamma_{2 i}\left(x_{2}\right)} w_{1}\left(u\left(\mu_{1 i}\left(t_{1}\right), \mu_{2 i}\left(t_{2}\right)\right)\right) \\
& \quad \times\left[f _ { i } ( x _ { 1 } , t _ { 1 } , x _ { 2 } , t _ { 2 } ) \left\{w_{2}\left(u\left(\mu_{1 i}\left(t_{1}\right), \mu_{2 i}\left(t_{2}\right)\right)\right)+\int_{\gamma_{1 i}\left(x_{01}\right)}^{t_{1}} \int_{\gamma_{2 i}\left(x_{02}\right)}^{t_{2}} g_{i}\left(t_{1}, m_{1}, t_{2}, m_{2}\right)\right.\right. \\
& \left.\left.\quad \times w_{2}\left(u\left(\mu_{1 i}\left(m_{1}\right), \mu_{2 i}\left(m_{2}\right)\right)\right) \Delta m_{2} \Delta m_{1}\right\}+r_{i}\left(t_{1}, t_{2}\right)\right] \Delta t_{2} \Delta t_{1} .
\end{aligned}
$$

Let

$$
\begin{aligned}
& \xi_{1}\left(x_{1}, x_{2}\right) \\
& =a_{1}\left(\bar{x}_{1}, \bar{x}_{2}\right)+a_{2}\left(\bar{x}_{1}, \bar{x}_{2}\right) \sum_{i=1}^{n} \int_{\gamma_{1 i}\left(x_{01}\right)}^{\gamma_{1 i}\left(x_{1}\right)} \int_{\gamma_{2 i}\left(x_{02}\right)}^{\gamma_{2 i}\left(x_{2}\right)} w_{1}\left(u\left(\mu_{1 i}\left(t_{1}\right), \mu_{2 i}\left(t_{2}\right)\right)\right) \\
& \quad \times\left[f _ { i } ( x _ { 1 } , t _ { 1 } , x _ { 2 } , t _ { 2 } ) \left\{w_{2}\left(u\left(\mu_{1 i}\left(t_{1}\right), \mu_{2 i}\left(t_{2}\right)\right)\right)+\int_{\gamma_{1 i}\left(x_{01}\right)}^{t_{1}} \int_{\gamma_{2 i}\left(x_{02}\right)}^{t_{2}} g_{i}\left(t_{1}, m_{1}, t_{2}, m_{2}\right)\right.\right. \\
& \left.\left.\quad \times w_{2}\left(u\left(\mu_{1 i}\left(m_{1}\right), \mu_{2 i}\left(m_{2}\right)\right)\right) \Delta m_{2} \Delta m_{1}\right\}+r_{i}\left(t_{1}, t_{2}\right)\right] \Delta t_{2} \Delta t_{1}
\end{aligned}
$$

for $x_{1} \in\left[x_{01}, \bar{x}_{1}\right]_{\mathbb{T}}$ and $x_{2} \in\left[x_{02}, \bar{x}_{2}\right]_{\mathbb{T}}$. Then

$$
\xi_{1}\left(x_{01}, x_{2}\right)=\xi_{1}\left(x_{1}, x_{02}\right)=a_{1}\left(\bar{x}_{1}, \bar{x}_{2}\right)
$$

and

$$
w\left(u\left(x_{1}, x_{2}\right)\right) \leq \xi_{1}\left(x_{1}, x_{2}\right), \quad u\left(x_{1}, x_{2}\right) \leq w^{-1}\left(\xi_{1}\left(x_{1}, x_{2}\right)\right) .
$$

If $\mu_{1 i}\left(x_{1}\right) \geq x_{01}$ and $\mu_{2 i}\left(x_{2}\right) \geq x_{02}$ for $x_{1} \in\left[x_{01}, \bar{x}_{1}\right]_{\mathbb{T}}$ and $x_{2} \in\left[x_{02}, \bar{x}_{2}\right]_{\mathbb{T}}$, then $\mu_{1 i}\left(x_{1}\right) \in$ $\left[x_{01}, \bar{x}_{1}\right]_{\mathbb{T}}, \mu_{2 i}\left(x_{1}\right) \in\left[x_{02}, \bar{x}_{2}\right]_{\mathbb{T}}$, and

$$
u\left(\mu_{1 i}\left(x_{1}\right), \mu_{2 i}\left(x_{2}\right)\right) \leq w^{-1}\left(\xi_{1}\left(\mu_{1 i}\left(x_{1}\right), \mu_{2 i}\left(x_{2}\right)\right)\right) \leq w^{-1}\left(\xi_{1}\left(x_{1}, x_{2}\right)\right) .
$$

On the other hand, if $\mu_{1 i}\left(x_{1}\right) \leq x_{01}$ or $\mu_{2 i}\left(x_{2}\right) \leq x_{02}$, then from (3.2) we have

$$
\begin{aligned}
u\left(\mu_{1 i}\left(x_{1}\right), \mu_{2 i}\left(x_{2}\right)\right) & =w^{-1}\left(\mathfrak{a}\left(\mu_{1 i}\left(x_{1}\right), \mu_{2 i}\left(x_{2}\right)\right)\right) \\
& \leq w^{-1}\left(a_{1}\left(x_{1}, x_{2}\right)\right) \leq w^{-1}\left(\xi_{1}\left(x_{1}, x_{2}\right)\right) .
\end{aligned}
$$

It follows from (3.7) and (3.8) that

$$
u\left(\mu_{1 i}\left(x_{1}\right), \mu_{2 i}\left(x_{2}\right)\right) \leq w^{-1}\left(\xi_{1}\left(x_{1}, x_{2}\right)\right)
$$

for $x_{1} \in\left[x_{01}, \bar{x}_{1}\right]_{\mathbb{T}}$ and $x_{2} \in\left[x_{02}, \bar{x}_{2}\right]_{\mathbb{T}}$. 
From Lemma 2.1, (3.4), and (3.9), one has

$$
\begin{aligned}
\xi_{1}^{\Delta x_{1}}\left(x_{1}, x_{2}\right) \\
=a_{2}\left(\bar{x}_{1}, \bar{x}_{2}\right) \sum_{i=1}^{n} \gamma_{1 i}^{\Delta}\left(x_{1}\right) \int_{\gamma_{2 i}\left(x_{02}\right)}^{\gamma_{2 i}\left(x_{2}\right)} w_{1}\left(u\left(\mu_{1 i}\left(\gamma_{1 i}\left(x_{1}\right)\right), \mu_{2 i}\left(t_{2}\right)\right)\right) \\
\times\left[f _ { i } ( \sigma ( x _ { 1 } ) , \gamma _ { 1 i } ( x _ { 1 } ) , x _ { 2 } , t _ { 2 } ) \left\{w_{2}\left(u\left(\mu_{1 i}\left(\gamma_{1 i}\left(x_{1}\right)\right), \mu_{2 i}\left(t_{2}\right)\right)\right)\right.\right. \\
+\int_{\gamma_{1 i}\left(x_{01}\right)}^{\gamma_{1 i}\left(x_{1}\right)} \int_{\gamma_{2 i}\left(x_{02}\right)}^{t_{2}} g_{i}\left(\gamma_{1 i}\left(x_{1}\right), m_{1}, t_{2}, m_{2}\right) \\
\left.\left.\quad \times w_{2}\left(u\left(\mu_{1 i}\left(m_{1}\right), \mu_{2 i}\left(m_{2}\right)\right)\right) \Delta m_{2} \Delta m_{1}\right\}+r_{i}\left(\gamma_{1 i}\left(x_{1}\right), t_{2}\right)\right] \Delta t_{2} \\
+a_{2}\left(\bar{x}_{1}, \bar{x}_{2}\right) \sum_{i=1}^{n} \int_{\gamma_{1 i}\left(x_{01}\right)}^{\gamma_{1 i}\left(x_{1}\right)}\left[\int_{\gamma_{2 i}\left(x_{02}\right)}^{\gamma_{2 i}\left(x_{2}\right)} w_{1}\left(u\left(\mu_{1 i}\left(t_{1}\right), \mu_{2 i}\left(t_{2}\right)\right)\right)\right. \\
\quad \times\left[f _ { i } ( x _ { 1 } , t _ { 1 } , x _ { 2 } , t _ { 2 } ) \left\{w_{2}\left(u\left(\mu_{1 i}\left(t_{1}\right), \mu_{2 i}\left(t_{2}\right)\right)\right)\right.\right. \\
\left.+\int_{\gamma_{1 i}\left(x_{01}\right)}^{t_{1}} \int_{\gamma_{2 i}\left(x_{02}\right)}^{t_{2}} g_{i}\left(t_{1}, m_{1}, t_{2}, m_{2}\right) w_{2}\left(u\left(\mu_{1 i}\left(m_{1}\right), \mu_{2 i}\left(m_{2}\right)\right)\right) \Delta m_{2} \Delta m_{1}\right\} \\
\left.\left.+r_{i}\left(t_{1}, t_{2}\right)\right] \Delta t_{2}\right]^{\Delta x_{1}} \Delta t_{1}
\end{aligned}
$$

and

$$
\begin{aligned}
& \xi_{1}^{\Delta x_{1}}\left(x_{1}, x_{2}\right) \\
& \leq a_{2}\left(\bar{x}_{1}, \bar{x}_{2}\right) \sum_{i=1}^{n} \gamma_{1 i}^{\Delta}\left(x_{1}\right) \int_{\gamma_{2 i}\left(x_{02}\right)}^{\gamma_{2 i}\left(x_{2}\right)} w_{1}\left(w^{-1}\left(\xi_{1}\left(\gamma_{1 i}\left(x_{1}\right), t_{2}\right)\right)\right) \\
& \times {\left[f _ { i } ( \sigma ( x _ { 1 } ) , \gamma _ { 1 i } ( x _ { 1 } ) , x _ { 2 } , t _ { 2 } ) \left\{w_{2}\left(w^{-1}\left(\xi_{1}\left(\gamma_{1 i}\left(x_{1}\right), t_{2}\right)\right)\right)\right.\right.} \\
&+\int_{\gamma_{1 i}\left(x_{01}\right)}^{\gamma_{1 i}\left(x_{1}\right)} \int_{\gamma_{2 i}\left(x_{02}\right)}^{t_{2}} g_{i}\left(\gamma_{1 i}\left(x_{1}\right), m_{1}, t_{2}, m_{2}\right) \\
&\left.\left.\times w_{2}\left(w^{-1}\left(\xi_{1}\left(m_{1}, m_{2}\right)\right)\right) \Delta m_{2} \Delta m_{1}\right\}+r_{i}\left(\gamma_{1 i}\left(x_{1}\right), t_{2}\right)\right] \Delta t_{2} \\
&+a_{2}\left(\bar{x}_{1}, \bar{x}_{2}\right) \sum_{i=1}^{n} \int_{\gamma_{1 i}\left(x_{01}\right)}^{\gamma_{1 i}\left(x_{1}\right)}\left[\int_{\gamma_{2 i}\left(x_{02}\right)}^{\gamma_{2 i}\left(x_{2}\right)} w_{1}\left(w^{-1}\left(\xi_{1}\left(t_{1}, t_{2}\right)\right)\right)\right. \\
& \times\left[f _ { i } ( x _ { 1 } , t _ { 1 } , x _ { 2 } , t _ { 2 } ) \left\{w_{2}\left(w^{-1}\left(\xi_{1}\left(t_{1}, t_{2}\right)\right)\right)\right.\right. \\
&\left.+\int_{\gamma_{1 i}\left(x_{01}\right)}^{t_{1}} \int_{\gamma_{2 i}\left(x_{02}\right)}^{t_{2}} g_{i}\left(t_{1}, m_{1}, t_{2}, m_{2}\right) w_{2}\left(w^{-1}\left(\xi_{1}\left(m_{1}, m_{2}\right)\right)\right) \Delta m_{2} \Delta m_{1}\right\} \\
&+\left.\left.r_{i}\left(t_{1}, t_{2}\right)\right] \Delta t_{2}\right]^{\Delta x_{1}} \Delta t_{1} .
\end{aligned}
$$

Using the fact that $w_{1}, w^{-1}$, and $\xi_{1}$ are nondecreasing, we have

$$
\xi_{1}^{\Delta x_{1}}\left(x_{1}, x_{2}\right)
$$




$$
\begin{aligned}
\leq & a_{2}\left(\bar{x}_{1}, \bar{x}_{2}\right) w_{1}\left(w^{-1}\left(\xi_{1}\left(x_{1}, x_{2}\right)\right)\right) \sum_{i=1}^{n} \gamma_{1 i}^{\Delta}\left(x_{1}\right) \int_{\gamma_{2 i}\left(x_{02}\right)}^{\gamma_{2 i}\left(x_{2}\right)}\left[f_{i}\left(\sigma\left(x_{1}\right), \gamma_{1 i}\left(x_{1}\right), x_{2}, t_{2}\right)\right. \\
& \times\left\{w_{2}\left(w^{-1}\left(\xi_{1}\left(\gamma_{1 i}\left(x_{1}\right), t_{2}\right)\right)\right)+\int_{\gamma_{1 i}\left(x_{01}\right)}^{\gamma_{1 i}\left(x_{1}\right)} \int_{\gamma_{2 i}\left(x_{02}\right)}^{t_{2}} g_{i}\left(\gamma_{1 i}\left(x_{1}\right), m_{1}, t_{2}, m_{2}\right)\right. \\
& \left.\left.\times w_{2}\left(w^{-1}\left(\xi_{1}\left(m_{1}, m_{2}\right)\right)\right) \Delta m_{2} \Delta m_{1}\right\}+r_{i}\left(\gamma_{1 i}\left(x_{1}\right), t_{2}\right)\right] \Delta t_{2} \\
& +a_{2}\left(\bar{x}_{1}, \bar{x}_{2}\right) w_{1}\left(w^{-1}\left(\xi_{1}\left(x_{1}, x_{2}\right)\right)\right) \sum_{i=1}^{n} \int_{\gamma_{1 i}\left(x_{01}\right)}^{\gamma_{1 i}\left(x_{1}\right)}\left[\int _ { \gamma _ { 2 i } ( x _ { 0 2 } ) } ^ { \gamma _ { 2 i } ( x _ { 2 } ) } \left[f_{i}\left(x_{1}, t_{1}, x_{2}, t_{2}\right)\right.\right. \\
& \times\left\{w_{2}\left(w^{-1}\left(\xi_{1}\left(t_{1}, t_{2}\right)\right)\right)+\int_{\gamma_{1 i}\left(x_{01}\right)}^{t_{1}} \int_{\gamma_{2 i}\left(x_{02}\right)}^{t_{2}} g_{i}\left(t_{1}, m_{1}, t_{2}, m_{2}\right)\right. \\
& \left.\left.\left.\times w_{2}\left(w^{-1}\left(\xi_{1}\left(m_{1}, m_{2}\right)\right)\right) \Delta m_{2} \Delta m_{1}\right\}+r_{i}\left(t_{1}, t_{2}\right)\right] \Delta t_{2}\right]^{\Delta x_{1}} \Delta t_{1} .
\end{aligned}
$$

Dividing both sides by $w_{1}\left(w^{-1}\left(\xi_{1}\left(x_{1}, x_{2}\right)\right)\right)$, we obtain

$$
\begin{aligned}
& \frac{\xi_{1}^{\Delta x_{1}}\left(x_{1}, x_{2}\right)}{w_{1}\left(w^{-1}\left(\xi_{1}\left(x_{1}, x_{2}\right)\right)\right)} \\
& \leq a_{2}\left(\bar{x}_{1}, \bar{x}_{2}\right) \sum_{i=1}^{n} \gamma_{1 i}^{\Delta}\left(x_{1}\right) \int_{\gamma_{2 i}\left(x_{02}\right)}^{\gamma_{2 i}\left(x_{2}\right)}\left[f_{i}\left(\sigma\left(x_{1}\right), \gamma_{1 i}\left(x_{1}\right), x_{2}, t_{2}\right)\right. \\
& \times\left\{w_{2}\left(w^{-1}\left(\xi_{1}\left(\gamma_{1 i}\left(x_{1}\right), t_{2}\right)\right)\right)+\int_{\gamma_{1 i}\left(x_{01}\right)}^{\gamma_{1 i}\left(x_{1}\right)} \int_{\gamma_{2 i}\left(x_{02}\right)}^{t_{2}} g_{i}\left(\gamma_{1 i}\left(x_{1}\right), m_{1}, t_{2}, m_{2}\right)\right. \\
& \left.\left.\times w_{2}\left(w^{-1}\left(\xi_{1}\left(m_{1}, m_{2}\right)\right)\right) \Delta m_{2} \Delta m_{1}\right\}+r_{i}\left(\gamma_{1 i}\left(x_{1}\right), t_{2}\right)\right] \Delta t_{2} \\
& +a_{2}\left(\bar{x}_{1}, \bar{x}_{2}\right) \sum_{i=1}^{n} \int_{\gamma_{1 i}\left(x_{01}\right)}^{\gamma_{1 i}\left(x_{1}\right)}\left[\int _ { \gamma _ { 2 i } ( x _ { 0 2 } ) } ^ { \gamma _ { 2 i } ( x _ { 2 } ) } \left[f_{i}\left(x_{1}, t_{1}, x_{2}, t_{2}\right)\right.\right. \\
& \times\left\{w_{2}\left(w^{-1}\left(\xi_{1}\left(t_{1}, t_{2}\right)\right)\right)+\int_{\gamma_{1 i}\left(x_{01}\right)}^{t_{1}} \int_{\gamma_{2 i}\left(x_{02}\right)}^{t_{2}} g_{i}\left(t_{1}, m_{1}, t_{2}, m_{2}\right)\right. \\
& \left.\left.\left.\times w_{2}\left(w^{-1}\left(\xi_{1}\left(m_{1}, m_{2}\right)\right)\right) \Delta m_{2} \Delta m_{1}\right\}+r_{i}\left(t_{1}, t_{2}\right)\right] \Delta t_{2}\right]^{\Delta x_{1}} \Delta t_{1} \\
& =a_{2}\left(\bar{x}_{1}, \bar{x}_{2}\right) \sum_{i=1}^{n}\left[\int _ { \gamma _ { 1 i } ( x _ { 0 1 } ) } ^ { \gamma _ { 1 i } ( x _ { 1 } ) } \int _ { \gamma _ { 2 i } ( x _ { 0 2 } ) } ^ { \gamma _ { 2 i } ( x _ { 2 } ) } \left[f_{i}\left(x_{1}, t_{1}, x_{2}, t_{2}\right)\right.\right. \\
& \times\left\{w_{2}\left(w^{-1}\left(\xi_{1}\left(t_{1}, t_{2}\right)\right)\right)+\int_{\gamma_{1 i}\left(x_{01}\right)}^{t_{1}} \int_{\gamma_{2 i}\left(x_{02}\right)}^{t_{2}} g_{i}\left(t_{1}, m_{1}, t_{2}, m_{2}\right)\right. \\
& \left.\left.\left.\times w_{2}\left(w^{-1}\left(\xi_{1}\left(m_{1}, m_{2}\right)\right)\right) \Delta m_{2} \Delta m_{1}\right\}+r_{i}\left(t_{1}, t_{2}\right)\right] \Delta t_{2} \Delta t_{1}\right]^{\Delta x_{1}} \text {. }
\end{aligned}
$$

Integrating over $\left[x_{01}, x_{1}\right]$, then using the definition of $G_{1}$ and (3.5), we get

$$
\begin{aligned}
& G_{1}\left(\xi_{1}\left(x_{1}, x_{2}\right)\right) \\
& \quad \leq G_{1}\left(a_{1}\left(\bar{x}_{1}, \bar{x}_{2}\right)\right)+a_{2}\left(\bar{x}_{1}, \bar{x}_{2}\right) \sum_{i=1}^{n} \int_{\gamma_{1 i}\left(x_{01}\right)}^{\gamma_{1 i}\left(x_{1}\right)} \int_{\gamma_{2 i}\left(x_{02}\right)}^{\gamma_{2 i}\left(x_{2}\right)} r_{i}\left(t_{1}, t_{2}\right) \Delta t_{2} \Delta t_{1}
\end{aligned}
$$




$$
\begin{aligned}
& +a_{2}\left(\bar{x}_{1}, \bar{x}_{2}\right) \sum_{i=1}^{n} \int_{\gamma_{1 i}\left(x_{01}\right)}^{\gamma_{1 i}\left(x_{1}\right)} \int_{\gamma_{2 i}\left(x_{02}\right)}^{\gamma_{2 i}\left(x_{2}\right)} f_{i}\left(x_{1}, t_{1}, x_{2}, t_{2}\right) \\
& \times\left\{w_{2}\left(w^{-1}\left(\xi_{1}\left(t_{1}, t_{2}\right)\right)\right)+\int_{\gamma_{1 i}\left(x_{01}\right)}^{t_{1}} \int_{\gamma_{2 i}\left(x_{02}\right)}^{t_{2}} g_{i}\left(t_{1}, m_{1}, t_{2}, m_{2}\right)\right. \\
& \left.\times w_{2}\left(w^{-1}\left(\xi_{1}\left(m_{1}, m_{2}\right)\right)\right) \Delta m_{2} \Delta m_{1}\right\} \Delta t_{2} \Delta t_{1} \\
& \leq G_{1}\left(a_{1}\left(\bar{x}_{1}, \bar{x}_{2}\right)\right)+a_{2}\left(\bar{x}_{1}, \bar{x}_{2}\right) \sum_{i=1}^{n} \int_{\gamma_{1 i}\left(x_{01}\right)}^{\gamma_{1 i}\left(\bar{x}_{1}\right)} \int_{\gamma_{2 i}\left(x_{02}\right)}^{\gamma_{2 i}\left(\bar{x}_{2}\right)} r_{i}\left(t_{1}, t_{2}\right) \Delta t_{2} \Delta t_{1} \\
& +a_{2}\left(\bar{x}_{1}, \bar{x}_{2}\right) \sum_{i=1}^{n} \int_{\gamma_{1 i}\left(x_{01}\right)}^{\gamma_{1 i}\left(x_{1}\right)} \int_{\gamma_{2 i}\left(x_{02}\right)}^{\gamma_{2 i}\left(x_{2}\right)} f_{i}\left(x_{1}, t_{1}, x_{2}, t_{2}\right) \\
& \times\left\{w_{2}\left(w^{-1}\left(\xi_{1}\left(t_{1}, t_{2}\right)\right)\right)+\int_{\gamma_{1 i}\left(x_{01}\right)}^{t_{1}} \int_{\gamma_{2 i}\left(x_{02}\right)}^{t_{2}} g_{i}\left(t_{1}, m_{1}, t_{2}, m_{2}\right)\right. \\
& \left.\times w_{2}\left(w^{-1}\left(\xi_{1}\left(m_{1}, m_{2}\right)\right)\right) \Delta m_{2} \Delta m_{1}\right\} \Delta t_{2} \Delta t_{1} \\
& =b_{1}\left(\bar{x}_{1}, \bar{x}_{2}\right)+a_{2}\left(\bar{x}_{1}, \bar{x}_{2}\right) \sum_{i=1}^{n} \int_{\gamma_{1 i}\left(x_{01}\right)}^{\gamma_{1 i}\left(x_{1}\right)} \int_{\gamma_{2 i}\left(x_{02}\right)}^{\gamma_{2 i}\left(x_{2}\right)} f_{i}\left(x_{1}, t_{1}, x_{2}, t_{2}\right) \\
& \times\left\{w_{2}\left(w^{-1}\left(\xi_{1}\left(t_{1}, t_{2}\right)\right)\right)+\int_{\gamma_{1 i}\left(x_{01}\right)}^{t_{1}} \int_{\gamma_{2 i}\left(x_{02}\right)}^{t_{2}} g_{i}\left(t_{1}, m_{1}, t_{2}, m_{2}\right)\right. \\
& \left.\times w_{2}\left(w^{-1}\left(\xi_{1}\left(m_{1}, m_{2}\right)\right)\right) \Delta m_{2} \Delta m_{1}\right\} \Delta t_{2} \Delta t_{1} \text {. }
\end{aligned}
$$

Let

$$
\begin{aligned}
\zeta_{1}\left(x_{1}, x_{2}\right)= & b_{1}\left(\bar{x}_{1}, \bar{x}_{2}\right)+a_{2}\left(\bar{x}_{1}, \bar{x}_{2}\right) \sum_{i=1}^{n} \int_{\gamma_{1 i}\left(x_{01}\right)}^{\gamma_{1 i}\left(x_{1}\right)} \int_{\gamma_{2 i}\left(x_{02}\right)}^{\gamma_{2 i}\left(x_{2}\right)} f_{i}\left(x_{1}, t_{1}, x_{2}, t_{2}\right) \\
& \times\left\{w_{2}\left(w^{-1}\left(\xi_{1}\left(t_{1}, t_{2}\right)\right)\right)+\int_{\gamma_{1 i}\left(x_{01}\right)}^{t_{1}} \int_{\gamma_{2 i}\left(x_{02}\right)}^{t_{2}} g_{i}\left(t_{1}, m_{1}, t_{2}, m_{2}\right)\right. \\
& \left.\times w_{2}\left(w^{-1}\left(\xi_{1}\left(m_{1}, m_{2}\right)\right)\right) \Delta m_{2} \Delta m_{1}\right\} \Delta t_{2} \Delta t_{1} .
\end{aligned}
$$

Then we have

$$
\zeta_{1}\left(x_{01}, x_{2}\right)=\zeta_{1}\left(x_{1}, x_{02}\right)=b_{1}\left(\bar{x}_{1}, \bar{x}_{2}\right)
$$

and

$$
\xi_{1}\left(x_{1}, x_{2}\right) \leq G_{1}^{-1}\left(\zeta_{1}\left(x_{1}, x_{2}\right)\right)
$$

It follows from Lemma 2.1 and (3.11) that

$$
\begin{aligned}
\zeta_{1}^{\Delta x_{1}}\left(x_{1}, x_{2}\right)= & a_{2}\left(\bar{x}_{1}, \bar{x}_{2}\right) \sum_{i=1}^{n} \gamma_{1 i}^{\Delta}\left(x_{1}\right) \int_{\gamma_{2 i}\left(x_{02}\right)}^{\gamma_{2 i}\left(x_{2}\right)} f_{i}\left(\sigma\left(x_{1}\right), \gamma_{1 i}\left(x_{1}\right), x_{2}, t_{2}\right) \\
& \times\left\{w_{2}\left(w^{-1}\left(\xi_{1}\left(\gamma_{1 i}\left(x_{1}\right), t_{2}\right)\right)\right)+\int_{\gamma_{1 i}\left(x_{01}\right)}^{\gamma_{1 i}\left(x_{1}\right)} \int_{\gamma_{2 i}\left(x_{02}\right)}^{t_{2}} g_{i}\left(\gamma_{1 i}\left(x_{1}\right), m_{1}, t_{2}, m_{2}\right)\right.
\end{aligned}
$$




$$
\begin{aligned}
& \left.\times w_{2}\left(w^{-1}\left(\xi_{1}\left(m_{1}, m_{2}\right)\right)\right) \Delta m_{2} \Delta m_{1}\right\} \Delta t_{2} \\
& +a_{2}\left(\bar{x}_{1}, \bar{x}_{2}\right) \sum_{i=1}^{n} \int_{\gamma_{1 i}\left(x_{01}\right)}^{\gamma_{1 i}\left(x_{1}\right)}\left[\int_{\gamma_{2 i}\left(x_{02}\right)}^{\gamma_{2 i}\left(x_{2}\right)} f_{i}\left(x_{1}, t_{1}, x_{2}, t_{2}\right)\right. \\
& \times\left\{w_{2}\left(w^{-1}\left(\xi_{1}\left(t_{1}, t_{2}\right)\right)\right)+\int_{\gamma_{1 i}\left(x_{01}\right)}^{t_{1}} \int_{\gamma_{2 i}\left(x_{02}\right)}^{t_{2}} g_{i}\left(t_{1}, m_{1}, t_{2}, m_{2}\right)\right. \\
& \left.\left.\times w_{2}\left(w^{-1}\left(\xi_{1}\left(m_{1}, m_{2}\right)\right)\right) \Delta m_{2} \Delta m_{1}\right\} \Delta t_{2}\right]^{\Delta x_{1}} \Delta t_{1} .
\end{aligned}
$$

Making use of (3.13) and the fact that $w_{2}, w^{-1}, G_{1}^{-1}$, and $\zeta_{1}$ are nondecreasing, we get

$$
\begin{aligned}
\frac{\zeta_{1}{ }^{\Delta x_{1}}\left(x_{1}, x_{2}\right)}{w_{2}\left(w^{-1}\left(G_{1}^{-1}\left(\zeta_{1}\left(x_{1}, x_{2}\right)\right)\right)\right)} & a_{2}\left(\bar{x}_{1}, \bar{x}_{2}\right) \sum_{i=1}^{n}\left[\gamma_{1 i}^{\Delta}\left(x_{1}\right) \int_{\gamma_{2 i}\left(x_{02}\right)}^{\gamma_{2 i}\left(x_{2}\right)} f_{i}\left(\sigma\left(x_{1}\right), \gamma_{1 i}\left(x_{1}\right), x_{2}, t_{2}\right)\right. \\
& \times\left\{1+\int_{\gamma_{1 i}\left(x_{01}\right)}^{\gamma_{1 i}\left(x_{1}\right)} \int_{\gamma_{2 i}\left(x_{02}\right)}^{t_{2}} g_{i}\left(\gamma_{1 i}\left(x_{1}\right), m_{1}, t_{2}, m_{2}\right) \Delta m_{2} \Delta m_{1}\right\} \Delta t_{2} \\
& +\int_{\gamma_{1 i}\left(x_{01}\right)}^{\gamma_{1 i}\left(x_{1}\right)}\left[\int_{\gamma_{2 i}\left(x_{02}\right)}^{\gamma_{2 i}\left(x_{2}\right)} f_{i}\left(x_{1}, t_{1}, x_{2}, t_{2}\right)\right. \\
& \left.\left.\times\left\{1+\int_{\gamma_{1 i}\left(x_{01}\right)}^{t_{1}} \int_{\gamma_{2 i}\left(x_{02}\right)}^{t_{2}} g_{i}\left(t_{1}, m_{1}, t_{2}, m_{2}\right) \Delta m_{2} \Delta m_{1}\right\} \Delta t_{2}\right]^{\Delta x_{1}} \Delta t_{1}\right] \\
= & a_{2}\left(\bar{x}_{1}, \bar{x}_{2}\right) \sum_{i=1}^{n}\left[\int_{\gamma_{1 i}\left(x_{01}\right)}^{\gamma_{1 i}\left(x_{1}\right)} \int_{\gamma_{2 i}\left(x_{02}\right)}^{\gamma_{2 i}\left(x_{2}\right)} f_{i}\left(x_{1}, t_{1}, x_{2}, t_{2}\right)\right. \\
& \left.\times\left\{1+\int_{\gamma_{1 i}\left(x_{01}\right)}^{t_{1}} \int_{\gamma_{2 i}\left(x_{02}\right)}^{t_{2}} g_{i}\left(t_{1}, m_{1}, t_{2}, m_{2}\right) \Delta m_{2} \Delta m_{1}\right\} \Delta t_{2} \Delta t_{1}\right]^{\Delta x_{1}} .
\end{aligned}
$$

Integrating over $\left[x_{01}, x_{1}\right]$, then using the definition of $G_{2}$ and (3.12), we get

$$
\begin{aligned}
G_{2}\left(\zeta_{1}\left(x_{1}, x_{2}\right)\right) \leq & G_{2}\left(b_{1}\left(\bar{x}_{1}, \bar{x}_{2}\right)\right)+a_{2}\left(\bar{x}_{1}, \bar{x}_{2}\right) \sum_{i=1}^{n} \int_{\gamma_{1 i}\left(x_{01}\right)}^{\gamma_{1 i}\left(x_{1}\right)} \int_{\gamma_{2 i}\left(x_{02}\right)}^{\gamma_{2 i}\left(x_{2}\right)} f_{i}\left(x_{1}, t_{1}, x_{2}, t_{2}\right) \\
& \times\left\{1+\int_{\gamma_{1 i}\left(x_{01}\right)}^{t_{1}} \int_{\gamma_{2 i}\left(x_{02}\right)}^{t_{2}} g_{i}\left(t_{1}, m_{1}, t_{2}, m_{2}\right) \Delta m_{2} \Delta m_{1}\right\} \Delta t_{2} \Delta t_{1} \\
= & G_{2}\left(b_{1}\left(\bar{x}_{1}, \bar{x}_{2}\right)\right)+a_{2}\left(\bar{x}_{1}, \bar{x}_{2}\right) c\left(x_{1}, x_{2}\right) .
\end{aligned}
$$

A combination of (3.6), (3.13), and (3.15) gives the desired result (3.3).

Remark 3.2 Let $\mathbb{T}=\mathbb{Z}, a_{1}\left(x_{1}, x_{2}\right)=c, a_{2}\left(x_{1}, x_{2}\right)=w_{2}=1, \gamma_{j i}=\mu_{j i}=I, n=1, f_{i}\left(x_{1}, t_{1}, x_{2}, t_{2}\right)=$ $f\left(t_{1}, t_{2}\right), g_{i}=r_{i}=0$. Then Theorem 3.1 coincides with Theorem 2.1 in [109]. Moreover, if $w(u)=u^{p}$, then it coincides with Theorem 2.1 in [110].

Remark 3.3 Let $\mathbb{T}=\mathbb{R}, a_{1}\left(x_{1}, x_{2}\right)=c, a_{2}\left(x_{1}, x_{2}\right)=1, w_{1}=\mu_{j i}=I, f_{i}\left(x_{1}, t_{1}, x_{2}, t_{2}\right)=f_{i}\left(t_{1}, t_{2}\right)$, $g_{i}=0(1 \leq i \leq n)$. Then Theorem 3.1 leads to Theorem 2.3 in [111]. Moreover, if $r_{i}=0$, then it reduces to Theorem 2.2 in [111]. 
Remark 3.4 Let $\mathbb{T}=\mathbb{R}, w_{1}(u)=u^{q}, \mu_{j i}=I, f_{i}\left(x_{1}, t_{1}, x_{2}, t_{2}\right)=f_{i}\left(t_{1}, t_{2}\right), g_{i}=0(1 \leq i \leq n)$. Then Theorem 3.1 becomes Theorem 2.1 in [112].

Remark 3.5 Let $n=1, \gamma_{j i}=I, w_{2}=1, f_{i}\left(x_{1}, t_{1}, x_{2}, t_{2}\right)=f\left(t_{1}, t_{2}\right)$, and $g_{i}=r_{i}=0$. Theorem 3.1 coincides with Theorem 1 in [113].

Remark 3.6 Let $\mathbb{T}=\mathbb{Z}, w_{2}=1, \gamma_{j i}=\mu_{j i}=I, n=1, f_{i}\left(x_{1}, t_{1}, x_{2}, t_{2}\right)=f\left(t_{1}, t_{2}\right)$, and $g_{i}=r_{i}=0$. Then Theorem 3.1 leads to Theorem 1 in [114].

Remark 3.7 Let $\mathbb{T}=\mathbb{R}, \mu_{j i}=I, f_{i}\left(x_{1}, t_{1}, x_{2}, t_{2}\right)=f_{i}\left(t_{1}, t_{2}\right)$, and $g_{i}=0$. Then Theorem 3.1 reduces to Theorem 1 in [115].

Theorem 3.8 Let $a_{j}, r_{i}, w, w_{j}, f_{i}, g_{i}, \gamma_{j i}, \mu_{j i}$, and $\mathfrak{a}$ be defined as in Theorem 3.1 such that $w_{j+2}$ has the same conditions of $w_{j}$, and let $u: \mathbb{T}_{1} \times \mathbb{T}_{2} \rightarrow \mathbb{R}_{1}^{+}$be a right-dense continuous function such that

$$
\begin{aligned}
& w\left(u\left(x_{1}, x_{2}\right)\right) \\
& \leq a_{1}\left(x_{1}, x_{2}\right)+a_{2}\left(x_{1}, x_{2}\right) \sum_{i=1}^{n} \int_{\gamma_{1 i}\left(x_{01}\right)}^{\gamma_{1 i}\left(x_{1}\right)} \int_{\gamma_{2 i}\left(x_{02}\right)}^{\gamma_{2 i}\left(x_{2}\right)} w_{1}\left(u\left(\mu_{1 i}\left(t_{1}\right), \mu_{2 i}\left(t_{2}\right)\right)\right) \\
& \quad \times\left[f _ { i } ( x _ { 1 } , t _ { 1 } , x _ { 2 } , t _ { 2 } ) w _ { 2 } ( u ( \mu _ { 1 i } ( t _ { 1 } ) , \mu _ { 2 i } ( t _ { 2 } ) ) ) \left\{w_{3}\left(u\left(\mu_{1 i}\left(t_{1}\right), \mu_{2 i}\left(t_{2}\right)\right)\right)\right.\right. \\
& \left.\quad+\int_{\gamma_{1 i}\left(x_{01}\right)}^{t_{1}} \int_{\gamma_{2 i}\left(x_{02}\right)}^{t_{2}} g_{i}\left(t_{1}, m_{1}, t_{2}, m_{2}\right) \times w_{3}\left(u\left(\mu_{1 i}\left(m_{1}\right), \mu_{2 i}\left(m_{2}\right)\right)\right) \Delta m_{2} \Delta m_{1}\right\} \\
& \left.\quad+r_{i}\left(t_{1}, t_{2}\right) w_{4}\left(\log \left(u\left(\mu_{1 i}\left(t_{1}\right), \mu_{2 i}\left(t_{2}\right)\right)\right)\right)\right] \Delta t_{2} \Delta t_{1}
\end{aligned}
$$

for $\left(x_{1}, x_{2}\right) \in \mathbb{T}_{1} \times \mathbb{T}_{2}$ with initial condition (3.2). Then the following statements are true:

(1) If $w_{2}(u) \geq w_{4}(\log (u))$, then

$$
u\left(x_{1}, x_{2}\right) \leq w^{-1}\left(G_{1}^{-1}\left(G_{2}^{-1}\left(Q_{1}^{-1}\left(Q_{1}\left(d_{1}\left(x_{1}, x_{2}\right)\right)+a_{2}\left(x_{1}, x_{2}\right) c\left(x_{1}, x_{2}\right)\right)\right)\right)\right)
$$

for all $x_{01} \leq x_{1} \leq \tilde{x}_{1}$ and $x_{02} \leq x_{2} \leq \tilde{x}_{2}$;

(2) If $w_{2}(u)<w_{4}(\log (u))$, then

$$
u\left(x_{1}, x_{2}\right) \leq w^{-1}\left(G_{1}^{-1}\left(G_{3}^{-1}\left(Q_{2}^{-1}\left(Q_{2}\left(d_{2}\left(x_{1}, x_{2}\right)\right)+a_{2}\left(x_{1}, x_{2}\right) c\left(x_{1}, x_{2}\right)\right)\right)\right)\right)
$$

for all $x_{01} \leq x_{1} \leq \tilde{x}_{3}$ and $x_{02} \leq x_{2} \leq \tilde{x}_{4}$, where

$$
\begin{aligned}
& d_{j}\left(x_{1}, x_{2}\right)=G_{j+1}\left(G_{1}\left(a_{1}\left(x_{1}, x_{2}\right)\right)\right)+a_{2}\left(x_{1}, x_{2}\right) \sum_{i=1}^{n} \int_{\gamma_{1 i}\left(x_{01}\right)}^{\gamma_{1 i}\left(x_{1}\right)} \int_{\gamma_{2 i}\left(x_{02}\right)}^{\gamma_{2 i}\left(x_{2}\right)} r_{i}\left(t_{1}, t_{2}\right) \Delta t_{2} \Delta t_{1}, \\
& G_{3}(s)=\int_{s_{3}}^{s} \frac{\Delta p}{w_{4}\left(w^{-1}\left(G_{1}^{-1}(p)\right)\right)}, \quad s>s_{3}>0 \text { with } G_{3}(\infty)=\infty, \\
& Q_{j}(s)=\int_{s_{3+j}}^{s} \frac{\Delta p}{w_{3}\left(w^{-1}\left(G_{1}^{-1}\left(G_{j+1}^{-1}(p)\right)\right)\right)}, \quad s>s_{3+j}>0 \text { with } Q_{j}(\infty)=\infty,
\end{aligned}
$$


$c, G_{1}$, and $G_{2}$ are defined as in Theorem $3.1, G_{1}^{-1}, G_{2}^{-1}, G_{3}^{-1}$, and $G_{4}^{-1}$ are respectively the inverse functions of $G_{1}, G_{2}, G_{3}$, and $G_{4}$, and $\tilde{x}_{1}, \tilde{x}_{2}, \tilde{x}_{3}$, and $\tilde{x}_{4}$ are chosen such that

$$
\begin{aligned}
& Q_{j}\left(d_{j}\left(x_{1}, x_{2}\right)\right)+a_{2}\left(x_{1}, x_{2}\right) c\left(x_{1}, x_{2}\right) \in \operatorname{Dom}\left(Q_{j}^{-1}\right), \\
& Q_{j}^{-1}\left(Q_{j}\left(d_{j}\left(x_{1}, x_{2}\right)\right)+a_{2}\left(x_{1}, x_{2}\right) c\left(x_{1}, x_{2}\right)\right) \in \operatorname{Dom}\left(G_{j+1}^{-1}\right) \\
& G_{j+1}^{-1}\left(Q_{j}^{-1}\left(Q_{j}\left(d_{j}\left(x_{1}, x_{2}\right)\right)+a_{2}\left(x_{1}, x_{2}\right) c\left(x_{1}, x_{2}\right)\right)\right) \in \operatorname{Dom}\left(G_{1}^{-1}\right) \\
& G_{1}^{-1}\left(G_{j+1}^{-1}\left(Q_{j}^{-1}\left(Q_{j}\left(d_{j}\left(x_{1}, x_{2}\right)\right)+a_{2}\left(x_{1}, x_{2}\right) c\left(x_{1}, x_{2}\right)\right)\right)\right) \in \operatorname{Dom}\left(w^{-1}\right) .
\end{aligned}
$$

Proof Let $\bar{x}_{1} \in \mathbb{T}_{1}$ and $\bar{x}_{2} \in \mathbb{T}_{2}$ with $x_{01} \leq \bar{x}_{1} \leq \tilde{x}_{1}$ and $x_{02} \leq \bar{x}_{2} \leq \tilde{x}_{2}$. Then inequality (3.16) can be rewritten as

$$
\begin{aligned}
& w\left(u\left(x_{1}, x_{2}\right)\right) \\
& \leq a_{1}\left(\bar{x}_{1}, \bar{x}_{2}\right)+a_{2}\left(\bar{x}_{1}, \bar{x}_{2}\right) \sum_{i=1}^{n} \int_{\gamma_{1 i}\left(x_{01}\right)}^{\gamma_{1 i}\left(x_{1}\right)} \int_{\gamma_{2 i}\left(x_{02}\right)}^{\gamma_{2 i}\left(x_{2}\right)} w_{1}\left(u\left(\mu_{1 i}\left(t_{1}\right), \mu_{2 i}\left(t_{2}\right)\right)\right) \\
& \quad \times\left[f _ { i } ( x _ { 1 } , t _ { 1 } , x _ { 2 } , t _ { 2 } ) w _ { 2 } ( u ( \mu _ { 1 i } ( t _ { 1 } ) , \mu _ { 2 i } ( t _ { 2 } ) ) ) \left\{w_{3}\left(u\left(\mu_{1 i}\left(t_{1}\right), \mu_{2 i}\left(t_{2}\right)\right)\right)\right.\right. \\
& \left.\quad+\int_{\gamma_{1 i}\left(x_{01}\right)}^{t_{1}} \int_{\gamma_{2 i}\left(x_{02}\right)}^{t_{2}} g_{i}\left(t_{1}, m_{1}, t_{2}, m_{2}\right) \times w_{3}\left(u\left(\mu_{1 i}\left(m_{1}\right), \mu_{2 i}\left(m_{2}\right)\right)\right) \Delta m_{2} \Delta m_{1}\right\} \\
& \left.\quad+r_{i}\left(t_{1}, t_{2}\right) w_{4}\left(\log \left(u\left(\mu_{1 i}\left(t_{1}\right), \mu_{2 i}\left(t_{2}\right)\right)\right)\right)\right] \Delta t_{2} \Delta t_{1}
\end{aligned}
$$

for $x_{01} \leq x_{1} \leq \bar{x}_{1}$ and $x_{02} \leq x_{2} \leq \bar{x}_{2}$.

Let

$$
\begin{aligned}
& \xi_{2}\left(x_{1}, x_{2}\right) \\
& =a_{1}\left(\bar{x}_{1}, \bar{x}_{2}\right)+a_{2}\left(\bar{x}_{1}, \bar{x}_{2}\right) \sum_{i=1}^{n} \int_{\gamma_{1 i}\left(x_{01}\right)}^{\gamma_{1 i}\left(x_{1}\right)} \int_{\gamma_{2 i}\left(x_{02}\right)}^{\gamma_{2 i}\left(x_{2}\right)} w_{1}\left(u\left(\mu_{1 i}\left(t_{1}\right), \mu_{2 i}\left(t_{2}\right)\right)\right) \\
& \quad \times\left[f _ { i } ( x _ { 1 } , t _ { 1 } , x _ { 2 } , t _ { 2 } ) w _ { 2 } ( u ( \mu _ { 1 i } ( t _ { 1 } ) , \mu _ { 2 i } ( t _ { 2 } ) ) ) \left\{w_{3}\left(u\left(\mu_{1 i}\left(t_{1}\right), \mu_{2 i}\left(t_{2}\right)\right)\right)\right.\right. \\
& \left.\quad+\int_{\gamma_{1 i}\left(x_{01}\right)}^{t_{1}} \int_{\gamma_{2 i}\left(x_{02}\right)}^{t_{2}} g_{i}\left(t_{1}, m_{1}, t_{2}, m_{2}\right) \times w_{3}\left(u\left(\mu_{1 i}\left(m_{1}\right), \mu_{2 i}\left(m_{2}\right)\right)\right) \Delta m_{2} \Delta m_{1}\right\} \\
& \left.\quad+r_{i}\left(t_{1}, t_{2}\right) w_{4}\left(\log \left(u\left(\mu_{1 i}\left(t_{1}\right), \mu_{2 i}\left(t_{2}\right)\right)\right)\right)\right] \Delta t_{2} \Delta t_{1} .
\end{aligned}
$$

Then one has

$$
\xi_{2}\left(x_{01}, x_{2}\right)=\xi_{2}\left(x_{1}, x_{02}\right)=a_{1}\left(\bar{x}_{1}, \bar{x}_{2}\right)
$$

and

$$
u\left(x_{1}, x_{2}\right) \leq w^{-1}\left(\xi_{2}\left(x_{1}, x_{2}\right)\right) .
$$


On taking the identical steps as in (3.7)-(3.10), we get

$$
\begin{aligned}
& G_{1}\left(\xi_{2}\left(x_{1}, x_{2}\right)\right) \\
& \leq G_{1}\left(a_{1}\left(\bar{x}_{1}, \bar{x}_{2}\right)\right)+a_{2}\left(\bar{x}_{1}, \bar{x}_{2}\right) \sum_{i=1}^{n} \int_{\gamma_{1 i}\left(x_{01}\right)}^{\gamma_{1 i}\left(x_{1}\right)} \int_{\gamma_{2 i}\left(x_{02}\right)}^{\gamma_{2 i}\left(x_{2}\right)}\left[f_{i}\left(x_{1}, t_{1}, x_{2}, t_{2}\right)\right. \\
& \quad \times w_{2}\left(w^{-1}\left(\xi_{2}\left(t_{1}, t_{2}\right)\right)\right)\left\{w_{3}\left(w^{-1}\left(\xi_{2}\left(t_{1}, t_{2}\right)\right)\right)+\int_{\gamma_{1 i}\left(x_{01}\right)}^{t_{1}} \int_{\gamma_{2 i}\left(x_{02}\right)}^{t_{2}} g_{i}\left(t_{1}, m_{1}, t_{2}, m_{2}\right)\right. \\
& \left.\quad \times w_{3}\left(w^{-1}\left(\xi_{2}\left(m_{1}, m_{2}\right)\right)\right) \Delta m_{2} \Delta m_{1}\right\} \\
& \left.\quad+r_{i}\left(t_{1}, t_{2}\right) w_{4}\left(\log \left(w^{-1}\left(\xi_{2}\left(t_{1}, t_{2}\right)\right)\right)\right)\right] \Delta t_{2} \Delta t_{1} .
\end{aligned}
$$

For $w_{2}(\mathfrak{u}) \geq w_{4}(\log (\mathfrak{u}))$, inequality $(3.20)$ can be rewritten as follows:

$$
\begin{aligned}
& G_{1}\left(\xi_{2}\left(x_{1}, x_{2}\right)\right) \\
& \leq G_{1}\left(a_{1}\left(\bar{x}_{1}, \bar{x}_{2}\right)\right)+a_{2}\left(\bar{x}_{1}, \bar{x}_{2}\right) \sum_{i=1}^{n} \int_{\gamma_{1 i}\left(x_{01}\right)}^{\gamma_{1 i}\left(x_{1}\right)} \int_{\gamma_{2 i}\left(x_{02}\right)}^{\gamma_{2 i}\left(x_{2}\right)} w_{2}\left(w^{-1}\left(\xi_{2}\left(t_{1}, t_{2}\right)\right)\right) \\
& \quad \times\left[f _ { i } ( x _ { 1 } , t _ { 1 } , x _ { 2 } , t _ { 2 } ) \left\{w_{3}\left(w^{-1}\left(\xi_{2}\left(t_{1}, t_{2}\right)\right)\right)+\int_{\gamma_{1 i}\left(x_{01}\right)}^{t_{1}} \int_{\gamma_{2 i}\left(x_{02}\right)}^{t_{2}} g_{i}\left(t_{1}, m_{1}, t_{2}, m_{2}\right)\right.\right. \\
& \left.\left.\quad \times w_{3}\left(w^{-1}\left(\xi_{2}\left(m_{1}, m_{2}\right)\right)\right) \Delta m_{2} \Delta m_{1}\right\}+r_{i}\left(t_{1}, t_{2}\right)\right] \Delta t_{2} \Delta t_{1} .
\end{aligned}
$$

Let

$$
\begin{aligned}
\zeta_{2}\left(x_{1}, x_{2}\right)= & G_{1}\left(a_{1}\left(\bar{x}_{1}, \bar{x}_{2}\right)\right)+a_{2}\left(\bar{x}_{1}, \bar{x}_{2}\right) \sum_{i=1}^{n} \int_{\gamma_{1 i}\left(x_{01}\right)}^{\gamma_{1 i}\left(x_{1}\right)} \int_{\gamma_{2 i}\left(x_{02}\right)}^{\gamma_{2 i}\left(x_{2}\right)} w_{2}\left(w^{-1}\left(\xi_{2}\left(t_{1}, t_{2}\right)\right)\right) \\
& \times\left[f _ { i } ( x _ { 1 } , t _ { 1 } , x _ { 2 } , t _ { 2 } ) \left\{w_{3}\left(w^{-1}\left(\xi_{2}\left(t_{1}, t_{2}\right)\right)\right)+\int_{\gamma_{1 i}\left(x_{01}\right)}^{t_{1}} \int_{\gamma_{2 i}\left(x_{02}\right)}^{t_{2}} g_{i}\left(t_{1}, m_{1}, t_{2}, m_{2}\right)\right.\right. \\
& \left.\left.\times w_{3}\left(w^{-1}\left(\xi_{2}\left(m_{1}, m_{2}\right)\right)\right) \Delta m_{2} \Delta m_{1}\right\}+r_{i}\left(t_{1}, t_{2}\right)\right] \Delta t_{2} \Delta t_{1} .
\end{aligned}
$$

Then

$$
\zeta_{2}\left(x_{01}, x_{2}\right)=\zeta_{2}\left(x_{1}, x_{02}\right)=G_{1}\left(a_{1}\left(\bar{x}_{1}, \bar{x}_{2}\right)\right)
$$

and

$$
\xi_{2}\left(x_{1}, x_{2}\right) \leq G_{1}^{-1}\left(\zeta_{2}\left(x_{1}, x_{2}\right)\right)
$$

On taking the identical steps as those in (3.13)-(3.14), one has

$$
\begin{aligned}
& G_{2}\left(\zeta_{2}\left(x_{1}, x_{2}\right)\right) \\
& \leq G_{2}\left(G_{1}\left(a_{1}\left(\bar{x}_{1}, \bar{x}_{2}\right)\right)\right)+a_{2}\left(\bar{x}_{1}, \bar{x}_{2}\right) \sum_{i=1}^{n} \int_{\gamma_{1 i}\left(x_{01}\right)}^{\gamma_{1 i}\left(x_{1}\right)} \int_{\gamma_{2 i}\left(x_{02}\right)}^{\gamma_{2 i}\left(x_{2}\right)}\left[f_{i}\left(x_{1}, t_{1}, x_{2}, t_{2}\right)\right. \\
& \quad \times w_{3}\left(w^{-1}\left(G_{1}^{-1}\left(\zeta_{2}\left(t_{1}, t_{2}\right)\right)\right)\right)
\end{aligned}
$$




$$
\begin{aligned}
& \left.\times\left\{1+\int_{\gamma_{1 i}\left(x_{01}\right)}^{t_{1}} \int_{\gamma_{2 i}\left(x_{02}\right)}^{t_{2}} g_{i}\left(t_{1}, m_{1}, t_{2}, m_{2}\right) \Delta m_{2} \Delta m_{1}\right\}+r_{i}\left(t_{1}, t_{2}\right)\right] \Delta t_{2} \Delta t_{1} \\
\leq & d_{1}\left(\bar{x}_{1}, \bar{x}_{2}\right)+a_{2}\left(\bar{x}_{1}, \bar{x}_{2}\right) \sum_{i=1}^{n} \int_{\gamma_{1 i}\left(x_{01}\right)}^{\gamma_{1 i}\left(x_{1}\right)} \int_{\gamma_{2 i}\left(x_{02}\right)}^{\gamma_{2 i}\left(x_{2}\right)} f_{i}\left(x_{1}, t_{1}, x_{2}, t_{1}\right) \\
& \times w_{3}\left(w^{-1}\left(G_{1}^{-1}\left(\zeta_{2}\left(t_{1}, t_{2}\right)\right)\right)\right) \\
& \times\left\{1+\int_{\gamma_{1 i}\left(x_{01}\right)}^{t_{1}} \int_{\gamma_{2 i}\left(x_{02}\right)}^{t_{2}} g_{i}\left(t_{1}, m_{1}, t_{2}, m_{2}\right) \Delta m_{2} \Delta m_{1}\right\} \Delta t_{2} \Delta t_{1} .
\end{aligned}
$$

Let

$$
\begin{aligned}
\vartheta\left(x_{1}, x_{2}\right)= & d_{1}\left(\bar{x}_{1}, \bar{x}_{2}\right)+a_{2}\left(\bar{x}_{1}, \bar{x}_{2}\right) \sum_{i=1}^{n} \int_{\gamma_{1 i}\left(x_{01}\right)}^{\gamma_{1 i}\left(x_{1}\right)} \int_{\gamma_{2 i}\left(x_{02}\right)}^{\gamma_{2 i}\left(x_{2}\right)} f_{i}\left(x_{1}, t_{1}, x_{2}, t_{1}\right) \\
& \times w_{3}\left(w^{-1}\left(G_{1}^{-1}\left(\zeta_{2}\left(t_{1}, t_{2}\right)\right)\right)\right) \\
& \times\left\{1+\int_{\gamma_{1 i}\left(x_{01}\right)}^{t_{1}} \int_{\gamma_{2 i}\left(x_{02}\right)}^{t_{2}} g_{i}\left(t_{1}, m_{1}, t_{2}, m_{2}\right) \Delta m_{2} \Delta m_{1}\right\} \Delta t_{2} \Delta t_{1} .
\end{aligned}
$$

Then

$$
\vartheta\left(x_{01}, x_{2}\right)=\vartheta\left(x_{1}, x_{02}\right)=d_{1}\left(\bar{x}_{1}, \bar{x}_{2}\right)
$$

and

$$
\zeta_{2}\left(x_{1}, x_{2}\right) \leq G_{2}^{-1}\left(\vartheta\left(x_{1}, x_{2}\right)\right)
$$

It follows from Lemma 2.1 and (3.23) that

$$
\begin{aligned}
\vartheta^{\Delta x_{1}}\left(x_{1}, x_{2}\right) \\
=a_{2}\left(\bar{x}_{1}, \bar{x}_{2}\right) \sum_{i=1}^{n}\left[\gamma_{1 i}^{\Delta}\left(x_{1}\right) \int_{\gamma_{2 i}\left(x_{02}\right)}^{\gamma_{2 i}\left(x_{2}\right)} f_{i}\left(\sigma\left(x_{1}\right), \gamma_{1 i}\left(x_{1}\right), x_{2}, t_{2}\right)\right. \\
\quad \times w_{3}\left(w^{-1}\left(G_{1}^{-1}\left(\zeta_{2}\left(\gamma_{1 i}\left(x_{1}\right), t_{2}\right)\right)\right)\right) \\
\quad \times\left\{1+\int_{\gamma_{1 i}\left(x_{01}\right)}^{\gamma_{1 i}\left(x_{1}\right)} \int_{\gamma_{2 i}\left(x_{02}\right)}^{t_{2}} g_{i}\left(\gamma_{1 i}\left(x_{1}\right), m_{1}, t_{2}, m_{2}\right) \Delta m_{2} \Delta m_{1}\right\} \Delta t_{2} \\
+\int_{\gamma_{1 i}\left(x_{01}\right)}^{\gamma_{1 i}\left(x_{1}\right)}\left[\int_{\gamma_{2 i}\left(x_{02}\right)}^{\gamma_{2 i}\left(x_{2}\right)} f_{i}\left(x_{1}, t_{1}, x_{2}, t_{1}\right) w_{3}\left(w^{-1}\left(G_{1}^{-1}\left(\zeta_{2}\left(t_{1}, t_{2}\right)\right)\right)\right)\right. \\
\left.\left.\quad \times\left\{1+\int_{\gamma_{1 i}\left(x_{01}\right)}^{t_{1}} \int_{\gamma_{2 i}\left(x_{02}\right)}^{t_{2}} g_{i}\left(t_{1}, m_{1}, t_{2}, m_{2}\right) \Delta m_{2} \Delta m_{1}\right\} \Delta t_{2}\right]^{\Delta x_{1}} \Delta t_{1}\right] .
\end{aligned}
$$

From (3.25) and the fact that $w_{3}, w^{-1}, G_{1}^{-1}, G_{2}^{-1}$, and $\vartheta$ are nondecreasing, we have

$$
\begin{aligned}
& \frac{\vartheta^{\Delta x_{1}}\left(x_{1}, x_{2}\right)}{w_{3}\left(w^{-1}\left(G_{1}^{-1}\left(G_{2}^{-1}\left(\vartheta\left(x_{1}, x_{2}\right)\right)\right)\right)\right)} \\
& \leq a_{2}\left(\bar{x}_{1}, \bar{x}_{2}\right) \sum_{i=1}^{n}\left[\gamma_{1 i}^{\Delta}\left(x_{1}\right) \int_{\gamma_{2 i}\left(x_{02}\right)}^{\gamma_{2 i}\left(x_{2}\right)} f_{i}\left(\sigma\left(x_{1}\right), \gamma_{1 i}\left(x_{1}\right), x_{2}, t_{2}\right)\right.
\end{aligned}
$$




$$
\begin{aligned}
& \times\left\{1+\int_{\gamma_{1 i}\left(x_{01}\right)}^{\gamma_{1 i}\left(x_{1}\right)} \int_{\gamma_{2 i}\left(x_{02}\right)}^{t_{2}} g_{i}\left(\gamma_{1 i}\left(x_{1}\right), m_{1}, t_{2}, m_{2}\right) \Delta m_{2} \Delta m_{1}\right\} \Delta t_{2} \\
& +\int_{\gamma_{1 i}\left(x_{01}\right)}^{\gamma_{1 i}\left(x_{1}\right)}\left[\int_{\gamma_{2 i}\left(x_{02}\right)}^{\gamma_{2 i}\left(x_{2}\right)} f_{i}\left(x_{1}, t_{1}, x_{2}, t_{1}\right)\right. \\
& \left.\left.\times\left\{1+\int_{\gamma_{1 i}\left(x_{01}\right)}^{t_{1}} \int_{\gamma_{2 i}\left(x_{02}\right)}^{t_{2}} g_{i}\left(t_{1}, m_{1}, t_{2}, m_{2}\right) \Delta m_{2} \Delta m_{1}\right\} \Delta t_{2}\right]^{\Delta x_{1}} \Delta t_{1}\right] \\
= & a_{2}\left(\bar{x}_{1}, \bar{x}_{2}\right) \sum_{i=1}^{n}\left[\int_{\gamma_{1 i}\left(x_{01}\right)}^{\gamma_{1 i}\left(x_{1}\right)} \int_{\gamma_{2 i}\left(x_{02}\right)}^{\gamma_{2 i}\left(x_{2}\right)} f_{i}\left(x_{1}, t_{1}, x_{2}, t_{1}\right)\right. \\
& \left.\times\left\{1+\int_{\gamma_{1 i}\left(x_{01}\right)}^{t_{1}} \int_{\gamma_{2 i}\left(x_{02}\right)}^{t_{2}} g_{i}\left(t_{1}, m_{1}, t_{2}, m_{2}\right) \Delta m_{2} \Delta m_{1}\right\} \Delta t_{2} \Delta t_{1}\right]^{\Delta x_{1}} .
\end{aligned}
$$

Using the definition of $Q_{1}$ and (3.24), integrating over $\left[x_{01}, x_{1}\right]$, we get

$$
Q_{1}\left(\vartheta\left(x_{1}, x_{2}\right)\right) \leq Q_{1}\left(d_{1}\left(\bar{x}_{1}, \bar{x}_{2}\right)\right)+a_{2}\left(\bar{x}_{1}, \bar{x}_{2}\right) c\left(x_{1}, x_{2}\right) .
$$

It follows from (3.19), (3.22), (3.25), and (3.26) that

$$
u\left(x_{1}, x_{2}\right) \leq w^{-1}\left(G_{1}^{-1}\left(G_{2}^{-1}\left(Q_{1}^{-1}\left(Q_{1}\left(d_{1}\left(\bar{x}_{1}, \bar{x}_{2}\right)\right)+a_{2}\left(\bar{x}_{1}, \bar{x}_{2}\right) c\left(x_{1}, x_{2}\right)\right)\right)\right)\right) .
$$

Let $x_{1}=\bar{x}_{1}$ and $x_{2}=\bar{x}_{2}$. Then

$$
u\left(\bar{x}_{1}, \bar{x}_{2}\right) \leq w^{-1}\left(G_{1}^{-1}\left(G_{2}^{-1}\left(Q_{1}^{-1}\left(Q_{1}\left(d_{1}\left(\bar{x}_{1}, \bar{x}_{2}\right)\right)+a_{2}\left(\bar{x}_{1}, \bar{x}_{2}\right) c\left(\bar{x}_{1}, \bar{x}_{2}\right)\right)\right)\right)\right) .
$$

Since $x_{01} \leq \bar{x}_{1} \leq \tilde{x}_{1}$ and $x_{02} \leq \bar{x}_{2} \leq \tilde{x}_{2}$ are chosen arbitrarily, so after substituting $\bar{x}_{1}$ and respectively $\bar{x}_{2}$ with $x_{1}$ and $x_{2}$, we obtain the desired result (3.17).

For $w_{2}(\mathfrak{u})<w_{4}(\log (\mathfrak{u}))$, inequality (3.20) can be rewritten as follows:

$$
\begin{aligned}
G_{1}\left(\xi_{2}\left(x_{1}, x_{2}\right)\right) \\
\leq G_{1}\left(a_{1}\left(\bar{x}_{1}, \bar{x}_{2}\right)\right)+a_{2}\left(\bar{x}_{1}, \bar{x}_{2}\right) \sum_{i=1}^{n} \int_{\gamma_{1 i}\left(x_{01}\right)}^{\gamma_{1 i}\left(x_{1}\right)} \int_{\gamma_{2 i}\left(x_{02}\right)}^{\gamma_{2 i}\left(x_{2}\right)} w_{4}\left(\log \left(w^{-1}\left(\xi_{2}\left(t_{1}, t_{2}\right)\right)\right)\right) \\
\quad \times\left[f _ { i } ( x _ { 1 } , t _ { 1 } , x _ { 2 } , t _ { 2 } ) \left\{w_{3}\left(w^{-1}\left(\xi_{2}\left(t_{1}, t_{2}\right)\right)\right)+\int_{\gamma_{1 i}\left(x_{01}\right)}^{t_{1}} \int_{\gamma_{2 i}\left(x_{02}\right)}^{t_{2}} g_{i}\left(t_{1}, m_{1}, t_{2}, m_{2}\right)\right.\right. \\
\left.\left.\quad \times w_{3}\left(w^{-1}\left(\xi_{2}\left(m_{1}, m_{2}\right)\right)\right) \Delta m_{2} \Delta m_{1}\right\}+r_{i}\left(t_{1}, t_{2}\right)\right] \Delta t_{2} \Delta t_{1} \\
\leq \\
G_{1}\left(a_{1}\left(\bar{x}_{1}, \bar{x}_{2}\right)\right)+a_{2}\left(\bar{x}_{1}, \bar{x}_{2}\right) \sum_{i=1}^{n} \int_{\gamma_{1 i}\left(x_{01}\right)}^{\gamma_{1 i}\left(x_{1}\right)} \int_{\gamma_{2 i}\left(x_{02}\right)}^{\gamma_{2 i}\left(x_{2}\right)} w_{4}\left(w^{-1}\left(\xi_{2}\left(t_{1}, t_{2}\right)\right)\right) \\
\quad \times\left[f _ { i } ( x _ { 1 } , t _ { 1 } , x _ { 2 } , t _ { 2 } ) \left\{w_{3}\left(w^{-1}\left(\xi_{2}\left(t_{1}, t_{2}\right)\right)\right)+\int_{\gamma_{1 i}\left(x_{01}\right)}^{t_{1}} \int_{\gamma_{2 i}\left(x_{02}\right)}^{t_{2}} g_{i}\left(t_{1}, m_{1}, t_{2}, m_{2}\right)\right.\right. \\
\left.\left.\quad \times w_{3}\left(w^{-1}\left(\xi_{2}\left(m_{1}, m_{2}\right)\right)\right) \Delta m_{2} \Delta m_{1}\right\}+r_{i}\left(t_{1}, t_{2}\right)\right] \Delta t_{2} \Delta t_{1} .
\end{aligned}
$$

On taking the identical steps as those (3.21)-(3.27), then after substituting $\bar{x}_{1}$ and $\bar{x}_{2}$ with $x_{1}$ and $x_{2}$, we obtain the desired result (3.18). 
Remark 3.9 Let $\mathbb{T}=\mathrm{R}, w_{3}=1, \mu_{j i}=I, f_{i}\left(x_{1}, t_{1}, x_{2}, t_{2}\right)=f_{i}\left(t_{1}, t_{2}\right)$, and $g_{i}=0$. Then Theorem 3.8 leads to Theorem 2 in [115].

Theorem 3.10 Let $a_{j}, r_{i}, w, w_{j}, f_{i}, g_{i}, \gamma_{j i}, \mu_{j i}$, and $\mathfrak{a}$ be defined as in Theorem 3.1, and $\mathrm{L}, \mathrm{M}: \mathbb{T}_{1} \times \mathbb{T}_{2} \times \mathbb{R}_{0}^{+} \rightarrow \mathbb{R}_{0}^{+}$be right-dense continuous on $\mathbb{T}_{1} \times \mathbb{T}_{2}$ and continuous on $\mathbb{R}_{0}^{+}$ such that

$$
0 \leq \mathrm{L}\left(x_{1}, x_{2}, \mathfrak{u}\right)-\mathrm{L}\left(x_{1}, x_{2}, \mathfrak{v}\right) \leq \mathrm{M}\left(x_{1}, x_{2}, \mathfrak{v}\right)(\mathfrak{u}-\mathfrak{v})
$$

for $\mathfrak{u}>\mathfrak{v} \geq 0$ and $\left(x_{1}, x_{2}\right) \in \mathbb{T}_{1} \times \mathbb{T}_{2}$, and

$$
\begin{aligned}
& w\left(u\left(x_{1}, x_{2}\right)\right) \\
& \leq a_{1}\left(x_{1}, x_{2}\right)+a_{2}\left(x_{1}, x_{2}\right) \sum_{i=1}^{n} \int_{\gamma_{1 i}\left(x_{01}\right)}^{\gamma_{1 i}\left(x_{1}\right)} \int_{\gamma_{2 i}\left(x_{02}\right)}^{\gamma_{2 i}\left(x_{2}\right)} w_{1}\left(u\left(\mu_{1 i}\left(t_{1}\right), \mu_{2 i}\left(t_{2}\right)\right)\right) \\
& \quad \times\left[f _ { i } ( x _ { 1 } , t _ { 1 } , x _ { 2 } , t _ { 2 } ) \left\{w_{2}\left(u\left(\mu_{1 i}\left(t_{1}\right), \mu_{2 i}\left(t_{2}\right)\right)\right)+\int_{\gamma_{1 i}\left(x_{01}\right)}^{t_{1}} \int_{\gamma_{2 i}\left(x_{02}\right)}^{t_{2}} g_{i}\left(t_{1}, m_{1}, t_{2}, m_{2}\right)\right.\right. \\
& \left.\quad \times w_{2}\left(u\left(\mu_{1 i}\left(m_{1}\right), \mu_{2 i}\left(m_{2}\right)\right)\right) \Delta m_{2} \Delta m_{1}\right\} \\
& \left.\quad+r_{i}\left(t_{1}, t_{2}\right) \mathrm{L}\left(t_{1}, t_{2}, w_{2}\left(u\left(t_{1}, t_{2}\right)\right)\right)\right] \Delta t_{2} \Delta t_{1}
\end{aligned}
$$

for $\left(x_{1}, x_{2}\right) \in \mathbb{T}_{1} \times \mathbb{T}_{2}$ with initial condition (3.2). Then

$$
\begin{aligned}
u\left(x_{1}, x_{2}\right) \leq & w^{-1}\left(G _ { 1 } ^ { - 1 } \left(G _ { 2 } ^ { - 1 } \left(G_{2}\left(b_{2}\left(x_{1}, x_{2}\right)\right)+a_{2}\left(x_{1}, x_{2}\right)\left\{c\left(x_{1}, x_{2}\right)\right.\right.\right.\right. \\
& \left.\left.\left.\left.+\sum_{i=1}^{n} \int_{\gamma_{1 i}\left(x_{01}\right)}^{\gamma_{1 i}\left(x_{1}\right)} \int_{\gamma_{2 i}\left(x_{02}\right)}^{\gamma_{2 i}\left(x_{2}\right)} r_{i}\left(t_{1}, t_{2}\right) \mathrm{M}\left(t_{1}, t_{2}, 0\right) \Delta t_{2} \Delta t_{1}\right\}\right)\right)\right)
\end{aligned}
$$

for all $x_{01} \leq x_{1} \leq \tilde{x}_{1}$ and $x_{02} \leq x_{2} \leq \tilde{x}_{2}$, where

$$
\begin{aligned}
b_{2}\left(x_{1}, x_{2}\right)= & G_{1}\left(a_{1}\left(x_{1}, x_{2}\right)\right)+a_{2}\left(x_{1}, x_{2}\right) \\
& \times \sum_{i=1}^{n} \int_{\gamma_{1 i}\left(x_{01}\right)}^{\gamma_{1 i}\left(x_{1}\right)} \int_{\gamma_{2 i}\left(x_{02}\right)}^{\gamma_{2 i}\left(x_{2}\right)} r_{i}\left(t_{1}, t_{2}\right) \mathrm{L}\left(t_{1}, t_{2}, 0\right) \Delta t_{2} \Delta t_{1},
\end{aligned}
$$

$c, G_{1}$, and $G_{2}$ are as defined in Theorem 3.1, and $\tilde{x}_{1}$ and $\tilde{x}_{2}$ are chosen such that

$$
\begin{aligned}
& G_{2}\left(b_{2}\left(x_{1}, x_{2}\right)\right)+a_{2}\left(x_{1}, x_{2}\right)\left\{c\left(x_{1}, x_{2}\right)\right. \\
& \left.+\sum_{i=1}^{n} \int_{\gamma_{1 i}\left(x_{01}\right)}^{\gamma_{1 i}\left(x_{1}\right)} \int_{\gamma_{2 i}\left(x_{02}\right)}^{\gamma_{2 i}\left(x_{2}\right)} r_{i}\left(t_{1}, t_{2}\right) \mathrm{M}\left(t_{1}, t_{2}, 0\right) \Delta t_{2} \Delta t_{1}\right\} \in \operatorname{Dom}\left(G_{2}^{-1}\right), \\
& G_{2}^{-1}\left(G_{2}\left(b_{2}\left(x_{1}, x_{2}\right)\right)+a_{2}\left(x_{1}, x_{2}\right)\left\{c\left(x_{1}, x_{2}\right)\right.\right. \\
& \left.\left.+\sum_{i=1}^{n} \int_{\gamma_{1 i}\left(x_{01}\right)}^{\gamma_{1 i}\left(x_{1}\right)} \int_{\gamma_{2 i}\left(x_{02}\right)}^{\gamma_{2 i}\left(x_{2}\right)} r_{i}\left(t_{1}, t_{2}\right) \mathrm{M}\left(t_{1}, t_{2}, 0\right) \Delta t_{2} \Delta t_{1}\right\}\right) \in \operatorname{Dom}\left(G_{1}^{-1}\right),
\end{aligned}
$$




$$
\begin{aligned}
& G_{1}^{-1}\left(G _ { 2 } ^ { - 1 } \left(G_{2}\left(b_{2}\left(x_{1}, x_{2}\right)\right)+a_{2}\left(x_{1}, x_{2}\right)\left\{c\left(x_{1}, x_{2}\right)\right.\right.\right. \\
& \left.\left.\left.\quad+\sum_{i=1}^{n} \int_{\gamma_{1 i}\left(x_{01}\right)}^{\gamma_{1 i}\left(x_{1}\right)} \int_{\gamma_{2 i}\left(x_{02}\right)}^{\gamma_{2 i}\left(x_{2}\right)} r_{i}\left(t_{1}, t_{2}\right) \mathrm{M}\left(t_{1}, t_{2}, 0\right) \Delta t_{2} \Delta t_{1}\right\}\right)\right) \in \operatorname{Dom}\left(w^{-1}\right) .
\end{aligned}
$$

Proof Let $\bar{x}_{1} \in \mathbb{T}_{1}$ and $\bar{x}_{2} \in \mathbb{T}_{2}$ with $x_{01} \leq \bar{x}_{1} \leq \tilde{x}_{1}$ and $x_{02} \leq \bar{x}_{2} \leq \tilde{x}_{2}$. Then inequality (3.28) can be rewritten as follows:

$$
\begin{aligned}
& w\left(u\left(x_{1}, x_{2}\right)\right) \\
& \leq a_{1}\left(\bar{x}_{1}, \bar{x}_{2}\right)+a_{2}\left(\bar{x}_{1}, \bar{x}_{2}\right) \sum_{i=1}^{n} \int_{\gamma_{1 i}\left(x_{01}\right)}^{\gamma_{1 i}\left(x_{1}\right)} \int_{\gamma_{2 i}\left(x_{02}\right)}^{\gamma_{2 i}\left(x_{2}\right)} w_{1}\left(u\left(\mu_{1 i}\left(t_{1}\right), \mu_{2 i}\left(t_{2}\right)\right)\right) \\
& \quad \times\left[f _ { i } ( x _ { 1 } , t _ { 1 } , x _ { 2 } , t _ { 2 } ) \left\{w_{2}\left(u\left(\mu_{1 i}\left(t_{1}\right), \mu_{2 i}\left(t_{2}\right)\right)\right)+\int_{\gamma_{1 i}\left(x_{01}\right)}^{t_{1}} \int_{\gamma_{2 i}\left(x_{02}\right)}^{t_{2}} g_{i}\left(t_{1}, m_{1}, t_{2}, m_{2}\right)\right.\right. \\
& \left.\left.\quad \times w_{2}\left(u\left(\mu_{1 i}\left(m_{1}\right), \mu_{2 i}\left(m_{2}\right)\right)\right) \Delta m_{2} \Delta m_{1}\right\}+r_{i}\left(t_{1}, t_{2}\right) \mathrm{L}\left(t_{1}, t_{2}, w_{2}\left(u\left(t_{1}, t_{2}\right)\right)\right)\right] \Delta t_{2} \Delta t_{1}
\end{aligned}
$$

for $x_{01} \leq x_{1} \leq \bar{x}_{1}$ and $x_{02} \leq x_{2} \leq \bar{x}_{2}$. Let

$$
\begin{aligned}
& \xi_{3}\left(x_{1}, x_{2}\right) \\
& =a_{1}\left(\bar{x}_{1}, \bar{x}_{2}\right)+a_{2}\left(\bar{x}_{1}, \bar{x}_{2}\right) \sum_{i=1}^{n} \int_{\gamma_{1 i}\left(x_{01}\right)}^{\gamma_{1 i}\left(x_{1}\right)} \int_{\gamma_{2 i}\left(x_{02}\right)}^{\gamma_{2 i}\left(x_{2}\right)} w_{1}\left(u\left(\mu_{1 i}\left(t_{1}\right), \mu_{2 i}\left(t_{2}\right)\right)\right) \\
& \quad \times\left[f _ { i } ( x _ { 1 } , t _ { 1 } , x _ { 2 } , t _ { 2 } ) \left\{w_{2}\left(u\left(\mu_{1 i}\left(t_{1}\right), \mu_{2 i}\left(t_{2}\right)\right)\right)+\int_{\gamma_{1 i}\left(x_{01}\right)}^{t_{1}} \int_{\gamma_{2 i}\left(x_{02}\right)}^{t_{2}} g_{i}\left(t_{1}, m_{1}, t_{2}, m_{2}\right)\right.\right. \\
& \left.\left.\quad \times w_{2}\left(u\left(\mu_{1 i}\left(m_{1}\right), \mu_{2 i}\left(m_{2}\right)\right)\right) \Delta m_{2} \Delta m_{1}\right\}+r_{i}\left(t_{1}, t_{2}\right) \mathrm{L}\left(t_{1}, t_{2}, w_{2}\left(u\left(t_{1}, t_{2}\right)\right)\right)\right] \Delta t_{2} \Delta t_{1} .
\end{aligned}
$$

Then

$$
\xi_{3}\left(x_{01}, x_{2}\right)=\xi_{3}\left(x_{1}, x_{02}\right)=a_{1}\left(\bar{x}_{1}, \bar{x}_{2}\right)
$$

and

$$
u\left(x_{1}, x_{2}\right) \leq w^{-1}\left(\xi_{3}\left(x_{1}, x_{2}\right)\right)
$$

On taking the identical steps as in (3.17)-(3.10), one has

$$
\begin{aligned}
& G_{1}\left(\xi_{3}\left(x_{1}, x_{2}\right)\right) \\
& \leq G_{1}\left(a_{1}\left(\bar{x}_{1}, \bar{x}_{2}\right)\right)+a_{2}\left(\bar{x}_{1}, \bar{x}_{2}\right) \sum_{i=1}^{n} \int_{\gamma_{1 i}\left(x_{01}\right)}^{\gamma_{1 i}\left(x_{1}\right)} \int_{\gamma_{2 i}\left(x_{02}\right)}^{\gamma_{2 i}\left(x_{2}\right)}\left[f_{i}\left(x_{1}, t_{1}, x_{2}, t_{2}\right)\right. \\
& \quad \times\left\{w_{2}\left(w^{-1}\left(\xi_{3}\left(t_{1}, t_{2}\right)\right)\right)+\int_{\gamma_{1 i}\left(x_{01}\right)}^{t_{1}} \int_{\gamma_{2 i}\left(x_{02}\right)}^{t_{2}} g_{i}\left(t_{1}, m_{1}, t_{2}, m_{2}\right)\right. \\
& \left.\quad \times w_{2}\left(w^{-1}\left(\xi_{3}\left(m_{1}, m_{2}\right)\right)\right) \Delta m_{2} \Delta m_{1}\right\}
\end{aligned}
$$




$$
\begin{aligned}
& \left.+r_{i}\left(t_{1}, t_{2}\right) \mathrm{L}\left(t_{1}, t_{2}, w_{2}\left(w^{-1}\left(\xi_{3}\left(t_{1}, t_{2}\right)\right)\right)\right)\right] \Delta t_{2} \Delta t_{1} \\
\leq & G_{1}\left(a_{1}\left(\bar{x}_{1}, \bar{x}_{2}\right)\right)+a_{2}\left(\bar{x}_{1}, \bar{x}_{2}\right) \sum_{i=1}^{n} \int_{\gamma_{1 i}\left(x_{01}\right)}^{\gamma_{1 i}\left(x_{1}\right)} \int_{\gamma_{2 i}\left(x_{02}\right)}^{\gamma_{2 i}\left(x_{2}\right)}\left[f_{i}\left(x_{1}, t_{1}, x_{2}, t_{2}\right)\right. \\
& \times\left\{w_{2}\left(w^{-1}\left(\xi_{3}\left(t_{1}, t_{2}\right)\right)\right)+\int_{\gamma_{1 i}\left(x_{01}\right)}^{t_{1}} \int_{\gamma_{2 i}\left(x_{02}\right)}^{t_{2}} g_{i}\left(t_{1}, m_{1}, t_{2}, m_{2}\right)\right. \\
& \left.\times w_{2}\left(w^{-1}\left(\xi_{3}\left(m_{1}, m_{2}\right)\right)\right) \Delta m_{2} \Delta m_{1}\right\} \\
& +r_{i}\left(t_{1}, t_{2}\right)\left\{\mathrm{L}\left(t_{1}, t_{2}, 0\right)+\mathrm{M}\left(t_{1}, t_{2}, 0\right) w_{2}\left(w^{-1}\left(\xi_{3}\left(t_{1}, t_{2}\right)\right)\right)\right\} \Delta t_{2} \Delta t_{1} \\
\leq & G_{1}\left(a_{1}\left(\bar{x}_{1}, \bar{x}_{2}\right)\right)+a_{2}\left(\bar{x}_{1}, \bar{x}_{2}\right) \sum_{i=1}^{n} \int_{\gamma_{1 i}\left(x_{01}\right)}^{\gamma_{1 i}\left(\bar{x}_{1}\right)} \int_{\gamma_{2 i}\left(x_{02}\right)}^{\gamma_{2 i}\left(\bar{x}_{2}\right)} r_{i}\left(t_{1}, t_{2}\right) \mathrm{L}\left(t_{1}, t_{2}, 0\right) \Delta t_{2} \Delta t_{1} \\
+ & b_{2}\left(\bar{x}_{1}, \bar{x}_{2}\right)+a_{2}\left(\bar{x}_{1}, \bar{x}_{2}\right) \sum_{i=1}^{n} \int_{\gamma_{1 i}\left(x_{01}\right)}^{\gamma_{1 i}\left(x_{1}\right)} \int_{\gamma_{2 i}\left(x_{02}\right)}^{\gamma_{2 i}\left(x_{2}\right)} w_{2}\left(w^{-1}\left(\xi_{3}\left(t_{1}, t_{2}\right)\right)\right) \\
& +\left[f_{i}\left(x_{1}, t_{1}, x_{2}, t_{2}\right)\left\{1+\int_{\gamma_{1 i}\left(x_{01}\right)}^{t_{1}} \int_{\gamma_{2 i}\left(x_{02}\right)}^{t_{2}} g_{i}\left(t_{1}, m_{1}, t_{2}, m_{2}\right) \Delta m_{2} \Delta m_{1}\right\}\right. \\
& \left.\left.+a_{2}\left(\bar{x}_{1}, \bar{x}_{2}\right) \sum_{i=1}^{n} \int_{\gamma_{1 i}\left(x_{01}\right)}^{\gamma_{1 i}\left(x_{1}\right)} \int_{\gamma_{2 i}\left(x_{02}\right)}^{\gamma_{2 i}\left(x_{2}\right)} w_{2}\left(t_{1}, t_{2}\right) \mathrm{M}\left(t_{1}, t_{2}, 0\right)\right] \Delta t_{2}\left(\xi_{3}\left(t_{1}, t_{2}\right)\right)\right) \\
& \times\left[f_{i}\left(x_{1}, t_{1}, x_{2}, t_{2}\right)\left\{1+\int_{\gamma_{1 i}\left(x_{01}\right)}^{t_{1}} \int_{\gamma_{2 i}\left(x_{02}\right)}^{t_{2}} g_{i}\left(t_{1}, m_{1}, t_{2}, m_{2}\right) \Delta m_{2} \Delta m_{1}\right\}\right.
\end{aligned}
$$

Let

$$
\begin{aligned}
\zeta_{3}\left(x_{1}, x_{2}\right)= & b_{2}\left(\bar{x}_{1}, \bar{x}_{2}\right)+a_{2}\left(\bar{x}_{1}, \bar{x}_{2}\right) \sum_{i=1}^{n} \int_{\gamma_{1 i}\left(x_{01}\right)}^{\gamma_{1 i}\left(x_{1}\right)} \int_{\gamma_{2 i}\left(x_{02}\right)}^{\gamma_{2 i}\left(x_{2}\right)} w_{2}\left(w^{-1}\left(\xi_{3}\left(t_{1}, t_{2}\right)\right)\right) \\
& \times\left[f_{i}\left(x_{1}, t_{1}, x_{2}, t_{2}\right)\left\{1+\int_{\gamma_{1 i}\left(x_{01}\right)}^{t_{1}} \int_{\gamma_{2 i}\left(x_{02}\right)}^{t_{2}} g_{i}\left(t_{1}, m_{1}, t_{2}, m_{2}\right) \Delta m_{2} \Delta m_{1}\right\}\right. \\
& \left.+r_{i}\left(t_{1}, t_{2}\right) \mathrm{M}\left(t_{1}, t_{2}, 0\right)\right] \Delta t_{2} \Delta t_{1} .
\end{aligned}
$$

Then

$$
\zeta_{3}\left(x_{01}, x_{2}\right)=\zeta_{3}\left(x_{1}, x_{02}\right)=b_{2}\left(\bar{x}_{1}, \bar{x}_{2}\right)
$$

and

$$
\xi_{3}\left(x_{1}, x_{2}\right) \leq G_{1}^{-1}\left(\zeta_{3}\left(x_{1}, x_{2}\right)\right)
$$


On taking the identical steps as in (3.13) to (3.14), one has

$$
\begin{aligned}
& G_{2}\left(\zeta_{3}\left(x_{1}, x_{2}\right)\right) \\
& \leq G_{2}\left(b_{2}\left(\bar{x}_{1}, \bar{x}_{2}\right)\right)+a_{2}\left(\bar{x}_{1}, \bar{x}_{2}\right) \sum_{i=1}^{n} \int_{\gamma_{1 i}\left(x_{01}\right)}^{\gamma_{1 i}\left(x_{1}\right)} \int_{\gamma_{2 i}\left(x_{02}\right)}^{\gamma_{2 i}\left(x_{2}\right)}\left\{f_{i}\left(x_{1}, t_{1}, x_{2}, t_{2}\right)\right. \\
& \quad \times\left(1+\int_{\gamma_{1 i}\left(x_{01}\right)}^{t_{1}} \int_{\gamma_{2 i}\left(x_{02}\right)}^{t_{2}} g_{i}\left(t_{1}, m_{1}, t_{2}, m_{2}\right) \Delta m_{2} \Delta m_{1}\right) \\
& \left.\quad+r_{i}\left(t_{1}, t_{2}\right) \mathrm{M}\left(t_{1}, t_{2}, 0\right)\right\} \Delta t_{2} \Delta t_{1} \\
& =G_{2}\left(b_{2}\left(\bar{x}_{1}, \bar{x}_{2}\right)\right)+a_{2}\left(\bar{x}_{1}, \bar{x}_{2}\right)\left\{c\left(x_{1}, x_{2}\right)\right. \\
& \left.\quad+\sum_{i=1}^{n} \int_{\gamma_{1 i}\left(x_{01}\right)}^{\gamma_{1 i}\left(x_{1}\right)} \int_{\gamma_{2 i}\left(x_{02}\right)}^{\gamma_{2 i}\left(x_{2}\right)} r_{i}\left(t_{1}, t_{2}\right) \mathrm{M}\left(t_{1}, t_{2}, 0\right) \Delta t_{2} \Delta t_{1}\right\} .
\end{aligned}
$$

It follows from (3.30)-(3.32) that

$$
\begin{aligned}
u\left(x_{1}, x_{2}\right) \leq & w^{-1}\left(G _ { 1 } ^ { - 1 } \left(G _ { 2 } ^ { - 1 } \left(G_{2}\left(b_{2}\left(\bar{x}_{1}, \bar{x}_{2}\right)\right)+a_{2}\left(\bar{x}_{1}, \bar{x}_{2}\right)\left\{c\left(x_{1}, x_{2}\right)\right.\right.\right.\right. \\
& \left.\left.\left.\left.+\sum_{i=1}^{n} \int_{\gamma_{1 i}\left(x_{01}\right)}^{\gamma_{1 i}\left(x_{1}\right)} \int_{\gamma_{2 i}\left(x_{02}\right)}^{\gamma_{2 i}\left(x_{2}\right)} r_{i}\left(t_{1}, t_{2}\right) \mathrm{M}\left(t_{1}, t_{2}, 0\right) \Delta t_{2} \Delta t_{1}\right\}\right)\right)\right) .
\end{aligned}
$$

Let $x_{1}=\bar{x}_{1}$ and $x_{2}=\bar{x}_{2}$. Then we have

$$
\begin{aligned}
u\left(\bar{x}_{1}, \bar{x}_{2}\right) \leq & w^{-1}\left(G _ { 1 } ^ { - 1 } \left(G _ { 2 } ^ { - 1 } \left(G_{2}\left(b_{2}\left(\bar{x}_{1}, \bar{x}_{2}\right)\right)+a_{2}\left(\bar{x}_{1}, \bar{x}_{2}\right)\left\{c\left(\bar{x}_{1}, \bar{x}_{2}\right)\right.\right.\right.\right. \\
& \left.\left.\left.\left.+\sum_{i=1}^{n} \int_{\gamma_{1 i}\left(x_{01}\right)}^{\gamma_{1 i}\left(\bar{x}_{1}\right)} \int_{\gamma_{2 i}\left(x_{02}\right)}^{\gamma_{2 i}\left(\bar{x}_{2}\right)} r_{i}\left(t_{1}, t_{2}\right) \mathrm{M}\left(t_{1}, t_{2}, 0\right) \Delta t_{2} \Delta t_{1}\right\}\right)\right)\right) .
\end{aligned}
$$

Since $x_{01} \leq \bar{x}_{1} \leq \tilde{x}_{1}$ and $x_{02} \leq \bar{x}_{2} \leq \tilde{x}_{2}$ are chosen arbitrarily, we obtain

$$
\begin{aligned}
u\left(x_{1}, x_{2}\right) \leq & w^{-1}\left(G _ { 1 } ^ { - 1 } \left(G _ { 2 } ^ { - 1 } \left(G_{2}\left(b_{2}\left(x_{1}, x_{2}\right)\right)+a_{2}\left(x_{1}, x_{2}\right)\left\{c\left(x_{1}, x_{2}\right)\right.\right.\right.\right. \\
& \left.\left.\left.\left.+\sum_{i=1}^{n} \int_{\gamma_{1 i}\left(x_{01}\right)}^{\gamma_{1 i}\left(x_{1}\right)} \int_{\gamma_{2 i}\left(x_{02}\right)}^{\gamma_{2 i}\left(x_{2}\right)} r_{i}\left(t_{1}, t_{2}\right) \mathrm{M}\left(t_{1}, t_{2}, 0\right) \Delta t_{2} \Delta t_{1}\right\}\right)\right)\right) .
\end{aligned}
$$

Remark 3.11 Let $\mathbb{T}=\mathbb{R}, w_{2}=\mu_{j i}=I, f_{i}\left(x_{1}, t_{1}, x_{2}, t_{2}\right)=f_{i}\left(t_{1}, t_{2}\right)$, and $g_{i}=0$. Then Theorem 3.10 coincides with Theorem 3 in [115].

Corollary 3.12 Let $u, r_{i}, w_{2}, f_{i}, g_{i}, \gamma_{j i}$, and $\mu_{j i}$ be defined as in Theorem 3.1, $\overline{\mathfrak{a}}\left(x_{1}, x_{2}\right)$ be a nonnegative and right-dense continuous function defined on $\left(\left[\overline{\mathfrak{p}}_{1}, x_{01}\right] \times\left[\overline{\mathfrak{p}}_{2}, x_{02}\right]\right)_{\mathbb{T}^{2}}$, and 
$q_{1}>q_{2}>0$ and $\mathfrak{C} \geq 0$ be constants such that

$$
\begin{aligned}
u^{q_{1}}\left(x_{1}, x_{2}\right) \leq & \mathfrak{C}+\frac{q_{1}}{q_{1}-q_{2}} \sum_{i=1}^{n} \int_{\gamma_{1 i}\left(x_{01}\right)}^{\gamma_{1 i}\left(x_{1}\right)} \int_{\gamma_{2 i}\left(x_{02}\right)}^{\gamma_{2 i}\left(x_{2}\right)} u^{q_{2}}\left(\mu_{1 i}\left(t_{1}\right), \mu_{2 i}\left(t_{2}\right)\right) \\
& \times\left[f _ { i } ( x _ { 1 } , t _ { 1 } , x _ { 2 } , t _ { 2 } ) \left\{w_{2}\left(u\left(\mu_{1 i}\left(t_{1}\right), \mu_{2 i}\left(t_{2}\right)\right)\right)\right.\right. \\
& +\int_{\gamma_{1 i}\left(x_{01}\right)}^{t_{1}} \int_{\gamma_{2 i}\left(x_{02}\right)}^{t_{2}} g_{i}\left(t_{1}, m_{1}, t_{2}, m_{2}\right) \\
& \left.\left.\times w_{2}\left(u\left(\mu_{1 i}\left(m_{1}\right), \mu_{2 i}\left(m_{2}\right)\right)\right) \Delta m_{2} \Delta m_{1}\right\}+r_{i}\left(t_{1}, t_{2}\right)\right] \Delta t_{2} \Delta t_{1}
\end{aligned}
$$

for $\left(x_{1}, x_{2}\right) \in \mathbb{T}_{1} \times \mathbb{T}_{2}$ with the initial condition

$$
\left\{\begin{array}{l}
u\left(x_{1}, x_{2}\right)=\overline{\mathfrak{a}}\left(x_{1}, x_{2}\right), \quad x_{1} \in\left[\overline{\mathfrak{p}}_{1}, x_{01}\right]_{\mathbb{T}} \text { or } x_{2} \in\left[\overline{\mathfrak{p}}_{2}, x_{02}\right]_{\mathbb{T}}, \\
\overline{\mathfrak{a}}\left(\mu_{1 i}\left(x_{1}\right), \mu_{2 i}\left(x_{2}\right)\right) \leq \sqrt[q_{1}]{\mathfrak{C},} \quad \mu_{1 i}\left(x_{1}\right) \leq x_{01} \text { or } \mu_{2 i}\left(x_{2}\right) \leq x_{02}
\end{array}\right.
$$

Then

$$
u\left(x_{1}, x_{2}\right) \leq \sqrt[q_{1}-q_{2}]{H_{1}^{-1}\left(H_{1}\left(\bar{b}_{1}\left(x_{1}, x_{2}\right)\right)+c\left(x_{1}, x_{2}\right)\right)}
$$

for all $x_{01} \leq x_{1} \leq \tilde{x}_{1}$ and $x_{02} \leq x_{2} \leq \tilde{x}_{2}$, where

$$
\begin{aligned}
& \bar{b}_{1}\left(x_{1}, x_{2}\right):=\mathfrak{C}^{\frac{q_{1}-q_{2}}{q_{1}}}+\sum_{i=1}^{n} \int_{\gamma_{1 i}\left(x_{01}\right)}^{\gamma_{1 i}\left(x_{1}\right)} \int_{\gamma_{2 i}\left(x_{02}\right)}^{\gamma_{2 i}\left(x_{2}\right)} r_{i}\left(t_{1}, t_{2}\right) \Delta t_{2} \Delta t_{1}, \\
& H_{1}(s)=\int_{s_{6}}^{s} \frac{\Delta p}{w_{2}\left(\sqrt[q_{1}-q_{2}]{p}\right)}, \quad s>s_{6}>0 \text { with } H_{1}(\infty)=\infty,
\end{aligned}
$$

$c$ is defined as in Theorem 3.1, $H_{1}^{-1}$ is the inverse function of $H_{1}$, and $\tilde{x}_{1}$ and $\tilde{x}_{2}$ are chosen such that

$$
H_{1}\left(\bar{b}_{1}\left(x_{1}, x_{2}\right)\right)+c\left(x_{1}, x_{2}\right) \in \operatorname{Dom}\left(H_{1}^{-1}\right) .
$$

Proof Let

$$
\begin{aligned}
\bar{\xi}_{1}\left(x_{1}, x_{2}\right)= & \mathfrak{C}+\frac{q_{1}}{q_{1}-q_{2}} \sum_{i=1}^{n} \int_{\gamma_{1 i}\left(x_{01}\right)}^{\gamma_{1 i}\left(x_{1}\right)} \int_{\gamma_{2 i}\left(x_{02}\right)}^{\gamma_{2 i}\left(x_{2}\right)} u^{q_{2}}\left(\mu_{1 i}\left(t_{1}\right), \mu_{2 i}\left(t_{2}\right)\right) \\
& \times\left[f _ { i } ( x _ { 1 } , t _ { 1 } , x _ { 2 } , t _ { 2 } ) \left\{w_{2}\left(u\left(\mu_{1 i}\left(t_{1}\right), \mu_{2 i}\left(t_{2}\right)\right)\right)\right.\right. \\
& +\int_{\gamma_{1 i}\left(x_{01}\right)}^{t_{1}} \int_{\gamma_{2 i}\left(x_{02}\right)}^{t_{2}} g_{i}\left(t_{1}, m_{1}, t_{2}, m_{2}\right) \\
& \left.\left.\times w_{2}\left(u\left(\mu_{1 i}\left(m_{1}\right), \mu_{2 i}\left(m_{2}\right)\right)\right) \Delta m_{2} \Delta m_{1}\right\}+r_{i}\left(t_{1}, t_{2}\right)\right] \Delta t_{2} \Delta t_{1} .
\end{aligned}
$$

Then

$$
\bar{\xi}_{1}\left(x_{01}, x_{2}\right)=\bar{\xi}_{1}\left(x_{1}, x_{02}\right)=\mathfrak{C}
$$


and

$$
u\left(x_{1}, x_{2}\right) \leq \sqrt[q_{1}]{\bar{\xi}_{1}\left(x_{1}, x_{2}\right)} \text { for } x_{1} \in\left[x_{01}, \bar{x}_{1}\right]_{\mathbb{T}} \text { and } x_{2} \in\left[x_{02}, \bar{x}_{2}\right]_{\mathbb{T}}
$$

where $\bar{x}_{1} \in \mathbb{T}_{1}$ and $\bar{x}_{2} \in \mathbb{T}_{2}$ are fixed numbers with $x_{01} \leq \bar{x}_{1} \leq \tilde{x}_{1}$ and $x_{02} \leq \bar{x}_{2} \leq \tilde{x}_{2}$.

For $x_{1} \in\left[x_{01}, \bar{x}_{1}\right]_{\mathbb{T}}$ and $x_{2} \in\left[x_{02}, \bar{x}_{2}\right]_{\mathbb{T}}$, if $\mu_{1 i}\left(x_{1}\right) \geq x_{01}$ and $\mu_{2 i}\left(x_{2}\right) \geq x_{02}$, then $\mu_{1 i}\left(x_{1}\right) \in$ $\left[x_{01}, \bar{x}_{1}\right]_{\mathbb{T}}, \mu_{2 i}\left(x_{1}\right) \in\left[x_{02}, \bar{x}_{2}\right]_{\mathbb{T}}$, and

$$
u\left(\mu_{1 i}\left(x_{1}\right), \mu_{2 i}\left(x_{2}\right)\right) \leq \sqrt[q_{1}]{\bar{\xi}_{1}\left(\mu_{1 i}\left(x_{1}\right), \mu_{2 i}\left(x_{2}\right)\right)} \leq \sqrt[q_{1}]{\bar{\xi}_{1}\left(x_{1}, x_{2}\right)}
$$

If $\mu_{1 i}\left(x_{1}\right) \leq x_{01}$ or $\mu_{2 i}\left(x_{2}\right) \leq x_{02}$, then from (3.34) we obtain

$$
u\left(\mu_{1 i}\left(x_{1}\right), \mu_{2 i}\left(x_{2}\right)\right)=\overline{\mathfrak{a}}\left(\mu_{1 i}\left(x_{1}\right), \mu_{2 i}\left(x_{2}\right)\right) \leq \sqrt[q_{1}]{\mathfrak{C}} \leq \sqrt[q_{1}]{\bar{\xi}_{1}\left(x_{1}, x_{2}\right)} .
$$

It follows from (3.39) and (3.40) that

$$
u\left(\mu_{1 i}\left(x_{1}\right), \mu_{2 i}\left(x_{2}\right)\right) \leq \sqrt[q_{1}]{\bar{\xi}_{1}\left(x_{1}, x_{2}\right)}
$$

for $x_{1} \in\left[x_{01}, \bar{x}_{1}\right]_{\mathbb{T}}$ and $x_{2} \in\left[x_{02}, \bar{x}_{2}\right]_{\mathbb{T}}$.

From Lemma 2.1 and (3.36) we know that

$$
\begin{aligned}
\bar{\xi}_{1}^{\Delta x_{1}}\left(x_{1}, x_{2}\right) & \frac{q_{1}}{q_{1}-q_{2}} \sum_{i=1}^{n} \gamma_{1 i}^{\Delta}\left(x_{1}\right) \int_{\gamma_{2 i}\left(x_{02}\right)}^{\gamma_{2 i}\left(x_{2}\right)} u^{q_{2}}\left(\mu_{1 i}\left(\gamma_{1 i}\left(x_{1}\right)\right), \mu_{2 i}\left(t_{2}\right)\right) \\
& \times\left[f _ { i } ( \sigma ( x _ { 1 } ) , \gamma _ { 1 i } ( x _ { 1 } ) , x _ { 2 } , t _ { 2 } ) \left\{w_{2}\left(u\left(\mu_{1 i}\left(\gamma_{1 i}\left(x_{1}\right)\right), \mu_{2 i}\left(t_{2}\right)\right)\right)\right.\right. \\
& +\int_{\gamma_{1 i}\left(x_{01}\right)}^{\gamma_{1 i}\left(x_{1}\right)} \int_{\gamma_{2 i}\left(x_{02}\right)}^{t_{2}} g_{i}\left(\gamma_{1 i}\left(x_{1}\right), m_{1}, t_{2}, m_{2}\right) \\
& \left.\left.\times w_{2}\left(u\left(\mu_{1 i}\left(m_{1}\right), \mu_{2 i}\left(m_{2}\right)\right)\right) \Delta m_{2} \Delta m_{1}\right\}+r_{i}\left(\gamma_{1 i}\left(x_{1}\right), t_{2}\right)\right] \Delta t_{2} \\
& +\frac{q_{1}}{q_{1}-q_{2}} \sum_{i=1}^{n} \int_{\gamma_{1 i}\left(x_{01}\right)}^{\gamma_{1 i}\left(x_{1}\right)}\left[\int_{\gamma_{2 i}\left(x_{02}\right)}^{\gamma_{2 i}\left(x_{2}\right)} u^{q_{2}}\left(\mu_{1 i}\left(t_{1}\right), \mu_{2 i}\left(t_{2}\right)\right)\right. \\
& \times\left[f _ { i } ( x _ { 1 } , t _ { 1 } , x _ { 2 } , t _ { 2 } ) \left\{w_{2}\left(u\left(\mu_{1 i}\left(t_{1}\right), \mu_{2 i}\left(t_{2}\right)\right)\right)+\int_{\gamma_{1 i}\left(x_{01}\right)}^{t_{1}} \int_{\gamma_{2 i}\left(x_{02}\right)}^{t_{2}} g_{i}\left(t_{1}, m_{1}, t_{2}, m_{2}\right)\right.\right. \\
& \left.\left.\left.\times w_{2}\left(u\left(\mu_{1 i}\left(m_{1}\right), \mu_{2 i}\left(m_{2}\right)\right)\right) \Delta m_{2} \Delta m_{1}\right\}+r_{i}\left(t_{1}, t_{2}\right)\right] \Delta t_{2}\right]^{\Delta x_{1}} \Delta t_{1} .
\end{aligned}
$$

Making use of (3.41) and the fact that $\bar{\xi}_{1}$ is nondecreasing, we have

$$
\begin{aligned}
& \frac{\bar{\xi}_{1}^{\Delta x_{1}}\left(x_{1}, x_{2}\right)}{\bar{\xi}_{1}^{\frac{q_{2}}{q_{1}}}\left(x_{1}, x_{2}\right)} \\
& \quad \leq \frac{q_{1}}{q_{1}-q_{2}} \sum_{i=1}^{n} \gamma_{1 i}^{\Delta}\left(x_{1}\right) \int_{\gamma_{2 i}\left(x_{02}\right)}^{\gamma_{2 i}\left(x_{2}\right)}\left[f_{i}\left(\sigma\left(x_{1}\right), \gamma_{1 i}\left(x_{1}\right), x_{2}, t_{2}\right)\right.
\end{aligned}
$$




$$
\begin{aligned}
& \times\left\{w_{2}\left(\sqrt[q_{1}]{\bar{\xi}_{1}\left(\gamma_{1 i}\left(x_{1}\right), t_{2}\right)}\right)+\int_{\gamma_{1 i}\left(x_{01}\right)}^{\gamma_{1 i}\left(x_{1}\right)} \int_{\gamma_{2 i}\left(x_{02}\right)}^{t_{2}} g_{i}\left(\gamma_{1 i}\left(x_{1}\right), m_{1}, t_{2}, m_{2}\right)\right. \\
& \left.\left.\times w_{2}\left(\sqrt[q_{1}]{\bar{\xi}_{1}\left(m_{1}, m_{2}\right)}\right) \Delta m_{2} \Delta m_{1}\right\}+r_{i}\left(\gamma_{1 i}\left(x_{1}\right), t_{2}\right)\right] \Delta t_{2} \\
& +\frac{q_{1}}{q_{1}-q_{2}} \sum_{i=1}^{n} \int_{\gamma_{1 i}\left(x_{01}\right)}^{\gamma_{1 i}\left(x_{1}\right)}\left[\int _ { \gamma _ { 2 i } ( x _ { 0 2 } ) } ^ { \gamma _ { 2 i } ( x _ { 2 } ) } \left[f _ { i } ( x _ { 1 } , t _ { 1 } , x _ { 2 } , t _ { 2 } ) \left\{w_{2}\left(\sqrt[q_{1}]{\bar{\xi}_{1}\left(t_{1}, t_{2}\right)}\right)\right.\right.\right. \\
& \left.+\int_{\gamma_{1 i}\left(x_{01}\right)}^{t_{1}} \int_{\gamma_{2 i}\left(x_{02}\right)}^{t_{2}} g_{i}\left(t_{1}, m_{1}, t_{2}, m_{2}\right) w_{2}\left(\sqrt[q_{1}]{\bar{\xi}_{1}\left(m_{1}, m_{2}\right)}\right) \Delta m_{2} \Delta m_{1}\right\} \\
& \left.\left.+r_{i}\left(t_{1}, t_{2}\right)\right] \Delta t_{2}\right]^{\Delta x_{1}} \Delta t_{1} \\
& =\frac{q_{1}}{q_{1}}-q_{2} \sum_{i=1}^{n}\left[\int _ { \gamma _ { 1 i } ( x _ { 0 1 } ) } ^ { \gamma _ { 1 i } ( x _ { 1 } ) } \int _ { \gamma _ { 2 i } ( x _ { 0 2 } ) } ^ { \gamma _ { 2 i } ( x _ { 2 } ) } \left[f _ { i } ( x _ { 1 } , t _ { 1 } , x _ { 2 } , t _ { 2 } ) \left\{w_{2}\left(\sqrt[q_{1}]{\xi_{1}\left(t_{1}, t_{2}\right)}\right)\right.\right.\right. \\
& \left.+\int_{\gamma_{1 i}\left(x_{01}\right)}^{t_{1}} \int_{\gamma_{2 i}\left(x_{02}\right)}^{t_{2}} g_{i}\left(t_{1}, m_{1}, t_{2}, m_{2}\right) w_{2}\left(\sqrt[q_{1}]{\bar{\xi}_{1}\left(m_{1}, m_{2}\right)}\right) \Delta m_{2} \Delta m_{1}\right\} \\
& \left.\left.+r_{i}\left(t_{1}, t_{2}\right)\right] \Delta t_{2} \Delta t_{1}\right]^{\Delta x_{1}} .
\end{aligned}
$$

Theorem 2.2 and $\bar{\xi}_{1}^{\Delta_{x_{1}}}\left(x_{1}, x_{2}\right) \geq 0$ lead to the conclusion that

$$
\begin{aligned}
& \left(\frac{q_{1}}{q_{1}-q_{2}} \bar{\xi}_{1}^{\frac{q_{1}-q_{2}}{q_{1}}}\left(x_{1}, x_{2}\right)\right)^{\Delta x_{1}} \\
& =\bar{\xi}_{1}^{\Delta x_{1}}\left(x_{1}, x_{2}\right) \int_{0}^{1}\left\{\bar{\xi}_{1}\left(x_{1}, x_{2}\right)+h \mu\left(x_{1}, x_{2}\right) \bar{\xi}_{1}^{\Delta x_{1}}\left(x_{1}, x_{2}\right)\right\}^{-\frac{q_{2}}{q_{1}}} d h \\
& =\frac{\bar{\xi}_{1}^{\Delta x_{1}}\left(x_{1}, x_{2}\right)}{\bar{\xi}_{1}^{\frac{q_{2}}{q_{1}}}\left(x_{1}, x_{2}\right)} \int_{0}^{1}\left\{1+h \mu\left(x_{1}, x_{2}\right) \frac{\bar{\xi}_{1}^{\Delta x_{1}}\left(x_{1}, x_{2}\right)}{\bar{\xi}_{1}\left(x_{1}, x_{2}\right)}\right\}^{-\frac{q_{2}}{q_{1}}} d h \\
& =\frac{\bar{\xi}_{1}^{\Delta x_{1}}\left(x_{1}, x_{2}\right)}{\bar{\xi}_{1}^{\frac{q_{2}}{q_{1}}}\left(x_{1}, x_{2}\right)} \frac{\left\{1+\mu\left(x_{1}, x_{2}\right) \frac{\bar{\xi}_{1}^{\Delta x_{1}}\left(x_{1}, x_{2}\right)}{\bar{\xi}_{1}\left(x_{1}, x_{2}\right)}\right\}^{-\frac{q_{2}}{q_{1}}+1}-1}{\mu\left(x_{1}, x_{2}\right) \frac{\bar{\xi}_{1}^{\Delta x_{1}}\left(x_{1}, x_{2}\right)}{\bar{\xi}_{1}\left(x_{1}, x_{2}\right)}\left(1-\frac{q_{2}}{q_{1}}\right)} \leq \frac{\bar{\xi}_{1}^{\Delta x_{1}}\left(x_{1}, x_{2}\right)}{\bar{\xi}_{1}^{\frac{q_{2}}{q_{1}}}\left(x_{1}, x_{2}\right)}
\end{aligned}
$$

From (3.42) and (3.43) we have

$$
\begin{aligned}
& \left(\frac{q_{1}}{q_{1}-q_{2}} \bar{\xi}_{1}^{\frac{q_{1}-q_{2}}{q_{1}}}\left(x_{1}, x_{2}\right)\right)^{\Delta x_{1}} \\
& \leq \frac{q_{1}}{q_{1}-q_{2}} \sum_{i=1}^{n}\left[\int _ { \gamma _ { 1 i } ( x _ { 0 1 } ) } ^ { \gamma _ { 1 i } ( x _ { 1 } ) } \int _ { \gamma _ { 2 i } ( x _ { 0 2 } ) } ^ { \gamma _ { 2 i } ( x _ { 2 } ) } \left[f_{i}\left(x_{1}, t_{1}, x_{2}, t_{2}\right)\right.\right. \\
& \quad \times\left\{w_{2}\left(\sqrt[q_{1}]{\bar{\xi}_{1}\left(t_{1}, t_{2}\right)}\right)+\int_{\gamma_{1 i}\left(x_{01}\right)}^{t_{1}} \int_{\gamma_{2 i}\left(x_{02}\right)}^{t_{2}} g_{i}\left(t_{1}, m_{1}, t_{2}, m_{2}\right)\right. \\
& \left.\left.\left.\quad \times w_{2}\left(\sqrt[q_{1}]{\bar{\xi}_{1}\left(m_{1}, m_{2}\right)}\right) \Delta m_{2} \Delta m_{1}\right\}+r_{i}\left(t_{1}, t_{2}\right)\right] \Delta t_{2} \Delta t_{1}\right]^{\Delta x_{1}} .
\end{aligned}
$$


Making use of (3.37) and integrating over $\left[x_{01}, x_{1}\right]$, we get

$$
\begin{aligned}
& \bar{\xi}_{1}^{\frac{q_{1}-q_{2}}{q_{1}}}\left(x_{1}, x_{2}\right) \leq \mathfrak{C}^{\frac{q_{1}-q_{2}}{q_{1}}}+\sum_{i=1}^{n} \int_{\gamma_{1 i}\left(x_{01}\right)}^{\gamma_{1 i}\left(x_{1}\right)} \int_{\gamma_{2 i}\left(x_{02}\right)}^{\gamma_{2 i}\left(x_{2}\right)} r_{i}\left(t_{1}, t_{2}\right) \Delta t_{2} \Delta t_{1} \\
& +\sum_{i=1}^{n} \int_{\gamma_{1 i}\left(x_{01}\right)}^{\gamma_{1 i}\left(x_{1}\right)} \int_{\gamma_{2 i}\left(x_{02}\right)}^{\gamma_{2 i}\left(x_{2}\right)} f_{i}\left(x_{1}, t_{1}, x_{2}, t_{2}\right) \\
& \times\left\{w_{2}\left(\sqrt[q_{1}]{\bar{\xi}_{1}\left(t_{1}, t_{2}\right)}\right)+\int_{\gamma_{1 i}\left(x_{01}\right)}^{t_{1}} \int_{\gamma_{2 i}\left(x_{02}\right)}^{t_{2}} g_{i}\left(t_{1}, m_{1}, t_{2}, m_{2}\right)\right. \\
& \left.\times w_{2}\left(\sqrt[q_{1}]{\bar{\xi}_{1}\left(m_{1}, m_{2}\right)}\right) \Delta m_{2} \Delta m_{1}\right\} \Delta t_{2} \Delta t_{1} \\
& \leq \mathfrak{C}^{\frac{q_{1}-q_{2}}{q_{1}}}+\sum_{i=1}^{n} \int_{\gamma_{1 i}\left(x_{01}\right)}^{\gamma_{1 i}\left(\bar{x}_{1}\right)} \int_{\gamma_{2 i}\left(x_{02}\right)}^{\gamma_{2 i}\left(\bar{x}_{2}\right)} r_{i}\left(t_{1}, t_{2}\right) \Delta t_{2} \Delta t_{1} \\
& +\sum_{i=1}^{n} \int_{\gamma_{1 i}\left(x_{01}\right)}^{\gamma_{1 i}\left(x_{1}\right)} \int_{\gamma_{2 i}\left(x_{02}\right)}^{\gamma_{2 i}\left(x_{2}\right)} f_{i}\left(x_{1}, t_{1}, x_{2}, t_{2}\right) \\
& \times\left\{w_{2}\left(\sqrt[q_{1}]{\bar{\xi}_{1}\left(t_{1}, t_{2}\right)}\right)+\int_{\gamma_{1 i}\left(x_{01}\right)}^{t_{1}} \int_{\gamma_{2 i}\left(x_{02}\right)}^{t_{2}} g_{i}\left(t_{1}, m_{1}, t_{2}, m_{2}\right)\right. \\
& \left.\times w_{2}\left(\sqrt[q_{1}]{\bar{\xi}_{1}\left(m_{1}, m_{2}\right)}\right) \Delta m_{2} \Delta m_{1}\right\} \Delta t_{2} \Delta t_{1} \\
& =\bar{b}_{1}\left(\bar{x}_{1}, \bar{x}_{2}\right)+\sum_{i=1}^{n} \int_{\gamma_{1 i}\left(x_{01}\right)}^{\gamma_{1 i}\left(x_{1}\right)} \int_{\gamma_{2 i}\left(x_{02}\right)}^{\gamma_{2 i}\left(x_{2}\right)} f_{i}\left(x_{1}, t_{1}, x_{2}, t_{2}\right) \\
& \times\left\{w_{2}\left(\sqrt[q_{1}]{\bar{\xi}_{1}\left(t_{1}, t_{2}\right)}\right)+\int_{\gamma_{1 i}\left(x_{01}\right)}^{t_{1}} \int_{\gamma_{2 i}\left(x_{02}\right)}^{t_{2}} g_{i}\left(t_{1}, m_{1}, t_{2}, m_{2}\right)\right. \\
& \left.\times w_{2}\left(\sqrt[q_{1}]{\bar{\xi}_{1}\left(m_{1}, m_{2}\right)}\right) \Delta m_{2} \Delta m_{1}\right\} \Delta t_{2} \Delta t_{1}
\end{aligned}
$$

Let

$$
\begin{aligned}
\bar{\zeta}_{1}\left(x_{1}, x_{2}\right)= & \bar{b}_{1}\left(\bar{x}_{1}, \bar{x}_{2}\right)+\sum_{i=1}^{n} \int_{\gamma_{1 i}\left(x_{01}\right)}^{\gamma_{1 i}\left(x_{1}\right)} \int_{\gamma_{2 i}\left(x_{02}\right)}^{\gamma_{2 i}\left(x_{2}\right)} f_{i}\left(x_{1}, t_{1}, x_{2}, t_{2}\right) \\
& \times\left\{w_{2}\left(\sqrt[q_{1}]{\bar{\xi}_{1}\left(t_{1}, t_{2}\right)}\right)+\int_{\gamma_{1 i}\left(x_{01}\right)}^{t_{1}} \int_{\gamma_{2 i}\left(x_{02}\right)}^{t_{2}} g_{i}\left(t_{1}, m_{1}, t_{2}, m_{2}\right)\right. \\
& \left.\times w_{2}\left(\sqrt[q_{1}]{\bar{\xi}_{1}\left(m_{1}, m_{2}\right)}\right) \Delta m_{2} \Delta m_{1}\right\} \Delta t_{2} \Delta t_{1} .
\end{aligned}
$$

Then we have

$$
\bar{\zeta}_{1}\left(x_{01}, x_{2}\right)=\bar{\zeta}_{1}\left(x_{1}, x_{02}\right)=\bar{b}_{1}\left(\bar{x}_{1}, \bar{x}_{2}\right)
$$

and

$$
\bar{\xi}_{1}\left(x_{1}, x_{2}\right) \leq \bar{\zeta}_{1}^{\frac{q_{1}}{q_{1}-q_{2}}}\left(x_{1}, x_{2}\right)
$$


It follows from Lemma 2.1 and (3.44) that

$$
\begin{aligned}
\bar{\zeta}_{1}^{\Delta x_{1}}\left(x_{1}, x_{2}\right) \\
=\sum_{i=1}^{n} \gamma_{1 i}^{\Delta}\left(x_{1}\right) \int_{\gamma_{2 i}\left(x_{02}\right)}^{\gamma_{2 i}\left(x_{2}\right)} f_{i}\left(\sigma\left(x_{1}\right), \gamma_{1 i}\left(x_{1}\right), x_{2}, t_{2}\right)\left\{w_{2}\left(\sqrt[q_{1}]{\bar{\xi}_{1}\left(\gamma_{1 i}\left(x_{1}\right), t_{2}\right)}\right)\right. \\
\left.\quad+\int_{\gamma_{1 i}\left(x_{01}\right)}^{\gamma_{1 i}\left(x_{1}\right)} \int_{\gamma_{2 i}\left(x_{02}\right)}^{t_{2}} g_{i}\left(\gamma_{1 i}\left(x_{1}\right), m_{1}, t_{2}, m_{2}\right) \times w_{2}\left(\sqrt[q_{1}]{\bar{\xi}_{1}\left(m_{1}, m_{2}\right)}\right) \Delta m_{2} \Delta m_{1}\right\} \Delta t_{2} \\
+\sum_{i=1}^{n} \int_{\gamma_{1 i}\left(x_{01}\right)}^{\gamma_{1 i}\left(x_{1}\right)}\left[\int_{\gamma_{2 i}\left(x_{02}\right)}^{\gamma_{2 i}\left(x_{2}\right)} f_{i}\left(x_{1}, t_{1}, x_{2}, t_{2}\right) \times\left\{w_{2}\left(\sqrt[q_{1}]{\bar{\xi}_{1}\left(t_{1}, t_{2}\right)}\right)\right.\right. \\
\left.\left.\quad+\int_{\gamma_{1 i}\left(x_{01}\right)}^{t_{1}} \int_{\gamma_{2 i}\left(x_{02}\right)}^{t_{2}} g_{i}\left(t_{1}, m_{1}, t_{2}, m_{2}\right) w_{2}\left(\sqrt[q_{1}]{\bar{\xi}_{1}\left(m_{1}, m_{2}\right)}\right) \Delta m_{2} \Delta m_{1}\right\} \Delta t_{2}\right]^{\Delta x_{1}} \Delta t_{1} .
\end{aligned}
$$

It follows from (3.46) and the fact that $w_{2}$ and $\bar{\zeta}_{1}$ are nondecreasing that

$$
\begin{aligned}
& \frac{\bar{\zeta}_{1}^{\Delta x_{1}}\left(x_{1}, x_{2}\right)}{w_{2}\left(\sqrt[q_{1}-q_{2}]{\bar{\zeta}_{1}\left(x_{1}, x_{2}\right)}\right)} \\
& \leq \sum_{i=1}^{n}\left[\gamma_{1 i}^{\Delta}\left(x_{1}\right) \int_{\gamma_{2 i}\left(x_{02}\right)}^{\gamma_{2 i}\left(x_{2}\right)} f_{i}\left(\sigma\left(x_{1}\right), \gamma_{1 i}\left(x_{1}\right), x_{2}, t_{2}\right)\right. \\
& \quad \times\left\{1+\int_{\gamma_{1 i}\left(x_{01}\right)}^{\gamma_{1 i}\left(x_{1}\right)} \int_{\gamma_{2 i}\left(x_{02}\right)}^{t_{2}} g_{i}\left(\gamma_{1 i}\left(x_{1}\right), m_{1}, t_{2}, m_{2}\right) \Delta m_{2} \Delta m_{1}\right\} \Delta t_{2} \\
& \quad+\sum_{i=1}^{n} \int_{\gamma_{1 i}\left(x_{01}\right)}^{\gamma_{1 i}\left(x_{1}\right)}\left[\int_{\gamma_{2 i}\left(x_{02}\right)}^{\gamma_{2 i}\left(x_{2}\right)} f_{i}\left(x_{1}, t_{1}, x_{2}, t_{2}\right)\right. \\
& \left.\quad \times\left\{1+\int_{\gamma_{1 i}\left(x_{01}\right)}^{t_{1}} \int_{\gamma_{2 i}\left(x_{02}\right)}^{t_{2}} g_{i}\left(t_{1}, m_{1}, t_{2}, m_{2}\right) \Delta m_{2} \Delta m_{1}\right\} \Delta t_{2}\right]^{\Delta x_{1}} \Delta t_{1} \\
& =\sum_{i=1}^{n}\left[\int_{\gamma_{1 i}\left(x_{01}\right)}^{\gamma_{1 i}\left(x_{1}\right)} \int_{\gamma_{2 i}\left(x_{02}\right)}^{\gamma_{2 i}\left(x_{2}\right)} f_{i}\left(x_{1}, t_{1}, x_{2}, t_{2}\right)\right. \\
& \left.\quad \times\left\{1+\int_{\gamma_{1 i}\left(x_{01}\right)}^{t_{1}} \int_{\gamma_{2 i}\left(x_{02}\right)}^{t_{2}} g_{i}\left(t_{1}, m_{1}, t_{2}, m_{2}\right) \Delta m_{2} \Delta m_{1}\right\} \Delta t_{2} \Delta t_{1}\right]^{\Delta x_{1}} .
\end{aligned}
$$

Making use of (3.45) and the definition of $H_{1}$, integrating over $\left[x_{01}, x_{1}\right]$ gives

$$
\begin{aligned}
H_{1}\left(\bar{\zeta}_{1}\left(x_{1}, x_{2}\right)\right) \leq & H_{1}\left(\bar{b}_{1}\left(\bar{x}_{1}, x_{2}\right)\right)+\sum_{i=1}^{n} \int_{\gamma_{1 i}\left(x_{01}\right)}^{\gamma_{1 i}\left(x_{1}\right)} \int_{\gamma_{2 i}\left(x_{02}\right)}^{\gamma_{2 i}\left(x_{2}\right)} f_{i}\left(x_{1}, t_{1}, x_{2}, t_{2}\right) \\
& \times\left\{1+\int_{\gamma_{1 i}\left(x_{01}\right)}^{t_{1}} \int_{\gamma_{2 i}\left(x_{02}\right)}^{t_{2}} g_{i}\left(t_{1}, m_{1}, t_{2}, m_{2}\right) \Delta m_{2} \Delta m_{1}\right\} \Delta t_{2} \Delta t_{1} \\
= & H_{1}\left(\bar{b}_{1}\left(\bar{x}_{1}, x_{2}\right)\right)+c\left(x_{1}, x_{2}\right) .
\end{aligned}
$$

Combining (3.38), (3.46), and (3.47), we get

$$
u\left(x_{1}, x_{2}\right) \leq \sqrt[q_{1}-q_{2}]{H_{1}^{-1}\left(H_{1}\left(\bar{b}_{1}\left(\bar{x}_{1}, \bar{x}_{2}\right)\right)+c\left(x_{1}, x_{2}\right)\right)} .
$$


Letting $x_{1}=\bar{x}_{1}$ and $x_{2}=\bar{x}_{2}$ in (3.3), and considering $\bar{x}_{1} \in \mathbb{T}_{1}$ and $\bar{x}_{2} \in \mathbb{T}_{2}$ are arbitrary, after substituting $\bar{x}_{1}$ and $\bar{x}_{2}$ with $x_{1}$ and $x_{2}$, we obtain the desired result (3.35).

Let $\mathbb{T}=Z$. Then Theorem 3.1 leads to Corollary 3.13 immediately.

Corollary 3.13 Let $u, r_{i}, a_{j}: A_{1} \times A_{2} \rightarrow \mathbb{R}_{0}^{+}$and $f_{i}, g_{i}, f_{i}^{\Delta x_{1}}: A_{1}^{2} \times A_{2}^{2} \rightarrow \mathbb{R}_{0}^{+}$be nonnegative real-valued functions such that $a_{j}$ is nondecreasing with respect to its each variable, let $\gamma_{j i}: A_{j} \rightarrow \mathbb{R}_{0}^{+}$be a nonnegative and nondecreasing function, $\tilde{\mathfrak{a}}:\left\{-\rho_{1 i}, \ldots,-1,0\right\} \times$ $\left\{-\rho_{2 i}, \ldots,-1,0\right\} \rightarrow \mathbb{R}_{0}^{+}$be a nonnegative function, $-\infty<\tilde{\mathfrak{p}}_{j}=\inf \left\{\min \left(x_{j}-\rho_{j i}\right), x_{j} \in A_{j}\right\} \leq 0$, $w$ and $w_{j}$ be as defined in Theorem 3.1. If $u\left(x_{1}, x_{2}\right)$ satisfies the following discrete inequality

$$
\begin{aligned}
& w\left(u\left(x_{1}, x_{2}\right)\right) \\
& \leq a_{1}\left(x_{1}, x_{2}\right)+a_{2}\left(x_{1}, x_{2}\right) \sum_{i=1}^{n} \sum_{t_{1}=\gamma_{1 i}(0)}^{\gamma_{1 i}\left(x_{1}\right)-1} \sum_{t_{2}=\gamma_{2 i}(0)}^{\gamma_{2 i}\left(x_{2}\right)-1} w_{1}\left(u\left(t_{1}-\rho_{1 i}, t_{2}-\rho_{2 i}\right)\right) \\
& \quad \times\left[f _ { i } ( x _ { 1 } , t _ { 1 } , x _ { 2 } , t _ { 2 } ) \left\{w_{2}\left(u\left(t_{1}-\rho_{1 i}, t_{2}-\rho_{2 i}\right)\right)+\prod_{l=1}^{2} \sum_{m_{l}=\gamma_{l i}(0)}^{t_{l}-1} g_{i}\left(t_{1}, m_{1}, t_{2}, m_{2}\right)\right.\right. \\
& \left.\left.\quad \times w_{2}\left(u\left(m_{1}-\rho_{1 i}, m_{2}-\rho_{2 i}\right)\right)\right\}+r_{i}\left(t_{1}, t_{2}\right)\right]
\end{aligned}
$$

with the following initial condition

$$
\left\{\begin{array}{l}
w\left(u\left(x_{1}, x_{2}\right)\right)=\tilde{\mathfrak{a}}\left(x_{1}, x_{2}\right), \quad x_{1} \in\left[\tilde{\mathfrak{p}}_{1}, 0\right] \text { or } x_{2} \in\left[\tilde{\mathfrak{p}}_{2}, 0\right] \\
\tilde{\mathfrak{a}}\left(x_{1}-\rho_{1 i}, x_{2}-\rho_{2 i}\right) \leq a_{1}\left(x_{1}, x_{2}\right), \quad x_{1} \leq \rho_{1 i}, \text { or } x_{2} \leq \rho_{2 i}
\end{array}\right.
$$

then

$$
u\left(x_{1}, x_{2}\right) \leq w^{-1}\left(H_{2}^{-1}\left(H_{3}^{-1}\left(H_{3}\left(\tilde{b}_{1}\left(x_{1}, x_{2}\right)\right)+a_{2}\left(x_{1}, x_{2}\right) \tilde{c}\left(x_{1}, x_{2}\right)\right)\right)\right)
$$

for all $0 \leq x_{1} \leq \tilde{x}_{1}$ and $0 \leq x_{2} \leq \tilde{x}_{2}$, where

$$
\begin{aligned}
& \begin{aligned}
\tilde{c}\left(x_{1}, x_{2}\right)= & \sum_{i=1}^{n} \sum_{t_{1}=\gamma_{1 i}(0)}^{\gamma_{1 i}\left(x_{1}\right)-1} \sum_{t_{2}=\gamma_{2 i}(0)}^{\gamma_{2 i}\left(x_{2}\right)-1} f_{i}\left(x_{1}, t_{1}, x_{2}, t_{2}\right) \\
& \times\left(1+\sum_{m_{1}=\gamma_{1 i}(0)}^{t_{1}-1} \sum_{m_{2}=\gamma_{2 i}(0)}^{t_{2}-1} g_{i}\left(t_{1}, m_{1}, t_{2}, m_{2}\right)\right)
\end{aligned} \\
& \tilde{b}_{1}\left(x_{1}, x_{2}\right)=H_{2}\left(a_{1}\left(x_{1}, x_{2}\right)\right)+a_{2}\left(x_{1}, x_{2}\right) \sum_{i=1}^{n} \sum_{t_{1}=\gamma_{1 i}(0)}^{\gamma_{1 i}\left(x_{1}\right)-1} \sum_{t_{2}=\gamma_{2 i}(0)}^{\gamma_{2 i}\left(x_{2}\right)-1} r_{i}\left(t_{1}, t_{2}\right), \\
& H_{2}(s)=\int_{s_{7}}^{s} \frac{d p}{w_{1}\left(w^{-1}(p)\right)}, s>s_{7}>0 \text { with } H_{2}(\infty)=\infty, \\
& H_{3}(s)=\int_{s_{8}}^{s} \frac{d p}{w_{2}\left(w^{-1}\left(H_{2}^{-1}(p)\right)\right)}, \quad s>s_{8}>0 \text { with } H_{3}(\infty)=\infty,
\end{aligned}
$$


$H_{2}^{-1}$ and $H_{3}^{-1}$ are respectively the inverse functions of $H_{2}$ and $H_{3}$, and $\tilde{x}_{1}$ and $\tilde{x}_{2}$ are chosen such that

$$
\begin{aligned}
& H_{3}\left(\tilde{b}_{1}\left(x_{1}, x_{2}\right)\right)+a_{2}\left(x_{1}, x_{2}\right) \tilde{c}\left(x_{1}, x_{2}\right) \in \operatorname{Dom}\left(H_{3}^{-1}\right), \\
& H_{3}^{-1}\left(H_{3}\left(\tilde{b}_{1}\left(x_{1}, x_{2}\right)\right)+a_{2}\left(x_{1}, x_{2}\right) \tilde{c}\left(x_{1}, x_{2}\right)\right) \in \operatorname{Dom}\left(H_{2}^{-1}\right), \\
& H_{2}^{-1}\left(H_{3}^{-1}\left(H_{3}\left(\tilde{b}_{1}\left(x_{1}, x_{2}\right)\right)+a_{2}\left(x_{1}, x_{2}\right) \tilde{c}\left(x_{1}, x_{2}\right)\right)\right) \in \operatorname{Dom}\left(w^{-1}\right) .
\end{aligned}
$$

\section{Applications}

Example 4.1 Consider the following integro-differential equation with several arguments:

$$
\begin{aligned}
{\left[u\left(x_{1}, x_{2}\right)\right]^{\Delta x_{1} \Delta x_{2}}=} & \mathrm{F}\left[x_{1}, t_{1}, x_{2}, t_{2}, u\left(\mu_{11}\left(t_{1}\right), \mu_{21}\left(t_{2}\right)\right), \ldots, u\left(\mu_{1 n}\left(t_{1}\right), \mu_{2 n}\left(t_{2}\right)\right),\right. \\
& \int_{x_{01}}^{t_{1}} \int_{x_{02}}^{t_{2}} \mathbf{Q}\left(t_{1}, m_{1}, t_{2}, m_{2}, u\left(\mu_{11}\left(m_{1}\right), \mu_{21}\left(m_{2}\right)\right),\right. \\
& \left.\left.\ldots, u\left(\mu_{1 n}\left(m_{1}\right), \mu_{2 n}\left(m_{2}\right)\right)\right) \Delta m_{2} \Delta m_{1}\right]
\end{aligned}
$$

with the initial condition

$$
\left\{\begin{array}{l}
{\left[u\left(x_{1}, x_{02}\right)\right]^{\Delta x_{2}}=\mathfrak{a}_{1}^{\Delta}\left(x_{1}\right), \quad u\left(x_{01}, x_{2}\right)=\mathfrak{a}_{2}\left(x_{2}\right),} \\
u\left(x_{1}, x_{2}\right)=\mathfrak{a}\left(x_{1}, x_{2}\right), \quad x_{1} \in\left[\mathfrak{p}_{1}, x_{01}\right]_{\mathbb{T}} \text { or } x_{2} \in\left[\mathfrak{p}_{2}, x_{02}\right]_{\mathbb{T}}, \\
\left|\mathfrak{a}\left(\mu_{1 i}\left(x_{1}\right), \mu_{2 i}\left(x_{2}\right)\right)\right| \leq\left|a_{1}\left(x_{1}, x_{2}\right)\right|, \quad \mu_{1 i}\left(x_{1}\right) \leq x_{01} \text { or } \mu_{2 i}\left(x_{2}\right) \leq x_{02}
\end{array}\right.
$$

for $\mathrm{F}: \mathbb{T}_{1}^{2} \times \mathbb{T}_{2}^{2} \times \mathrm{R}^{n+1} \rightarrow \mathbb{R}$ is right-dense continuous on $\mathbb{T}_{1}^{2} \times \mathbb{T}_{2}^{2}$ and continuous on $\mathbb{R}^{n+1}$, $\mathbf{Q}: \mathbb{T}_{1}^{2} \times \mathbb{T}_{2}^{2} \times \mathbb{R}^{n} \rightarrow \mathbb{R}$ is right-dense continuous on $\mathbb{T}_{1}^{2} \times \mathbb{T}_{2}^{2}$ and continuous on $\mathbb{R}^{n}, u$ : $\mathbb{T}_{1} \times \mathbb{T}_{2} \rightarrow \mathbb{R} /\{0\}, \mathfrak{a}_{j}: \mathbb{T}_{j} \rightarrow \mathbb{R}, \mathfrak{a}:\left(\left[\mathfrak{p}_{1}, x_{01}\right] \times\left[\mathfrak{p}_{2}, x_{02}\right]\right)_{\mathbb{T}^{2}} \rightarrow \mathbb{R}, a_{1}: \mathbb{T}_{1} \times \mathbb{T}_{2} \rightarrow \mathbb{R}$ are rightdense continuous functions and $\mu_{j i}$ is as defined in Theorem 3.1.

Theorem 4.2 Assume that

$$
\left.\begin{array}{rl}
\left|\mathrm{F}\left(x_{1}, t_{1}, x_{2}, t_{2}, \mathfrak{k}_{1}, \mathfrak{k}_{2}, \ldots, \mathfrak{k}_{n}, k\right)\right| \leq & a_{2}\left(x_{1}, x_{2}\right) \sum_{i=1}^{n} w_{1}\left(\left|\mathfrak{k}_{i}\right|\right) \\
& \times\left[f_{i}\left(x_{1}, t_{1}, x_{2}, t_{2}\right)\left\{w_{2}\left(\left|\mathfrak{k}_{i}\right|\right)+|k|\right\}+r_{i}\left(t_{1}, t_{2}\right)\right], \\
\left|\mathbf{Q}\left(x_{1}, t_{1}, x_{2}, t_{2}, \mathfrak{k}_{1}, \ldots, \mathfrak{k}_{n}\right)\right| \leq & g_{i}\left(x_{1}, t_{1}, x_{2}, t_{2}\right) w_{2}\left(\left|\mathfrak{k}_{i}\right|\right),
\end{array}\right\}
$$

where $f_{i}, g_{i}, r_{i}, a_{2}$ are as defined in Theorem 3.1, $a_{1}\left(x_{1}, x_{2}\right)=\sum_{j=1}^{2} \mathfrak{a}_{j}\left(x_{j}\right), w_{1}(\eta)=\sqrt[3]{\sigma^{2}(\eta)}+$ $\sqrt[3]{\sigma(\eta) \eta}+\sqrt[3]{\eta^{2}}, w_{2}(\eta)=\sqrt{\sigma(\sqrt[3]{\eta})}+\sqrt[6]{\eta}$ for $\eta \in \mathbb{R}_{0}^{+}$, and $u\left(x_{1}, x_{2}\right)$ is a solution of equation (4.1) with initial condition (4.2), then

$$
\left|u\left(x_{1}, x_{2}\right)\right| \leq\left(\sqrt{\mathfrak{b}_{3}\left(x_{1}, x_{2}\right)}+a_{2}\left(x_{1}, x_{2}\right) \mathfrak{b}_{4}\left(x_{1}, x_{2}\right)\right)^{6},
$$

where

$$
\mathfrak{b}_{3}\left(x_{1}, x_{2}\right)=\sqrt[3]{\left|a_{1}\left(x_{1}, x_{2}\right)\right|}+a_{2}\left(x_{1}, x_{2}\right) \sum_{i=1}^{n} \int_{x_{01}}^{x_{1}} \int_{x_{02}}^{x_{2}} r_{i}\left(t_{1}, t_{2}\right) \Delta t_{2} \Delta t_{1}
$$




$$
\begin{aligned}
\mathfrak{b}_{4}\left(x_{1}, x_{2}\right)= & \sum_{i=1}^{n} \int_{x_{01}}^{x_{1}} \int_{x_{02}}^{x_{2}} f_{i}\left(x_{1}, t_{1}, x_{2}, t_{2}\right) \\
& \times\left(1+\int_{x_{01}}^{t_{1}} \int_{x_{02}}^{t_{2}} g_{i}\left(t_{1}, m_{1}, t_{2}, m_{2}\right) \Delta m_{2} \Delta m_{1}\right) \Delta t_{2} \Delta t_{1} .
\end{aligned}
$$

Proof Let $\tilde{\mathbb{T}}=\varrho\left(\mathbb{T}_{1}, x_{2}\right)$ and $\tilde{G}_{j}(\eta)=\sqrt[4-j]{\eta}$, where $\varrho\left(x_{1}, x_{2}\right)$ is strictly increasing with respect to $x_{1} \in \mathbb{T}_{1}$. Then it follows from Theorem 2.3 that

$$
\begin{aligned}
{\left[\tilde{G}_{1}\left(\varrho\left(x_{1}, x_{2}\right)\right)\right]^{\Delta x_{1}} } & =\tilde{G}_{1}^{\tilde{\Delta}}(\varrho) \varrho^{\Delta x_{1}}\left(x_{1}, x_{2}\right) \\
& =\frac{\varrho^{\Delta x_{1}}\left(x_{1}, x_{2}\right)}{\sqrt[3]{\sigma^{2}\left(\varrho\left(x_{1}, x_{2}\right)\right)}+\sqrt[3]{\sigma\left(\varrho\left(x_{1}, x_{2}\right)\right) \varrho\left(x_{1}, x_{2}\right)}+\sqrt[3]{\varrho^{2}\left(x_{1}, x_{2}\right)}} \\
& =\frac{\varrho^{\Delta x_{1}}\left(x_{1}, x_{2}\right)}{w_{1}\left(\varrho\left(x_{1}, x_{2}\right)\right)}=\frac{\varrho^{\Delta x_{1}}\left(x_{1}, x_{2}\right)}{w_{1}\left(w^{-1}\left(\varrho\left(x_{1}, x_{2}\right)\right)\right)}, \\
{\left[\tilde{G}_{2}\left(\varrho\left(x_{1}, x_{2}\right)\right)\right]^{\Delta x_{1}} } & =\tilde{G}_{2}^{\tilde{\Delta}}(\varrho) \varrho^{\Delta x_{1}}\left(x_{1}, x_{2}\right) \\
& =\frac{\varrho^{\Delta x_{1}}\left(x_{1}, x_{2}\right)}{\sqrt{\left.\sigma\left(x_{1}, x_{2}\right)\right)}+\sqrt{\varrho\left(x_{1}, x_{2}\right)}} \\
& =\frac{\varrho^{\Delta x_{1}}\left(x_{1}, x_{2}\right)}{w_{2}\left(\varrho^{3}\left(x_{1}, x_{2}\right)\right)}=\frac{\varrho^{\Delta x_{1}}\left(x_{1}, x_{2}\right)}{w_{2}\left(w^{-1}\left(\tilde{G}_{1}^{-1}\left(\varrho\left(x_{1}, x_{2}\right)\right)\right)\right)} .
\end{aligned}
$$

From (4.1) and (4.2) we get

$$
\begin{aligned}
u\left(x_{1}, x_{2}\right)= & a_{1}\left(x_{1}, x_{2}\right)+\int_{x_{01}}^{x_{1}} \int_{x_{02}}^{x_{2}} \mathrm{~F}\left[x_{1}, t_{1}, x_{2}, t_{2}, u\left(\mu_{11}\left(t_{1}\right), \mu_{21}\left(t_{2}\right)\right),\right. \\
& \ldots, u\left(\mu_{1 n}\left(t_{1}\right), \mu_{2 n}\left(t_{2}\right)\right), \int_{x_{01}}^{t_{1}} \int_{x_{02}}^{t_{2}} \mathbf{Q}\left(t_{1}, m_{1}, t_{2}, m_{2}, u\left(\mu_{11}\left(m_{1}\right), \mu_{21}\left(m_{2}\right)\right),\right. \\
& \left.\left.\ldots, u\left(\mu_{1 n}\left(m_{1}\right), \mu_{2 n}\left(m_{2}\right)\right)\right) \Delta m_{2} \Delta m_{1}\right] \Delta t_{2} \Delta t_{1} .
\end{aligned}
$$

From (4.3) we know that (4.5) has the form

$$
\begin{aligned}
\left|u\left(x_{1}, x_{2}\right)\right| \leq & \left|a_{1}\left(x_{1}, x_{2}\right)\right|+\int_{x_{01}}^{x_{1}} \int_{x_{02}}^{x_{2}} \mid \mathrm{F}\left[x_{1}, t_{1}, x_{2}, t_{2}, u\left(\mu_{11}\left(t_{1}\right), \mu_{21}\left(t_{2}\right)\right),\right. \\
& \ldots, u\left(\mu_{1 n}\left(t_{1}\right), \mu_{2 n}\left(t_{2}\right)\right), \int_{x_{01}}^{t_{1}} \int_{x_{02}}^{t_{2}} \mathbf{Q}\left(t_{1}, m_{1}, t_{2}, m_{2}, u\left(\mu_{11}\left(m_{1}\right), \mu_{21}\left(m_{2}\right)\right),\right. \\
& \left.\left.\ldots, u\left(\mu_{1 n}\left(m_{1}\right), \mu_{2 n}\left(m_{2}\right)\right)\right) \Delta m_{2} \Delta m_{1}\right] \mid \Delta t_{2} \Delta t_{1} \\
\leq & \left|a_{1}\left(x_{1}, x_{2}\right)\right|+a_{2}\left(x_{1}, x_{2}\right) \sum_{i=1}^{n} \int_{x_{01}}^{x_{1}} \int_{x_{02}}^{x_{2}} w_{1}\left(\left|u\left(\mu_{1 i}\left(t_{1}\right), \mu_{2 i}\left(t_{2}\right)\right)\right|\right) \\
& \times\left[f _ { i } ( x _ { 1 } , t _ { 1 } , x _ { 2 } , t _ { 2 } ) \left\{w_{2}\left(\left|u\left(\mu_{1 i}\left(t_{1}\right), \mu_{2 i}\left(t_{2}\right)\right)\right|\right)+\int_{x_{01}}^{t_{1}} \int_{x_{02}}^{t_{2}} g_{i}\left(t_{1}, m_{1}, t_{2}, m_{2}\right)\right.\right. \\
& \left.\left.\times w_{2}\left(\left|u\left(\mu_{1 i}\left(m_{1}\right), \mu_{2 i}\left(m_{2}\right)\right)\right|\right) \Delta m_{2} \Delta m_{1}\right\}+r_{i}\left(t_{1}, t_{2}\right)\right] \Delta t_{2} \Delta t_{1} .
\end{aligned}
$$

Therefore, the desired result follows easily from (3.3) and (4.6). 
Example 4.3 Consider another integro-differential equation with several arguments:

$$
\begin{aligned}
& {\left[u^{\frac{2}{5}}\left(x_{1}, x_{2}\right) u^{\Delta x_{1}}\left(x_{1}, x_{2}\right)\right]^{\Delta x_{2}} \mathrm{~F}\left[x_{1}, t_{1}, x_{2}, t_{2}, u\left(\mu_{11}\left(t_{1}\right), \mu_{21}\left(t_{2}\right)\right),\right.} \\
& \quad \ldots, u\left(\mu_{1 n}\left(t_{1}\right), \mu_{2 n}\left(t_{2}\right)\right), \int_{x_{01}}^{t_{1}} \int_{x_{02}}^{t_{2}} \mathbf{Q}\left(t_{1}, m_{1}, t_{2}, m_{2}, u\left(\mu_{11}\left(m_{1}\right), \mu_{21}\left(m_{2}\right)\right),\right. \\
& \left.\left.\quad \ldots, u\left(\mu_{1 n}\left(m_{1}\right), \mu_{2 n}\left(m_{2}\right)\right)\right) \Delta m_{2} \Delta m_{1}\right]
\end{aligned}
$$

with the initial condition

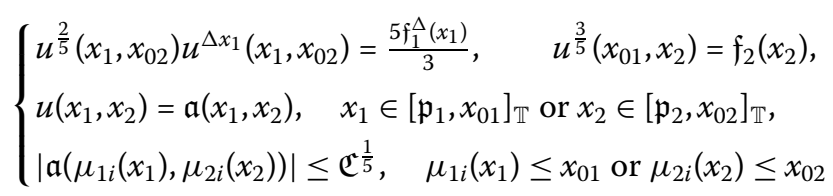

for $\mathrm{F}: \mathbb{T}_{1}^{2} \times \mathbb{T}_{2}^{2} \times \mathbb{R}^{n+1} \rightarrow \mathbb{R}$ is right-dense continuous on $\mathbb{T}_{1}^{2} \times \mathbb{T}_{2}^{2}$ and continuous on $\mathbb{R}^{n+1}, \mathbf{Q}: \mathbb{T}_{1}^{2} \times \mathbb{T}_{2}^{2} \times \mathbb{R}^{n} \rightarrow \mathbb{R}$ is right-dense continuous on $\mathbb{T}_{1}^{2} \times \mathbb{T}_{2}^{2}$ and continuous on $\mathbb{R}^{n}$, $u: \mathbb{T}_{1} \times \mathbb{T}_{2} \rightarrow \mathbb{R} /\{0\}, \mathfrak{f}_{j}: \mathbb{T}_{j} \rightarrow \mathbb{R}, \mathfrak{a}:\left(\left[\mathfrak{p}_{1}, x_{01}\right] \times\left[\mathfrak{p}_{2}, x_{02}\right]\right)_{\mathbb{T}^{2}} \rightarrow \mathbb{R}$ are right-dense continuous functions, $\mathfrak{C}$ is a nonzero constant such that $\mathfrak{C} \geq \sum_{j=1}^{2}\left|\mathfrak{f}_{j}\left(x_{j}\right)\right|$ and $\mu_{j i}$ is as defined in Theorem 3.1.

\section{Theorem 4.4 Assume that}

$$
\left.\begin{array}{c}
\left|\mathrm{F}\left(x_{1}, t_{1}, x_{2}, t_{2}, \mathfrak{k}_{1}, \mathfrak{k}_{2}, \ldots, \mathfrak{k}_{n}, k\right)\right| \\
\leq \sum_{i=1}^{n} \frac{\left|\mathfrak{k}_{i}\right|^{2}}{3}\left[f_{i}\left(x_{1}, t_{1}, x_{2}, t_{2}\right)\left\{w_{2}\left(\left|\mathfrak{k}_{i}\right|\right)+|k|\right\}+r_{i}\left(t_{1}, t_{2}\right)\right], \\
\left|\mathrm{Q}\left(x_{1}, t_{1}, x_{2}, t_{2}, \mathfrak{k}_{1}, \ldots, \mathfrak{k}_{n}\right)\right| \leq g_{i}\left(x_{1}, t_{1}, x_{2}, t_{2}\right) w_{2}\left(\left|\mathfrak{k}_{i}\right|\right),
\end{array}\right\}
$$

where $f_{i}, g_{i}$, and $r_{i}$ are as defined in Theorem 3.1, $w_{2}(\eta)=\sqrt[3]{\sigma^{2}\left(\eta^{3}\right)}+\sqrt[3]{\sigma\left(\eta^{3}\right) \eta^{3}}+\eta^{2}$ for $\eta \in \mathbb{R}_{0}^{+}$. If $u\left(x_{1}, x_{2}\right)$ is a solution of equation (4.7) satisfying initial condition (4.8), then

$$
\left|u\left(x_{1}, x_{2}\right)\right| \leq \sqrt[3]{\overline{\mathfrak{b}}_{3}\left(x_{1}, x_{2}\right)}+\mathfrak{b}_{4}\left(x_{1}, x_{2}\right)
$$

where $\mathfrak{b}_{4}\left(x_{1}, x_{2}\right)$ is defined by (4.4) and

$$
\overline{\mathfrak{b}}_{3}\left(x_{1}, x_{2}\right):=\mathfrak{C}^{\frac{3}{5}}+\sum_{i=1}^{n} \int_{x_{01}}^{x_{1}} \int_{x_{02}}^{x_{2}} r_{i}\left(t_{1}, t_{2}\right) \Delta t_{2} \Delta t_{1} .
$$

Proof Let $\overline{\mathbb{T}}=\varpi\left(\mathbb{T}_{1}, x_{2}\right)$ and $\bar{G}_{2}(\eta)=\sqrt[3]{\eta}$, where $\varpi$ is strictly increasing with respect to $x_{1} \in \mathbb{T}_{1}$. Then by Theorem 2.3 we have

$$
\begin{aligned}
{\left[\bar{G}_{2}\left(\varpi\left(x_{1}, x_{2}\right)\right)\right]^{\Delta x_{1}} } & =\bar{G}_{2}^{\bar{\Delta}}(\varpi) \varpi^{\Delta x_{1}}\left(x_{1}, x_{2}\right) \\
& =\frac{\varpi^{\Delta x_{1}}\left(x_{1}, x_{2}\right)}{\sqrt[3]{\sigma^{2}\left(\varpi\left(x_{1}, x_{2}\right)\right)}+\sqrt[3]{\sigma\left(\varpi\left(x_{1}, x_{2}\right)\right) \varpi\left(x_{1}, x_{2}\right)}+\sqrt[3]{\varpi^{2}\left(x_{1}, x_{2}\right)}} \\
& =\frac{\varpi^{\Delta x_{1}\left(x_{1}, x_{2}\right)}}{w_{2}\left(\sqrt[3]{\varpi\left(x_{1}, x_{2}\right)}\right)} .
\end{aligned}
$$


Integrating equation (4.7) over $\left[x_{02}, x_{2}\right]$ gives

$$
\begin{aligned}
u^{\frac{2}{5}}\left(x_{1}, x_{2}\right) u^{\Delta x_{1}}\left(x_{1}, x_{2}\right) & \\
= & u^{\frac{2}{5}}\left(x_{1}, x_{02}\right) u^{\Delta x_{1}}\left(x_{1}, x_{02}\right)+\int_{x_{02}}^{x_{2}} \mathrm{~F}\left[x_{1}, t_{1}, x_{2}, t_{2}, u\left(\mu_{11}\left(t_{1}\right), \mu_{21}\left(t_{2}\right)\right), \ldots,\right. \\
& u\left(\mu_{1 n}\left(t_{1}\right), \mu_{2 n}\left(t_{2}\right)\right), \int_{x_{01}}^{t_{1}} \int_{x_{02}}^{t_{2}} \mathbf{Q}\left(t_{1}, m_{1}, t_{2}, m_{2}, u\left(\mu_{11}\left(m_{1}\right), \mu_{21}\left(m_{2}\right)\right), \ldots,\right. \\
& \left.\left.u\left(\mu_{1 n}\left(m_{1}\right), \mu_{2 n}\left(m_{2}\right)\right)\right) \Delta m_{2} \Delta m_{1}\right] \Delta t_{2} .
\end{aligned}
$$

It follows from Theorem 2.2 and $\frac{u^{\Delta x_{1}}\left(x_{1}, x_{2}\right)}{u\left(x_{1}, x_{2}\right)} \geq 0$ that

$$
\begin{aligned}
\left(\frac{5}{3} u^{\frac{3}{5}}\left(x_{1}, x_{2}\right)\right)^{\Delta x_{1}} & =u^{\Delta x_{1}}\left(x_{1}, x_{2}\right) \int_{0}^{1}\left\{u\left(x_{1}, x_{2}\right)+h \mu\left(x_{1}, x_{2}\right) u^{\Delta x_{1}}\left(x_{1}, x_{2}\right)\right\}^{-\frac{2}{5}} d h \\
& =\frac{u^{\Delta x_{1}}\left(x_{1}, x_{2}\right)}{u^{\frac{2}{5}}\left(x_{1}, x_{2}\right)} \int_{0}^{1}\left\{1+h \mu\left(x_{1}, x_{2}\right) \frac{u^{\Delta x_{1}}\left(x_{1}, x_{2}\right)}{u\left(x_{1}, x_{2}\right)}\right\}^{-\frac{2}{5}} d h \\
& =\frac{u^{\Delta x_{1}}\left(x_{1}, x_{2}\right)}{u^{\frac{2}{5}}\left(x_{1}, x_{2}\right)} \times \frac{5\left\{1+\mu\left(x_{1}, x_{2}\right) \frac{u^{\Delta x_{1}\left(\mathfrak{x}_{1}, \mathfrak{x}_{2}\right)}}{u\left(x_{1}, x_{2}\right)}\right\}^{\frac{3}{5}}-5}{3 \mu\left(x_{1}, x_{2}\right) \frac{u^{\Delta x_{1}\left(x_{1}, x_{2}\right)}}{u\left(x_{1}, x_{2}\right)}} \\
& \leq \frac{u^{\Delta x_{1}}\left(x_{1}, x_{2}\right)}{u^{\frac{2}{5}}\left(x_{1}, x_{2}\right)} .
\end{aligned}
$$

From (4.10) and (4.11) one has

$$
\begin{aligned}
& \left(\frac{5}{3} u^{\frac{3}{5}}\left(x_{1}, x_{2}\right)\right)^{\Delta x_{1}} \\
& \leq \frac{5 \mathfrak{f}_{1}^{\Delta}\left(x_{1}\right)}{3}+\int_{x_{02}}^{x_{2}} \mathrm{~F}\left[x_{1}, t_{1}, x_{2}, t_{2}, u\left(\mu_{11}\left(t_{1}\right), \mu_{21}\left(t_{2}\right)\right),\right. \\
& \quad \ldots, u\left(\mu_{1 n}\left(t_{1}\right), \mu_{2 n}\left(t_{2}\right)\right), \int_{x_{01}}^{t_{1}} \int_{x_{02}}^{t_{2}} \mathbf{Q}\left(t_{1}, m_{1}, t_{2}, m_{2}, u\left(\mu_{11}\left(m_{1}\right), \mu_{21}\left(m_{2}\right)\right), \ldots,\right. \\
& \left.\left.\quad u\left(\mu_{1 n}\left(m_{1}\right), \mu_{2 n}\left(m_{2}\right)\right)\right) \Delta m_{2} \Delta m_{1}\right] \Delta t_{2} .
\end{aligned}
$$

Integrating over $\left[\mathfrak{x}_{01}, \mathfrak{x}_{1}\right]$ leads to

$$
\begin{aligned}
u^{\frac{3}{5}}\left(x_{1}, x_{2}\right) \leq & \mathfrak{f}_{1}\left(x_{1}\right)+\mathfrak{f}_{2}\left(x_{2}\right)+\frac{3}{5} \int_{x_{01}}^{x_{1}} \int_{x_{02}}^{x_{2}} \mathrm{~F}\left[x_{1}, t_{1}, x_{2}, t_{2}, u\left(\mu_{11}\left(t_{1}\right), \mu_{21}\left(t_{2}\right)\right)\right. \\
& \ldots, u\left(\mu_{1 n}\left(t_{1}\right), \mu_{2 n}\left(t_{2}\right)\right), \int_{x_{01}}^{t_{1}} \int_{x_{02}}^{t_{2}} \mathbf{Q}\left(t_{1}, m_{1}, t_{2}, m_{2}, u\left(\mu_{11}\left(m_{1}\right), \mu_{21}\left(m_{2}\right)\right)\right. \\
& \left.\left.\ldots, u\left(\mu_{1 n}\left(m_{1}\right), \mu_{2 n}\left(m_{2}\right)\right)\right) \Delta m_{2} \Delta m_{1}\right] \Delta t_{2} \Delta t_{1} .
\end{aligned}
$$


Table 1 The value of $u(x ; y)$ from (4.8) and (4.10)

\begin{tabular}{lll}
\hline$(x, y)$ & $(4.8)$ & $(4.10)$ \\
\hline$(2,2)$ & $3.0692 \mathrm{e}+11$ & $5.7300 \mathrm{e}+11$ \\
$(2,5)$ & $3.1140 \mathrm{e}+11$ & $1.9384 \mathrm{e}+12$ \\
$(2,9)$ & $3.1481 \mathrm{e}+11$ & $9.2602 \mathrm{e}+12$ \\
$(3,4)$ & $3.1193 \mathrm{e}+11$ & $2.8268 \mathrm{e}+12$ \\
$(3,8)$ & $3.1605 \mathrm{e}+11$ & $3.9592 \mathrm{e}+13$ \\
$(7,3)$ & $3.1497 \mathrm{e}+11$ & $1.7927 \mathrm{e}+13$ \\
$(11,5)$ & $3.2673 \mathrm{e}+26$ & $6.3683 \mathrm{e}+26$ \\
$(15,2)$ & $3.1803 \mathrm{e}+11$ & $1.2336 \mathrm{e}+14$ \\
$(40,1)$ & $3.2160 \mathrm{e}+11$ & $1.9787 \mathrm{e}+14$ \\
\hline
\end{tabular}

From (4.9) we know that inequality (4.12) has the form

$$
\begin{aligned}
\left|u^{\frac{3}{5}}\left(x_{1}, x_{2}\right)\right| \leq & \mathfrak{C}+\frac{3}{5} \int_{x_{01}}^{x_{1}} \int_{x_{02}}^{x_{2}} \mid \mathrm{F}\left[x_{1}, t_{1}, x_{2}, t_{2}, u\left(\mu_{11}\left(t_{1}\right), \mu_{21}\left(t_{2}\right)\right)\right. \\
& \ldots, u\left(\mu_{1 n}\left(t_{1}\right), \mu_{2 n}\left(t_{2}\right)\right), \int_{x_{01}}^{t_{1}} \int_{x_{02}}^{t_{2}} \mathbf{Q}\left(t_{1}, m_{1}, t_{2}, m_{2}, u\left(\mu_{11}\left(m_{1}\right), \mu_{21}\left(m_{2}\right)\right)\right. \\
& \left.\left.\ldots, u\left(\mu_{1 n}\left(m_{1}\right), \mu_{2 n}\left(m_{2}\right)\right)\right) \Delta m_{2} \Delta m_{1}\right] \mid \Delta t_{2} \Delta t_{1} \\
\leq & \mathfrak{C}+\frac{1}{5} \sum_{i=1}^{n} \int_{x_{01}}^{x_{1}} \int_{x_{02}}^{x_{2}}\left|u\left(\mu_{1 i}\left(t_{1}\right), \mu_{2 i}\left(t_{2}\right)\right)\right|^{2}\left[f_{i}\left(x_{1}, t_{1}, x_{2}, t_{2}\right)\right. \\
& \times\left\{w_{2}\left(\left|u\left(\mu_{1 i}\left(t_{1}\right), \mu_{2 i}\left(t_{2}\right)\right)\right|\right)+\int_{x_{01}}^{t_{1}} \int_{x_{02}}^{t_{2}} g_{i}\left(t_{1}, m_{1}, t_{2}, m_{2}\right)\right. \\
& \left.\left.\times w_{2}\left(\left|u\left(\mu_{1 i}\left(m_{1}\right), \mu_{2 i}\left(m_{2}\right)\right)\right|\right) \Delta m_{2} \Delta m_{1}\right\}+r_{i}\left(t_{1}, t_{2}\right)\right] \Delta t_{2} \Delta t_{1}
\end{aligned}
$$

Therefore, the desired result follows from (3.5) and (4.13).

Example 4.5 Consider the delay discrete inequality (4.8) satisfying initial condition (4.9) with $u\left(x_{1}, x_{2}\right)=27^{x_{1} x_{2}}, \rho_{j i}=j i, a_{1}\left(x_{1}, x_{2}\right)=27^{8}, a_{2}\left(x_{1}, x_{2}\right)=\sqrt[10]{x_{1} x_{2}}, f_{i}\left(x_{1}, t_{1}, x_{2}, t_{2}\right)=$ $\arctan \left(\sqrt[i+1]{x_{1}+t_{1}+x_{2}+t_{2}}\right), r_{i}\left(x_{1}, x_{2}\right)=\sqrt[i+1]{\exp \left(x_{1} x_{2}\right)}, \gamma_{j i}=w=I, g_{i}\left(x_{1}, t_{1}, x_{2}, t_{2}\right)=10^{-i-1} \times$ $\sqrt[i+1]{x_{1}+t_{1}+x_{2}+t_{2}}(1 \leq i \leq 2)$, and $w_{j}$ is defined as in Theorem 4.2.

We find that the numerical solution agrees with the analytical solution for some discrete inequalities by calculating the value of $u(x, y)$ from (4.8) and (4.10) (see Table 1).

\section{Conclusion}

In the article, we have presented several explicit bounds for the delay double integral inequalities on time scales and have given their applications to the solutions of certain integro-differential equations. Our results are the improvements and generalizations of some previously known results. Furthermore, we have found some new estimates for the integral inequalities in the form of exponential function.

\section{Acknowledgements}

The authors would like to express their sincere thanks to the editor and the anonymous reviewers for their helpful comments and suggestions.

\section{Funding}

The work was supported by the Natural Science Foundation of China (Grant Nos. 61673169, 11301127, 11701176 $11626101,11601485)$ 
Availability of data and materials

Not applicable.

\section{Competing interests}

The authors declare that they have no competing interests.

\section{Authors' contributions}

All authors contributed equally to the writing of this paper. All authors read and approved the final manuscript.

\section{Author details}

'Department of Mathematics and Statistics, University of Lahore, Lahore, Pakistan. ${ }^{2}$ School of Mathematical Sciences, Zhejiang University, Hangzhou, China. ${ }^{3}$ Department of Mathematics, University of Engineering and Technology, Lahore, Pakistan. ${ }^{4}$ Department of Mathematics, Government College (GC) University, Faisalabad, Pakistan. ${ }^{5}$ Department of Mathematics, Huzhou University, Huzhou, China.

\section{Publisher's Note}

Springer Nature remains neutral with regard to jurisdictional claims in published maps and institutional affiliations.

Received: 15 October 2019 Accepted: 13 January 2020 Published online: 20 January 2020

\section{References}

1. Hilger, S.: Analysis on measure chains-a unified approach to continuous and discrete calculus. Results Math. 18(1-2), 18-56 (1990)

2. Huang, C.-X., Liu, L.-Z.: Sharp function inequalities and boundedness for Toeplitz type operator related to general fractional singular integral operator. Publ. Inst. Math. 92(106), 165-176 (2012)

3. Li, J., Guo, B.-L.: The quasi-reversibility method to solve the Cauchy problems for parabolic equations. Acta Math. Sin. 29(8), 1617-1628 (2013)

4. Huang, C.-X., Guo, S., Liu, L.-Z.: Boundedness on Morrey space for Toeplitz type operator associated to singular integral operator with variable Calderón-Zygmund kernel. J. Math. Inequal. 8(3), 453-464 (2014)

5. Huang, C.-X., Yang, Z.-C., Yi, T.-S., Zou, X.-F.: On the basins of attraction for a class of delay differential equations with non-monotone bistable nonlinearities. J. Differ. Equ. 256(7), 2101-2114 (2014)

6. Deng, Y.-J., Fang, X.-P., Li, J.: Numerical methods for reconstruction of the source term of heat equations from the final overdetermination. Bull. Korean Math. Soc. 52(5), 1495-1515 (2015)

7. Fang, X.-P., Deng, Y.-J., Li, J.: Plasmon resonance and heat generation in nanostructures. Math. Methods Appl. Sci. 38(18), 4663-4672 (2015)

8. Xie, Y.-Q., Li, Q.-S., Zhu, K.-X.: Attractors for nonclassical diffusion equations with arbitrary polynomial growth nonlinearity. Nonlinear Anal., Real World Appl. 31, 23-37 (2016)

9. Tan, Y.-X., Liu, L.-Z.: Weighted boundedness of multilinear operator associated to singular integral operator with variable Calderón-Zygmund kernel. Rev. R. Acad. Cienc. Exactas Fís. Nat., Ser. A Mat. 111(4), $931-946$ (2017)

10. Cai, Z.-W., Huang, J.-H., Huang, L.-H.: Generalized Lyapunov-Razumikhin method for retarded differential inclusions: applications to discontinuous neural networks. Discrete Contin. Dyn. Syst., Ser. B 22(9), 3591-3614 (2017)

11. Duan, L., Huang, L.-H., Guo, Z.-Y., Fang, X.-W.: Periodic attractor for reaction-diffusion high-order Hopfield neural networks with time-varying delays. Comput. Math. Appl. 73(2), 233-245 (2017)

12. Huang, C.-X., Liu, L.-Z.: Boundedness of multilinear singular integral operator with a non-smooth kernel and mean oscillation. Quaest. Math. 40(3), 295-312 (2017)

13. Duan, L., Huang, C.-X.: Existence and global attractivity of almost periodic solutions for a delayed differential neoclassical growth model. Math. Methods Appl. Sci. 40(3), 814-822 (2017)

14. Hu, H.-J., Liu, L.-Z.: Weighted inequalities for a general commutator associated to a singular integral operator satisfying a variant of Hörmander's condition. Math. Notes 101(5-6), 830-840 (2017)

15. Hu, H.-J., Zou, X.-F.: Existence of an extinction wave in the Fisher equation with a shifting habitat. Proc. Am. Math. Soc. 145(11), 4763-4771 (2017)

16. Cai, Z.-W., Huang, J.-H., Huang, L.-H.: Periodic orbit analysis for the delayed Filippov system. Proc. Am. Math. Soc. 146(11), 4667-4682 (2018)

17. Chen, T., Huang, L.-H., Yu, P., Huang, W.-T.: Bifurcation of limit cycles at infinity in piecewise polynomial systems. Nonlinear Anal., Real World Appl. 41, 82-106 (2018)

18. Duan, L., Fang, X.-W., Huang, C.-X.: Global exponential convergence in a delayed almost periodic Nicholson's blowflies model with discontinuous harvesting. Math. Methods Appl. Sci. 41(5), 1954-1965 (2018)

19. Tan, Y.-X., Huang, C.-X., Sun, B., Wang, T.: Dynamics of a class of delayed reaction-diffusion systems with Neumann boundary condition. J. Math. Anal. Appl. 458(2), 1115-1130 (2018)

20. Wang, J.-F., Chen, X.-Y., Huang, L.-H.: The number and stability of limit cycles for planar piecewise linear systems of node-saddle type. J. Math. Anal. Appl. 469(1), 405-427 (2019)

21. Wang, J.-F., Huang, C.-X., Huang, L.-H.: Discontinuity-induced limit cycles in a general planar piecewise linear system of saddle-focus type. Nonlinear Anal. Hybrid Syst. 33, 162-178 (2019)

22. Huang, C.-X., Zhang, H., Huang, L.-H.: Almost periodicity analysis for a delayed Nicholson's blowflies model with nonlinear density-dependent mortality term. Commun. Pure Appl. Anal. 18(6), 3337-3349 (2019)

23. Chu, Y.-M., Wang, M.-K., Qiu, S.-L.: Optimal combinations bounds of root-square and arithmetic means for Toader mean. Proc. Indian Acad. Sci. Math. Sci. 122(1), 41-51 (2012)

24. Xu, H.-Z., Chu, Y.-M., Qian, W.-M.: Sharp bounds for the Sándor-Yang means in terms of arithmetic and contra-harmonic means. J. Inequal. Appl. 2018, Article ID 127 (2018)

25. Wu, S.-H., Chu, Y.-M.: Schur m-power convexity of generalized geometric Bonferroni mean involving three parameters. J. Inequal. Appl. 2019, Article ID 57 (2019) 
26. Qian, W.-M., Xu, H.-Z., Chu, Y.-M.: Improvements of bounds for the Sándor-Yang means. J. Inequal. Appl. 2019, Article ID 73 (2019)

27. He, X.-H., Qian, W.-M., Xu, H.-Z., Chu, Y.-M.: Sharp power mean bounds for two Sándor-Yang means. Rev. R. Acad. Cienc. Exactas Fís. Nat., Ser. A Mat. 113(3), 2627-2638 (2019)

28. Qian, W.-M., He, Z.-Y., Zhang, H.-W., Chu, Y.-M.: Sharp bounds for Neumann means in terms of two-parameter contraharmonic and arithmetic mean. J. Inequal. Appl. 2019, Article ID 168 (2019)

29. Qian, W.-M., Yang, Y.-Y., Zhang, H.-W., Chu, Y.-M.: Optimal two-parameter geometric and arithmetic mean bounds for the Sándor-Yang mean. J. Inequal. Appl. 2019, Article ID 287 (2019)

30. Qian, W.-M., Zhang, W., Chu, Y.-M.: Bounding the convex combination of arithmetic and integral means in terms of one-parameter harmonic and geometric means. Miskolc Math. Notes 20(2), 1157-1166 (2019)

31. Wang, B., Luo, C.-L., Li, S.-H., Chu, Y.-M.: Sharp one-parameter geometric and quadratic means bounds for the Sándor-Yang means. Rev. R. Acad. Cienc. Exactas Fís. Nat., Ser. A Mat. 114(2), Article ID 7 (2019). https://doi.org/10.1007/s13398-019-00734-0

32. Dai, Z.-F., Wen, F.-H.: Another improved Wei-Yao-Liu nonlinear conjugate gradient method with sufficient descent property. Appl. Math. Comput. 218(14), 7421-7430 (2012)

33. Li, X.-F., Tang, G.-J., Tang, B.-Q.: Stress field around a strike-slip fault in orthotropic elastic layers via a hypersingular integral equation. Comput. Math. Appl. 66(11), 2317-2326 (2013)

34. Zhou, W.-J., Chen, X.-L.: On the convergence of a modified regularized Newton method for convex optimization with singular solutions. J. Comput. Appl. Math. 239, 179-188 (2013)

35. Wang, W.-S.: Stability of solutions of nonlinear neutral differential equations with piecewise constant delay and their discretizations. Appl. Math. Comput. 219(9), 4590-4600 (2013)

36. Zhao, K., Yao, G.-Z.: Application of the alternating direction method for an inverse monic quadratic eigenvalue problem. Appl. Math. Comput. 244, 32-41 (2014)

37. Wang, W.-S., Fang, Q., Zhuang, Y., Li, S.-F.: Asymptotic stability of solution to nonlinear neutral and Volterra functional differential equations in Banach spaces. Appl. Math. Comput. 237, 217-226 (2014)

38. Tang, W.-S., Sun, Y.-J.: Construction of Runge-Kutta type methods for solving ordinary differential equations. Appl. Math. Comput. 234, 179-191 (2014)

39. Zhao, K., Liao, A.-P., Yao, G.-Z.: A proximal point-like method for symmetric finite element model updating problems. Comput. Appl. Math. 34(3), 1251-1268 (2015)

40. Xie, D.-X., Li, J.: A new analysis of electrostatic free energy minimization and Poisson-Boltzmann equation for protein in ionic solvent. Nonlinear Anal., Real World Appl. 21, 185-196 (2015)

41. Dai, Z.-F., Chen, X.-H., Wen, F.-H.: A modified Perry's conjugate gradient method-based derivative-free method for solving large-scale nonlinear monotone equations. Appl. Math. Comput. 270, 378-386 (2015)

42. Feng, L.-B., Zhuang, P., Liu, F., Turner, I., Anh, V., Li, J.: A fast second-order accurate method for a two-sided space-fractional diffusion equation with variable coefficients. Comput. Math. Appl. 73(6), 1155-1171 (2017)

43. Li, J., Liu, F., Fang, L., Turner, I.: A novel finite volume method for the Riesz space distributed-order diffusion equation. Comput. Math. Appl. 74(4), 772-783 (2017)

44. Wang, W.-S.: Fully-geometric mesh one-leg methods for the generalized pantograph equation: approximating Lyapunov functional and asymptotic contractivity. Appl. Numer. Math. 117, 50-68 (2017)

45. Dong, X.-L., Han, D.-R., Ghanbari, R., Li, X.-L., Dai, Z.-F.: Some new three-term Hestenes-Stiefel conjugate gradient methods with affine combination. Optimization 66(5), 759-776 (2017)

46. Liu, Z.-Y., Wu, N.-C., Qin, X.-R., Zhang, Y.-L.: Trigonometric transform splitting methods for real symmetric Toeplitz systems. Comput. Math. Appl. 75(8), 2782-2794 (2018)

47. Liu, S., Chen, Y.-P., Huang, Y.-Q., Zhou, J.: An efficient two grid method for miscible displacement problem approximated by mixed finite element methods. Comput. Math. Appl. 77(3), 752-764 (2019)

48. Tang, W.-S., Zhang, J.-J.: Symmetric integrators based on continuous-stage Runge-Kutta-Nyström methods for reversible systems. Appl. Math. Comput. 361, 1-12 (2019)

49. Li, J., Ying, J.-Y., Xie, D.-X.: On the analysis and application of an ion size-modified Poisson-Boltzmann equation. Nonlinear Anal., Real World Appl. 47, 188-203 (2019)

50. Zhao, T.-H., Chu, Y.-M., Wang, H.: Logarithmically complete monotonicity properties relating to the gamma function. Abstr. Appl. Anal. 2011, Article ID 896483 (2011)

51. Chu, Y.-M., Adil Khan, M., Ali, T., Dragomir, S.S.: Inequalities for $\alpha$-fractional differentiable functions. J. Inequal. Appl. 2017, Article ID 93 (2017)

52. Huang, T.-R., Han, B.-W., Ma, X.-Y., Chu, Y.-M.: Optimal bounds for the generalized Euler-Mascheroni constant. J. Inequal. Appl. 2018, Article ID 118 (2018)

53. Song, Y.-Q., Adil Khan, M., Zaheer Ullah, S., Chu, Y.-M.: Integral inequalities involving strongly convex functions. J. Funct. Spaces 2018, Article ID 6595921 (2018)

54. Adil Khan, M., Iqbal, A., Suleman, M., Chu, Y.-M.: Hermite-Hadamard type inequalities for fractional integrals via Green's function. J. Inequal. Appl. 2018, Article ID 161 (2018)

55. Adil Khan, M., Khurshid, Y., Du, T.-S., Chu, Y.-M.: Generalization of Hermite-Hadamard type inequalities via conformable fractional integrals. J. Funct. Spaces 2018, Article ID 5357463 (2018)

56. Huang, T.-R., Tan, S.-Y., Ma, X.-Y., Chu, Y.-M.: Monotonicity properties and bounds for the complete $p$-elliptic integrals. J. Inequal. Appl. 2018, Article ID 239 (2018)

57. Zaheer Ullah, S., Adil Khan, M., Chu, Y.-M.: Majorization theorems for strongly convex functions. J. Inequal. Appl. 2019, Article ID 58 (2019)

58. Wang, M.-K., Chu, Y.-M., Zhang, W.: Monotonicity and inequalities involving zero-balanced hypergeometric function. Math. Inequal. Appl. 22(2), 601-617 (2019)

59. Zaheer Ullah, S., Adil Khan, M., Khan, Z.A., Chu, Y.-M.: Integral majorization type inequalities for the functions in the sense of strong convexity. J. Funct. Spaces 2019, Article ID 9487823 (2019)

60. Adil Khan, M., Zaheer Ullah, S., Chu, Y.-M.: The concept of coordinate strongly convex functions and related inequalities. Rev. R. Acad. Cienc. Exactas Fís. Nat., Ser. A Mat. 113(3), 2235-2251 (2019)

61. Adil Khan, M., Hanif, M., Khan, Z.A., Ahmad, K., Chu, Y.-M.: Association of Jensen's inequality for s-convex function with Csiszár divergence. J. Inequal. Appl. 2019, Article ID 162 (2019) 
62. Wang, M.-K., Zhang, W., Chu, Y.-M.: Monotonicity, convexity and inequalities involving the generalized elliptic integrals. Acta Math. Sci. Ser. B Engl. Ed. 39(5), 1440-1450 (2019)

63. Latif, M.A., Rashid, S., Dragomir, S.S., Chu, Y.-M.: Hermite-Hadamard type inequalities for co-ordinated convex and quasi-convex functions and their applications. J. Inequal. Appl. 2019, Article ID 317 (2019)

64. Wang, M.-K., Chu, H.-H., Chu, Y.-M.: Precise bounds for the weighted Hölder mean of the complete $p$-elliptic integrals. J. Math. Anal. Appl. 480(2), Article ID 123388 (2009). https://doi.org/10.1016/j.jmaa.2019.123388

65. Zaheer Ullah, S., Adil Khan, M., Chu, Y.-M.: A note on generalized convex functions. J. Inequal. Appl. 2019, Article ID $291(2019)$

66. Abbas Baloch, I., Chu, Y.-M.: Petrović-type inequalities for harmonic $h$-convex functions. J. Funct. Spaces 2020, Article ID 3075390 (2020)

67. Khan, S., Adil Khan, M., Chu, Y.-M.: Converse of the Jensen inequality derived from the Green functions with applications in information theory. Math. Methods Appl. Sci. (2019). https://doi.org/10.1002/mma.6066

68. Qian, W.-M., He, Z.-Y., Chu, Y.-M.: Approximation for the complete elliptic integral of the first kind. Rev. R. Acad. Cienc. Exactas Fís. Nat., Ser. A Mat. (2020). https://doi.org/10.1007/s13398-020-00784-9

69. Wang, M.-K., He, Z.-Y., Chu, Y.-M.: Sharp power mean inequalities for the generalized elliptic integral of the first kind. Comput. Methods Funct. Theory. https://doi.org/10.1007/s40315-020-00298-w

70. Wang, H.-S., Gao, Z.-Q., Liu, Q.-S.: Central limit theorems for a supercritical branching process in a random environment. Stat. Probab. Lett. 81(5), 539-547 (2011)

71. Jin, Q.-Y., Grama, I., Liu, Q.-S.: A new Poisson noise filter based on weights optimization. J. Sci. Comput. 58(3), $548-573(2014)$

72. Luo, X.-Q., Du, Q.-K., Liu, L.-B.: A D-N alternating algorithm for exterior 3-D Poisson problem with prolate spheroid boundary. Appl. Math. Comput. 269, 252-264 (2015)

73. Liu, W., Ma, C.-Q., Li, Y.-Q., Wang, S.-M.: A strong limit theorem for the average of ternary functions of Markov chains in bi-infinite random environments. Stat. Probab. Lett. 100, 12-18 (2015)

74. Gao, Z.-Q., Liu, Q.-S.: First- and second-order expansions in the central limit theorem for a branching random walk. C. R. Math. Acad. Sci. Paris 354(5), 532-537 (2016)

75. Li, Y.-Q., Liu, Q.-S., Peng, X.-L.: Harmonic moments, large and moderate deviation principles for Mandelbrot's cascade in a random environment. Stat. Probab. Lett. 147, 57-65 (2019)

76. Agarwal, R.P., Ahmad, B., Alsaedi, A., Shahzad, N.: Dimension of the solution set for fractional differential inclusions. J. Nonlinear Convex Anal. 14(2), 319-329 (2013)

77. Baleanu, D., Mohammadi, H., Rezapour, S.: On a nonlinear fractional differential equation on partially ordered metric spaces. Adv. Differ. Equ. 2013, Article ID 83 (2013)

78. Baleanu, D., Rezapour, S., Mohammadi, H.: Some existence results on nonlinear fractional differential equations. Philos. Trans. R. Soc. Lond. A, Math. Phys. Eng. Sci. 371(1990), Article ID 20120144 (2013)

79. Baleanu, D., Mohammadi, H., Rezapour, S.: The existence of solutions for a nonlinear mixed problem of singular fractional differential equations. Adv. Differ. Equ. 2013, Article ID 359 (2013)

80. Baleanu, D., Rezapour, S., Etemad, S., Alsaedi, A.: On a time-fractional integrodifferential equation via three-point boundary value conditions. Math. Probl. Eng. 2015, Article ID 785738 (2015)

81. Baleanu, D., Khan, H., Jafari, H., Khan, R.A., Alipour, M.: On existence results for solutions of a coupled system of hybrid boundary value problems with hybrid conditions. Adv. Differ. Equ. 2015, Article ID 318 (2015)

82. Baleanu, D., Hedayati, V., Rezapour, S., Al Qurashi, M.: On two fractional differential inclusions. SpringerPlus 5, Article ID 882 (2016)

83. Ruzhansky, M., Cho, Y.J., Agarwal, P., Area, l.: Advances in Real and Complex Analysis with Applications. Springer, Singapore (2017)

84. Baleanu, D., Mousalou, A., Rezapour, S.: A new method for investigating approximate solutions of some fractional integro-differential equations involving the Caputo-Fabrizio derivative. Adv. Differ. Equ. 2017, Article ID 51 (2017)

85. Baleanu, D., Mousalou, A., Rezapour, S.: On the existence of solutions for some infinite coefficient-symmetric Caputo-Fabrizio fractional integro-differential equations. Bound. Value Probl. 2017, Article ID 145 (2017)

86. Aydogan, S.M., Baleanu, D., Mousalou, A., Rezapour, S.: On approximate solutions for two higher-order Caputo-Fabrizio fractional integro-differential equations. Adv. Differ. Equ. 2017, Article ID 221 (2017)

87. Agarwal, P.: Some inequalities involving Hadamard-type k-fractional integral operators. Math. Methods Appl. Sci. 40(11), 3882-3891 (2017)

88. Aydogan, M.S., Baleanu, D., Mousalou, A., Rezapour, S.: On high order fractional integro-differential equations including the Caputo-Fabrizio derivative. Bound. Value Probl. 2018, Article ID 90 (2018)

89. Baleanu, D., Ghafarnezhad, K., Rezapour, S., Shabibi, M.: On the existence of solutions of a three steps crisis integro-differential equation. Adv. Differ. Equ. 2018, Article ID 135 (2018)

90. Sitho, S., Ntouyas, S.K., Agarwal, P., Tariboon, J.: Noninstantaneous impulsive inequalities via conformable fractional calculus. J. Inequal. Appl. 2018, Article ID 261 (2018)

91. Baleanu, D., Mousalou, A., Rezapour, S.: The extended fractional Caputo-Fabrizio derivative of order $0 \leq \sigma<1$ on $C_{\mathbb{R}}[0,1]$ and the existence of solutions for two higher-order series-type differential equations. Adv. Differ. Equ. 2018, Article ID 255 (2018)

92. Baleanu, D., Rezapour, S., Saberpour, Z.: On fractional integro-differential inclusions via the extended fractional Caputo-Fabrizio derivation. Bound. Value Probl. 2019, Article ID 79 (2019)

93. Mehrez, K., Agarwal, P.: New Hermite-Hadamard type integral inequalities for convex functions and their applications. J. Comput. Appl. Math. 350, 274-285 (2019)

94. Baleanu, D., Ghafarnezhad, K., Rezapour, S.: On a three step crisis integro-differential equation. Adv. Differ. Equ. 2019, Article ID 153 (2019)

95. Jain, S., Agarwal, P.: On new applications of fractional calculus. Bol. Soc. Parana. Mat. (3) 37(3), 113-118 (2019)

96. El-Sayed, A.A., Agarwal, P.: Numerical solution of multiterm variable-order fractional differential equations via shifted Legendre polynomials. Math. Methods Appl. Sci. 42(11), 3978-3991 (2019)

97. Baleanu, D., Etemad, S., Pourrazi, S., Rezapour, S.: On the new fractional hybrid boundary value problems with three-point integral hybrid conditions. Adv. Differ. Equ. 2019, Article ID 473 (2019) 
98. Tomar, M., Agarwal, P., Choi, J.: Hermite-Hadamard type inequalities for generalized convex functions on fractal sets style. Bol. Soc. Parana. Mat. (3) 38(1), 101-106 (2020)

99. El-Deeb, A.A., El-Sennary, H.A., Agarwal, P.: Some Opial-type inequalities with higher order delta derivatives on time scales. Rev. R. Acad. Cienc. Exactas Fís. Nat., Ser. A Mat. 114(1), Article ID 29 (2020). https://doi.org/10.1007/s13398-019-00749-7

100. Rezapour, S., Saberpour, Z:: On dimension of the set of solutions of a fractional differential inclusion via the Caputo-Hadamard fractional derivation. J. Math. Ext. 14(1) (2020). Available online at https://ijmex.com/index.php/ijmex/article/view/1058

101. Gronwall, T.H.: Note on the derivatives with respect to a parameter of the solutions of a system of differential equations. Ann. Math. (2) 20(4), 292-296 (1919)

102. Bellman, R.: The stability of solutions of linear differential equations. Duke Math. J. 10, 643-647 (1943)

103. Li, W.-N.: Some delay Gronwall type inequalities on time scales. Acta Math. Appl. Sin. 31(4), 1103-1114 (2015)

104. Mi, Y.-Z: A generalized Gronwall-Bellman type delay integral inequality with two independent variables on time scales. J. Math. Inequal. 11(4), 1151-1160 (2017)

105. El-Deeb, A.A., Xu, H.-Y., Abdeldaim, A., Wang, G.-T.: Some dynamic inequalities on time scales and their applications. Adv. Differ. Equ. 2019, Article ID 130 (2019)

106. Khan, Z.A.: On some explicit bounds of integral inequalities related to time scales. Adv. Differ. Equ. 2019, Article ID $243(2019)$

107. El-Deeb, A.A., Cheung, W.-S.: A variety of dynamic inequalities on time scales with retardation. J. Nonlinear Sci. Appl. $11(10), 1185-1206(2018)$

108. Bohner, M., Peterson, A.: Dynamic Equations on Time Scales. Birkhäuser Boston, Boston (2001)

109. Cheung, W.-S., Ma, Q.-H., Pečarić, J.: Some discrete nonlinear inequalities and applications to difference equations. Acta Math. Sci. Ser. B Engl. Ed. 28(2), 417-430 (2008)

110. Cheung, W.-S., Ren, J.-L.: Discrete non-linear inequalities and applications to boundary value problems. J. Math. Anal. Appl. 319(2), 708-724 (2006)

111. Cho, Y.J., Kim, Y.-H., Pečarić, J.: New Gronwall-Ou-lang type integral inequalities and their applications. ANZIAM J. 50(1), 111-127 (2008)

112. Kim, Y.-H.: Gronwall, Bellman and Pachpatte type integral inequalities with applications. Nonlinear Anal. 71(12), e2641-e2656 (2009)

113. Feng, Q.-H., Fu, B.-S.: Generalized delay integral inequalities in two independent variables on time scales. WSEAS Trans. Math. 12(7), 757-766 (2013)

114. Feng, Q.-H., Meng, F.-W., Zhang, Y.-M.: Generalized Gronwall-Bellman-type discrete inequalities and their applications. J. Inequal. Appl. 2011, Article ID 47 (2011)

115. Wang, W.-S.: Some generalized nonlinear retarded integral inequalities with applications. J. Inequal. Appl. 2012, Article ID 31 (2012)

\section{Submit your manuscript to a SpringerOpen ${ }^{\circ}$ journal and benefit from:}

- Convenient online submission

- Rigorous peer review

- Open access: articles freely available online

- High visibility within the field

- Retaining the copyright to your article

Submit your next manuscript at $\boldsymbol{\nabla}$ springeropen.com 